\title{
Electrochemical TEMPO-Catalyzed Oxidative Ugi-type Reaction
}

Na Pan, $\dagger+$ Maegan Xinen Lee, $\uparrow$ Louis Bunel, $\uparrow$ Laurence Grimaud $\dagger *$ and Maxime R. Vitale $\dagger^{*}$

† Laboratoire des biomolécules, LBM, Département de chimie, École Normale Supérieure, PSL University, Sorbonne Université, 75005 Paris, France

\$ Shanghai Engineering Research Center of Molecular Therapeutics and New Drug Development, SCME, East China

Normal University, 3663 Zhongshanbei Road, 200062, Shanghai, China.

\section{Supporting Information}

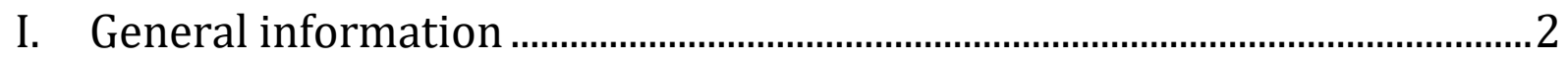

II. Condition screening....................................................................................

III. General procedure for electrosynthesis ………..............................................

IV. Characterization of all multicomponent adducts.......................................

V. Gram scale reaction ................................................................................... 34

VI. NMR Spectra of all multicomponent adducts..............................................35 


\section{General information}

Unless otherwise stated, commercially available materials were used as received. Reactions were monitored by thin-layer chromatography (TLC) analysis using precoated silica gel aluminium foils (with fluorescent indicator $254 \mathrm{~nm}$ ). Spots were visualized by UV irradiation or Kagi stain. Column chromatography was performed on silica gel (230-400 mesh, 40-63 $\mu \mathrm{m})$.

${ }^{1} \mathrm{H}(300 \mathrm{MHz}),{ }^{13} \mathrm{C}\left\{{ }^{1} \mathrm{H}\right\}(75 \mathrm{MHz})$ and ${ }^{19} \mathrm{~F}\left\{{ }^{1} \mathrm{H}\right\}(282 \mathrm{MHz})$ NMR spectra were recorded on a Bruker spectrometer. HRMS Spectra were recorded on a JEOL JMS-GC Mate II apparatus.

Electrosynthesis was performed under constant current with indicated current density after the indicated reaction time with IKA ElectraSyn 2.0 in a $5 \mathrm{~mL}$ cell equipped with a graphite plate as anode and a nickel plate as cathode.

\section{Condition screening}

1,3,5-Trimethoxybenzene, an internal standard for ${ }^{1} \mathrm{H}$ NMR, was added into each reaction of condition screening after the indicated reaction time. The NMR yields were calculated according to the exact amount of the added standard. 
a) Solvent

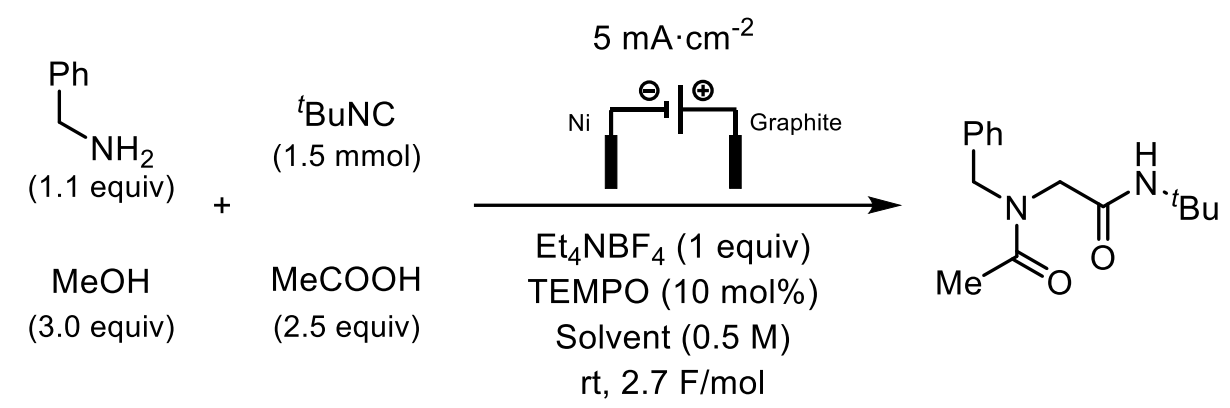

\begin{tabular}{|l|l|l|}
\hline Entry & Solvent & NMR yield (\%) \\
\hline 1 & $\mathrm{CH}_{3} \mathrm{CN}$ & 68 \\
\hline 2 & DCM & 75 \\
\hline 3 & DCE $^{*}$ & 7 \\
\hline
\end{tabular}

* $10 \%$ of $\mathrm{H}_{2} \mathrm{O}$ was added to dissolve all the substrates.

b) Concentration

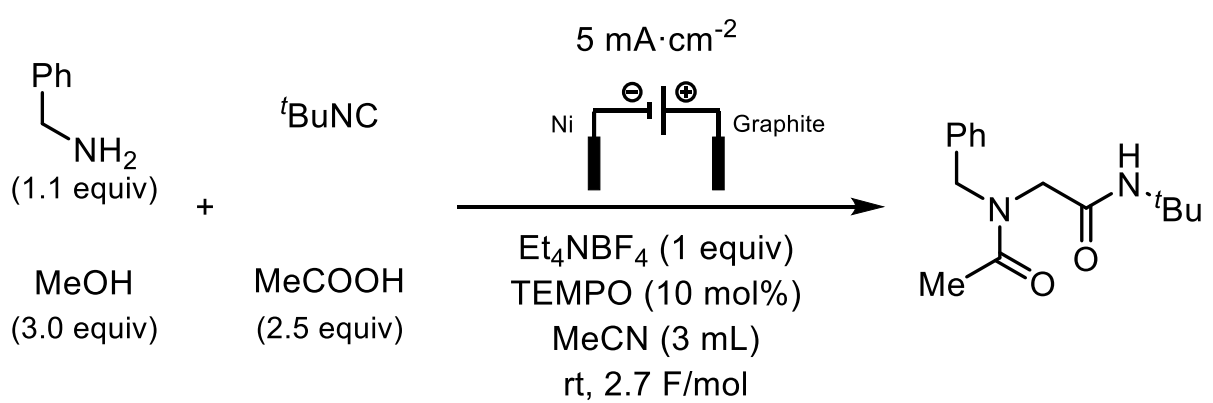

\begin{tabular}{|l|l|l|l|}
\hline Entry & Concentration $(\mathrm{mM})$ & Time $(\mathrm{h})$ & NMR yield (\%) \\
\hline 1 & 0.50 & 7.5 & 68 \\
\hline 4 & 0.17 & 2.5 & 39 \\
\hline
\end{tabular}

c) Cathode

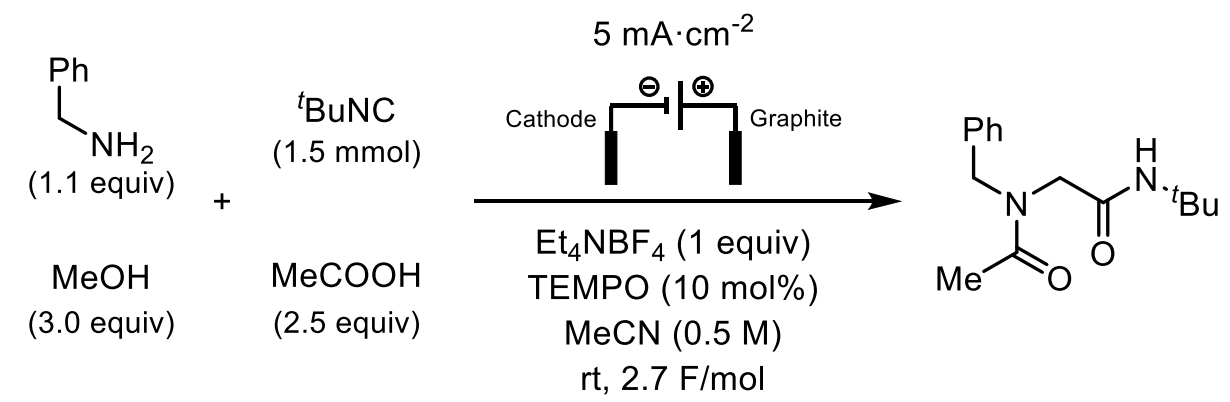




\begin{tabular}{|l|l|l|}
\hline Entry & Cathode & NMR yield (\%) \\
\hline 1 & Ni & 68 \\
\hline 5 & Ni foam & 69 \\
\hline 6 & Stainless steel & 68 \\
\hline
\end{tabular}

d) Anode

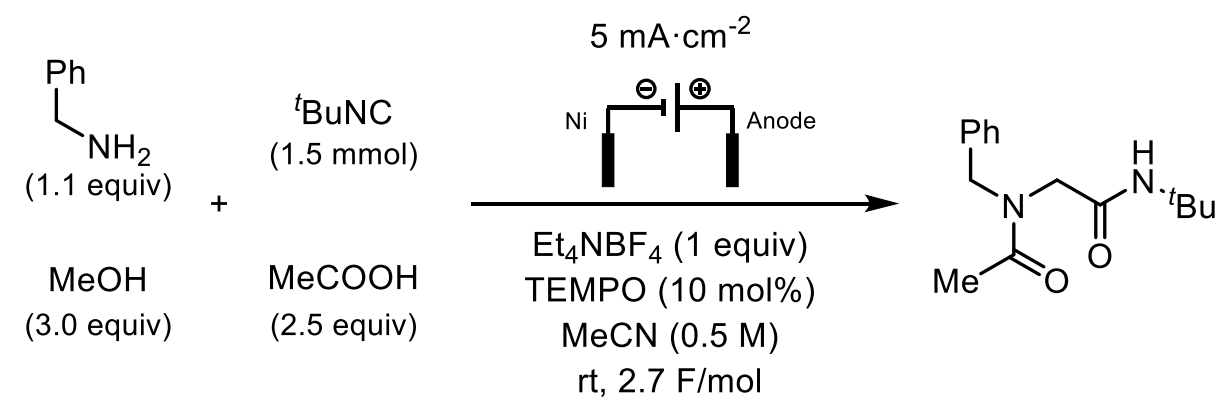

\begin{tabular}{|l|l|l|}
\hline Entry & Anode & NMR yield (\%) \\
\hline 1 & Graphite & 68 \\
\hline 7 & Ni & 54 \\
\hline 8 & Stainless steel & 53 \\
\hline
\end{tabular}

e) Acid

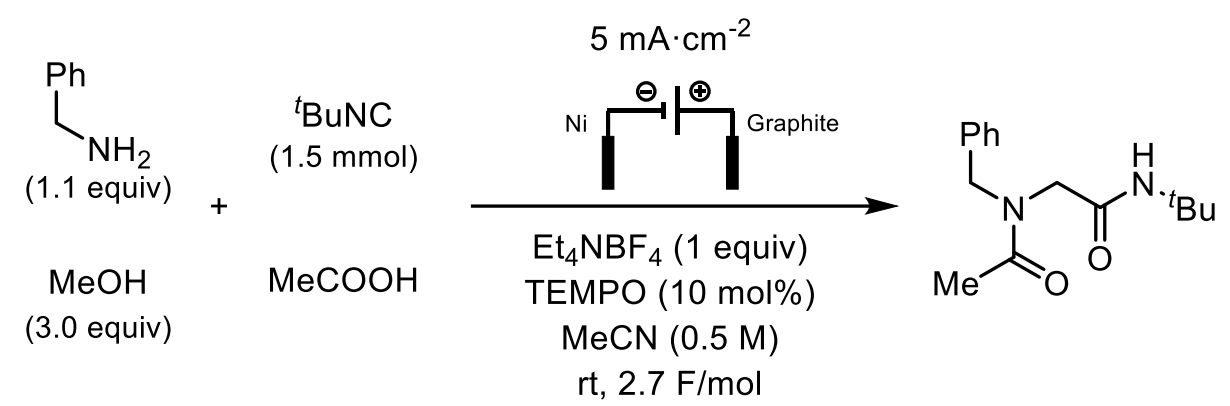

\begin{tabular}{|l|l|l|}
\hline Entry & Acid & NMR yield (\%) \\
\hline 1 & 2.5 equiv & 68 \\
\hline 9 & 1.5 equiv & 57 \\
\hline 10 & 3.5 equiv & 69 \\
\hline
\end{tabular}


f) Alcohol

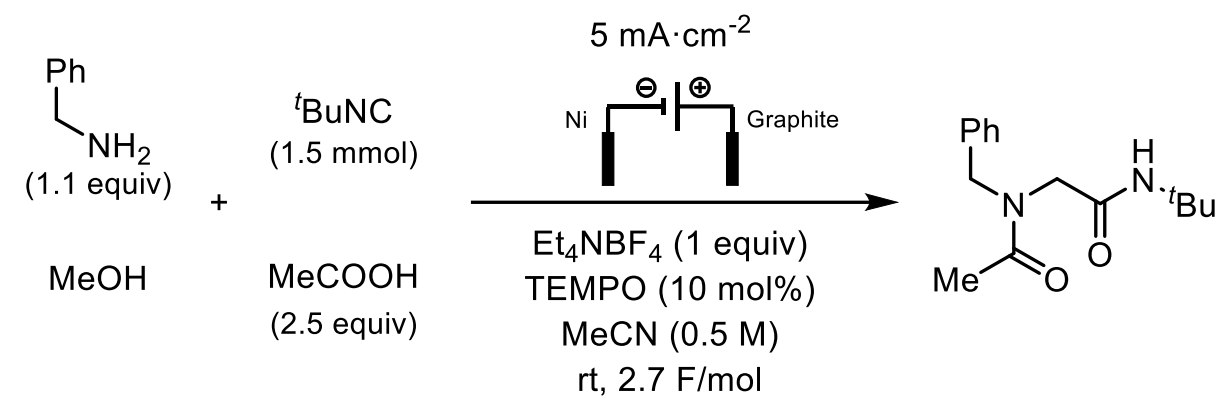

\begin{tabular}{|l|l|l|}
\hline Entry & Alcohol & NMR yield (\%) \\
\hline 1 & 3.0 equiv & 68 \\
\hline 11 & 1.5 equiv & 59 \\
\hline 12 & 6.0 equiv & 74 \\
\hline
\end{tabular}

g) Catalyst

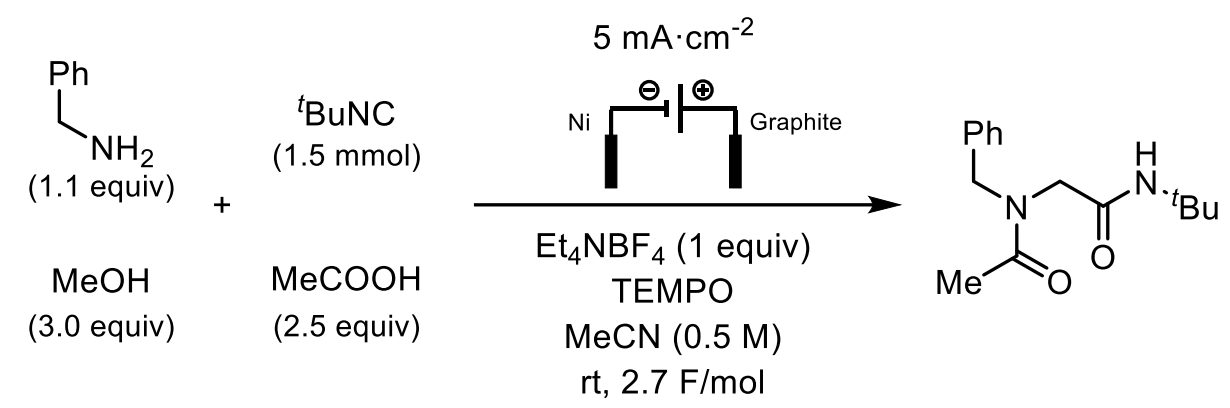

\begin{tabular}{|l|l|l|}
\hline Entry & TEMPO & NMR yield (\%) \\
\hline 1 & $10 \mathrm{~mol} \%$ & $68(70)^{*}$ \\
\hline 13 & $0 \mathrm{~mol} \%$ & 40 \\
\hline 14 & $5 \mathrm{~mol} \%$ & $70(72)^{*}$ \\
\hline 15 & $20 \mathrm{~mol} \%$ & 67 \\
\hline
\end{tabular}

*Isolated yield 
h) Electrolyte

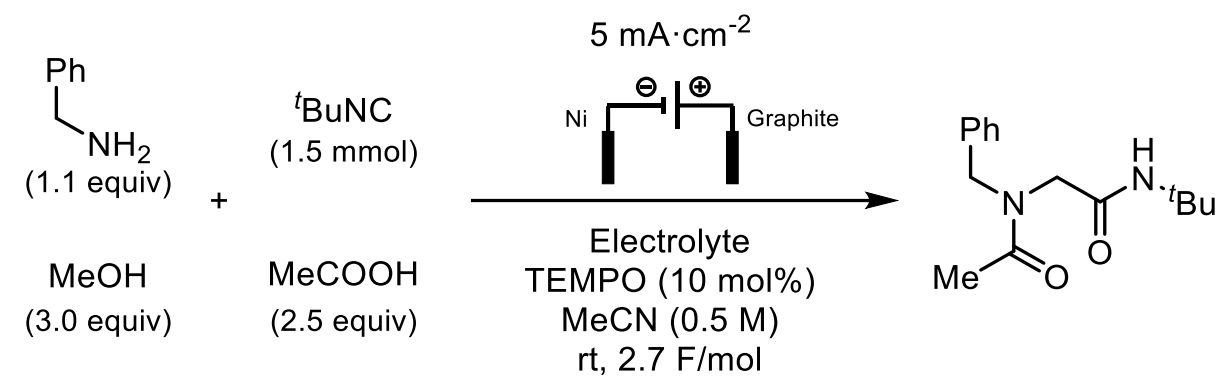

\begin{tabular}{|l|l|l|}
\hline Entry & Electrolyte & NMR yield (\%) \\
\hline 1 & Et$_{4} \mathrm{NBF}_{4}(1 \mathrm{eq})$ & $68(70)^{*}$ \\
\hline 16 & Et$_{4} \mathrm{NOTs}_{(1}$ eq) & 54 \\
\hline 17 & Et$_{4} \mathrm{NPF}_{6}(1 \mathrm{eq})$ & 61 \\
\hline 18 & $/$ & $78(74)^{*}$ \\
\hline
\end{tabular}

*Isolated yield

i) Current density

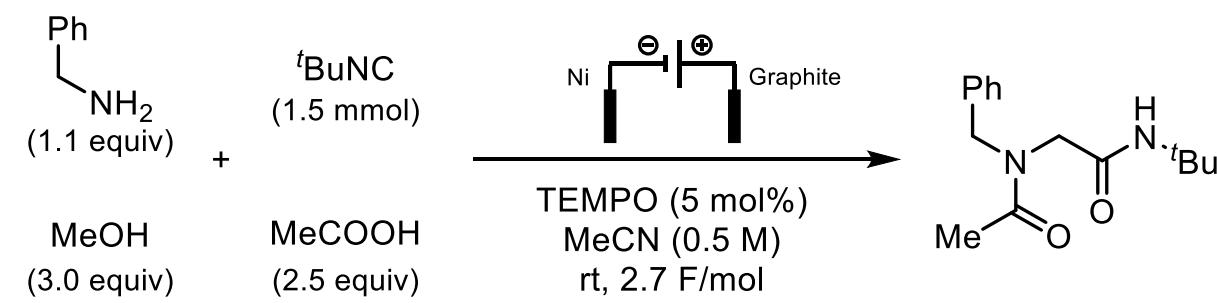

\begin{tabular}{|l|l|l|}
\hline Entry & $\begin{array}{l}\text { Current density }\left(\mathrm{mA} \cdot \mathrm{cm}^{-}\right. \\
2 \mathrm{f}\end{array}$ & NMR yield (\%) \\
\hline 19 & 5 & $76(74)^{*}$ \\
\hline 20 & 7.5 & 74 \\
\hline 21 & $/$ & 0 \\
\hline
\end{tabular}

*Isolated yield

Considering all the data for condition screening, the optimal condition is shown as below. 


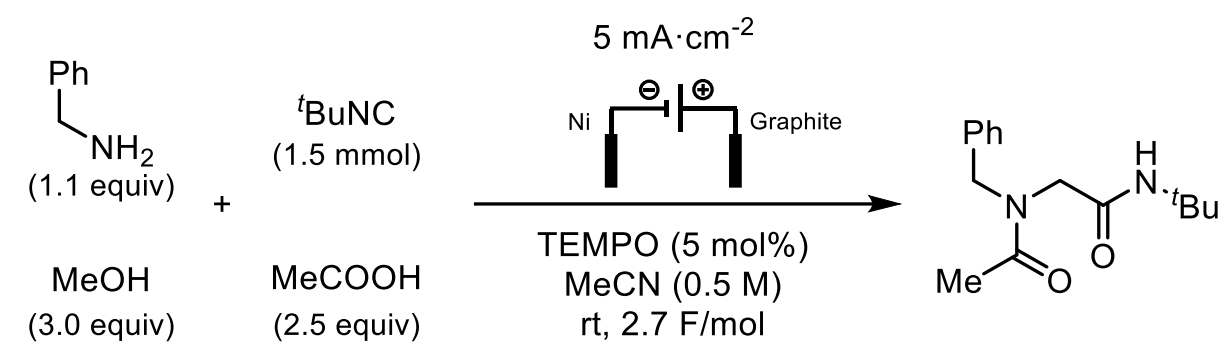

\section{General procedure for electrosynthesis}

TEMPO (12 mg, $0.075 \mathrm{mmol}, 0.05$ equiv), alcohol ( $4.50 \mathrm{mmol}, 3$ equiv), amine (1.65 mmol, 1.1 equiv), carboxylic acid ( $3.75 \mathrm{mmol}, 2.5$ equiv) and isocyanide (1.5 mmol) were dissolved in $3 \mathrm{~mL}$ of anhydrous $\mathrm{CH}_{3} \mathrm{CN}$ in a 5 $\mathrm{mL}$ cell. The cell was then equipped with a graphite anode and a nickel cathode, and electrolysis was performed at a constant current of $14.5 \mathrm{~mA}$ $\left(\sim 5 \mathrm{~mA} / \mathrm{cm}^{2}\right)$ for 7.5 hours $(2.7 \mathrm{~F} / \mathrm{mol})$. After the reaction time indicated, the reaction mixture was transferred into a round bottom flask and concentrated in vacuo. The crude mixture was purified by flash column chromatography to give the desire product. In some cases, the product exists in the form of several rotamers, and changing the deuterated solvent induces a variation in relative proportions.

\section{Characterization of all multicomponent adducts}<smiles>CC(=O)N(CC(=O)NC(C)(C)C)Cc1ccccc1</smiles>

Chemical Formula: $\mathrm{C}_{15} \mathrm{H}_{22} \mathrm{~N}_{2} \mathrm{O}_{2}$

Molecular Weight: 262.35 
Starting from methanol (182 $\mu \mathrm{L}, 4.50 \mathrm{mmol}, 3$ equiv), benzylamine $(180 \mu \mathrm{L}$, 1.65 mmol, 1.1 equiv), acetic acid (215 $\mu \mathrm{L}, 3.75 \mathrm{mmol}, 2.5$ equiv) and tertbutyl isocyanide ( $254 \mu \mathrm{L}, 1.55 \mathrm{mmol})$, and following the general procedure, product 5 (290 mg, 74\% yield) was obtained as a white solid after purification by flash column chromatography. Given the ${ }^{1} \mathrm{H}$ NMR timescale, two rotamers (1.7:1 ratio in $\left.\mathrm{CDCl}_{3}\right)$ were observed. Major rotamer: ${ }^{\mathbf{1}} \mathbf{H}$ NMR (300 MHz, $\mathrm{CDCl}_{3}$ ): $\delta=7.43-7.27(\mathrm{~m}, 4 \mathrm{H}), 7.22-7.15(\mathrm{~m}, 1 \mathrm{H}), 6.11$ (br s, $1 \mathrm{H}$ ), $4.66(\mathrm{~s}, 2 \mathrm{H}), 3.87(\mathrm{~s}, 2 \mathrm{H}), 2.21(\mathrm{~s}, 3 \mathrm{H}), 1.31(\mathrm{~s}, 9 \mathrm{H}) \mathrm{ppm} .{ }^{13} \mathbf{C}\left\{{ }^{\mathbf{1}} \mathbf{H}\right\}$ NMR (75 $\left.\mathrm{MHz}, \mathrm{CDCl}_{3}\right): \delta=172.2,168.4,136.0,129.2,128.0,126.8,53.6,51.5,51.3$, 28.8, 21.6 ppm. HRMS (EI+): calcd for $\mathrm{C}_{15} \mathrm{H}_{22} \mathrm{~N}_{2} \mathrm{O}_{2}$ [M]+: 262.1676, found: 262.1677. Characteristic peaks for the minor rotamer: ${ }^{\mathbf{1}} \mathbf{H}$ NMR $(300 \mathrm{MHz}$, $\left.\mathrm{CDCl}_{3}\right): \delta=5.28($ br s, $1 \mathrm{H}), 4.61(\mathrm{~s}, 2 \mathrm{H}), 3.84(\mathrm{~s}, 2 \mathrm{H}), 2.12(\mathrm{~s}, 3 \mathrm{H}), 1.15(\mathrm{~s}, 9 \mathrm{H})$ ppm. ${ }^{13} \mathbf{C}\left\{{ }^{1} \mathbf{H}\right\}$ NMR $\left(75 \mathrm{MHz}, \mathrm{CDCl}_{3}\right): \delta=171.8,167.2,137.2,129.3,129.0$, $128.3,53.6,51.6,51.2,28.5,21.6 \mathrm{ppm}$.

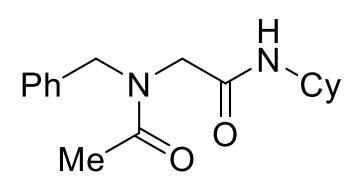

Chemical Formula: $\mathrm{C}_{17} \mathrm{H}_{24} \mathrm{~N}_{2} \mathrm{O}_{2}$

Molecular Weight: 288.39

\section{$N$-benzyl- $N$-(2-(cyclohexylamino)-2-oxoethyl)acetamide ${ }^{1}(6)$}

Starting from methanol (182 $\mu \mathrm{L}, 4.50 \mathrm{mmol}, 3$ equiv), benzylamine $(180 \mu \mathrm{L}$, $1.65 \mathrm{mmol}, 1.1$ equiv), acetic acid (215 $\mu \mathrm{L}, 3.75 \mathrm{mmol}, 2.5$ equiv) and cyclohexyl isocyanide $(280 \mu \mathrm{L}, 1.5 \mathrm{mmol})$, and following the general procedure, product 6 (356 mg, 82\% yield) was obtained as a white solid after purification by flash column chromatography. Given the ${ }^{1} \mathrm{H}$ NMR timescale, two rotamers $\left(2: 1\right.$ ratio in $\left.\mathrm{CDCl}_{3}\right)$ were observed. Major rotamer:

\footnotetext{
${ }^{1}$ Mangunuru, H. P. R.; Yang, H.; Wang, G. Chem. Commun. 2013, 49, 4489-4491.
} 
1H NMR (300 MHz, $\left.\mathrm{CDCl}_{3}\right): \delta=7.42-7.26(\mathrm{~m}, 4 \mathrm{H}), 7.21-7.14(\mathrm{~m}, 1 \mathrm{H}), 6.20$ (br d, $J=6.8 \mathrm{~Hz}, 1 \mathrm{H}), 4.65(\mathrm{~s}, 2 \mathrm{H}), 3.93(\mathrm{~s}, 2 \mathrm{H}), 3.78-3.57(\mathrm{~m}, 1 \mathrm{H}), 2.20(\mathrm{~s}$, $3 \mathrm{H}), 1.90-1.54(\mathrm{~m}, 6 \mathrm{H}), 1.42-1.05(\mathrm{~m}, 4 \mathrm{H})$ ppm. ${ }^{13} \mathbf{C}\left\{{ }^{1} \mathbf{H}\right\}$ NMR $(75 \mathrm{MHz}$, $\left.\mathrm{CDCl}_{3}\right): \delta=172.3,168.2,135.8,129.2,128.1,126.8,53.6,50.7,48.4,32.9$, 25.6, 24.8, 21.6 ppm. HRMS (EI+): calcd for $\mathrm{C}_{17} \mathrm{H}_{24} \mathrm{~N}_{2} \mathrm{O}_{2}[\mathrm{M}]^{+\bullet}:$ 288.1832, found: 288.1836. Characteristic peaks for the minor rotamer: ${ }^{\mathbf{1}} \mathbf{H}$ NMR $(300$ $\mathrm{MHz} \mathrm{CDCl}_{3}$ ): $\delta=5.44(\mathrm{br} \mathrm{d}, J=7.4 \mathrm{~Hz}, 1 \mathrm{H}), 4.61(\mathrm{~s}, 2 \mathrm{H}), 3.90(\mathrm{~s}, 2 \mathrm{H}), 2.12$ (s, 3H), 0.96-0.80 (m, 2H) ppm. ${ }^{13} \mathbf{C}\left\{{ }^{1} \mathbf{H}\right\}$ NMR (75 MHz, $\left.\mathrm{CDCl}_{3}\right): \delta=171.8,167.0$, $137.1,129.2,128.8,128.3,52.8,51.0,25.5,24.9,21.8$ ppm.

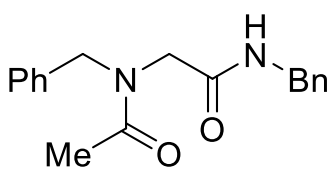

Chemical Formula: $\mathrm{C}_{18} \mathrm{H}_{20} \mathrm{~N}_{2} \mathrm{O}_{2}$

Molecular Weight: 296.37

\section{$N$-benzyl-2-( $N$-benzylacetamido)acetamide (7)}

Starting from methanol (182 $\mu \mathrm{L}, 4.50 \mathrm{mmol}, 3$ equiv), benzylamine (180 $\mu \mathrm{L}$, $1.65 \mathrm{mmol}, 1.1$ equiv), acetic acid ( $215 \mu \mathrm{L}, 3.75 \mathrm{mmol}, 2.5$ equiv) and benzyl isocyanide (183 $\mu \mathrm{L}, 1.5 \mathrm{mmol}$ ), and following the general procedure, product 7 (300 mg, 68\% yield) was obtained as a white solid after purification by flash column chromatography. Given the ${ }^{1} \mathrm{H}$ NMR timescale, two rotamers (3:1 ratio in $\mathrm{CDCl}_{3}$ ) were observed. Major rotamer: ${ }^{\mathbf{1}} \mathbf{H}$ NMR (300 MHz, $\left.\mathrm{CDCl}_{3}\right): \delta=7.41-7.10(\mathrm{~m}, 10 \mathrm{H}), 6.66(\mathrm{br} \mathrm{s}, 1 \mathrm{H}), 4.66(\mathrm{~s}, 2 \mathrm{H}), 4.41$ (d, $J=5.8 \mathrm{~Hz}, 2 \mathrm{H}), 4.00(\mathrm{~s}, 2 \mathrm{H}), 2.20$ (s, 3H) ppm. ${ }^{13} \mathbf{C}\left\{{ }^{1} \mathrm{H}\right\} \mathbf{N M R}(75 \mathrm{MHz}$, $\left.\mathrm{CDCl}_{3}\right): \delta=172.2,169.0,138.1,135.8,129.2,128.8,128.1,127.8,127.6$, 126.7, 53.5, 50.4, 43.5, 21.5 ppm. HRMS (ESI+): calcd for $\mathrm{C}_{18} \mathrm{H}_{21} \mathrm{~N}_{2} \mathrm{O}_{2}$ $[\mathrm{M}+\mathrm{H}]^{+}:$297.1598, found: 297.1602. Characteristic peaks for the minor rotamer: ${ }^{1} \mathbf{H}$ NMR $\left(300 \mathrm{MHz}, \mathrm{CDCl}_{3}\right): \delta=6.03(\mathrm{br} \mathrm{s}, 1 \mathrm{H}), 4.61(\mathrm{~s}, 2 \mathrm{H}), 4.32(\mathrm{~d}$, $J=5.8 \mathrm{~Hz}, 2 \mathrm{H}), 3.94(\mathrm{~s}, 2 \mathrm{H}), 2.11(\mathrm{~s}, 3 \mathrm{H}) \mathrm{ppm} .{ }^{13} \mathbf{C}\left\{{ }^{1} \mathbf{H}\right\} \mathbf{N M R}\left(75 \mathrm{MHz}, \mathrm{CDCl}_{3}\right)$ : 
$\delta=171.6,167.9,137.5,136.8,129.1,129.0,128.6,128.2,127.9,52.2,50.6$, 43.8, $21.9 \mathrm{ppm}$.<smiles>CCCCCCCNC(=O)CN(Cc1ccccc1)C(C)=O</smiles>

Chemical Formula: $\mathrm{C}_{19} \mathrm{H}_{30} \mathrm{~N}_{2} \mathrm{O}_{2}$ Molecular Weight: 318.46

\section{$N$-benzyl- $N$-(2-oxo-2-c(2,4,4-trimethylpentan-2-}

\section{yl)amino)ethyl)acetamide (8)}

Starting from methanol (182 $\mu \mathrm{L}, 4.50 \mathrm{mmol}, 3$ equiv), benzylamine $(180 \mu \mathrm{L}$, $1.65 \mathrm{mmol}, 1.1$ equiv), acetic acid (215 $\mu \mathrm{L}, 3.75 \mathrm{mmol}, 2.5$ equiv) and 1,1,3,3-tetramethylbutyl isocyanide (263 $\mu \mathrm{L}, 1.5 \mathrm{mmol})$, and following the general procedure, product 8 (356 mg, 83\% yield) was obtained as a white solid after purification by flash column chromatography. Given the ${ }^{1} \mathrm{H}$ NMR timescale, two rotamers (2.3:1 ratio in $\left.\mathrm{CDCl}_{3}\right)$ were observed. Major rotamer: ${ }^{\mathbf{1}} \mathbf{H}$ NMR $\left(300 \mathrm{MHz}, \mathrm{CDCl}_{3}\right): \delta=7.41-7.27(\mathrm{~m}, 4 \mathrm{H}), 7.22-7.15(\mathrm{~m}$, 1H), 6.18 (br s, 1H), 4.63 (s, 2H), 3.86 (s, 2H), 2.19 (s, 3H), 1.70 (s, 2H), 1.37 (s, 6H), 0.99 (s, 9H) ppm. ${ }^{13} \mathbf{C}\left\{{ }^{1} \mathbf{H}\right\}$ NMR $\left(75 \mathrm{MHz}, \mathrm{CDCl}_{3}\right): \delta=172.1,167.9$, 135.9, 129.2, 128.0, 126.8, 55.4, 53.4, 51.7, 51.5, 31.7, 31.5, 29.2, 21.5 ppm. HRMS (ESI+): calcd for $\mathrm{C}_{19} \mathrm{H}_{31} \mathrm{~N}_{2} \mathrm{O}_{2}[\mathrm{M}+\mathrm{H}]^{+}$: 319.2380, found: 319.2384. Characteristic peaks for the minor rotamer: ${ }^{\mathbf{1}} \mathbf{H}$ NMR $\left(300 \mathrm{MHz}, \mathrm{CDCl}_{3}\right): \delta=$ 5.41 (br s, 1H), 4.60 (s, 2H), 3.81 (s, 2H), 2.13 (s, 3H), 1.54 (s, 2H), 1.28 (s, 6H), 0.93 (s, 9H) ppm. ${ }^{13} \mathbf{C}\left\{{ }^{1} \mathbf{H}\right\} \mathbf{N M R}\left(75 \mathrm{MHz}, \mathrm{CDCl}_{3}\right): \delta=171.7,166.9$, $137.1,129.2,128.9,128.2,55.7,53.5,52.1,51.0,31.7,28.7,21.9$ ppm.

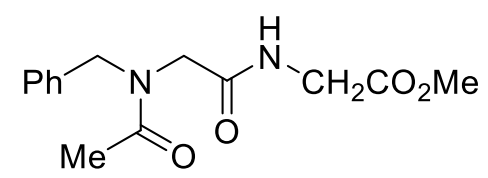

Chemical Formula: $\mathrm{C}_{14} \mathrm{H}_{18} \mathrm{~N}_{2} \mathrm{O}_{4}$ Molecular Weight: 278.31 


\section{Methyl $N$-acetyl- $N$-benzylglycylglycinate (9)}

Starting from methanol $(182 \mu \mathrm{L}, 4.50 \mathrm{mmol}, 3$ equiv), benzylamine $(180 \mu \mathrm{L}$, $1.65 \mathrm{mmol}, 1.1$ equiv), acetic acid (215 $\mu \mathrm{L}, 3.75 \mathrm{mmol}, 2.5$ equiv) and methyl isocyanoacetate $(137 \mu \mathrm{L}, 1.5 \mathrm{mmol})$, and following the general procedure, product 9 (230 mg, 55\% yield) was obtained as a white solid after purification by flash column chromatography. Given the ${ }^{1} \mathrm{H}$ NMR timescale, two rotamers $\left(3: 1\right.$ ratio in $\left.\mathrm{CDCl}_{3}\right)$ were observed. Major rotamer: 1H NMR (300 MHz, $\left.\mathrm{CDCl}_{3}\right): \delta=7.41-7.27(\mathrm{~m}, 4 \mathrm{H}), 7.22-7.16(\mathrm{~m}, 1 \mathrm{H}), 6.78$ (br s, 1H), $4.66(\mathrm{~s}, 2 \mathrm{H}), 4.04-3.96(\mathrm{~m}, 4 \mathrm{H}), 3.76(\mathrm{~s}, 3 \mathrm{H}), 2.24(\mathrm{~s}, 3 \mathrm{H}) \mathrm{ppm}$. ${ }^{13} \mathbf{C}\left\{{ }^{1} \mathrm{H}\right\}$ NMR (75 MHz, $\left.\mathrm{CDCl}_{3}\right): \delta=172.3,170.2,169.3,135.7,129.2,128.1$, 126.8, 53.3, 52.5, 49.9, 41.2, 21.5 ppm. HRMS (ESI+): calcd for $\mathrm{C}_{14} \mathrm{H}_{18} \mathrm{~N}_{2} \mathrm{O}_{4} \mathrm{Na}$ $[\mathrm{M}+\mathrm{Na}]^{+}:$301.1159, found: 301.1159. Characteristic peaks for the minor rotamer: ${ }^{1}$ H NMR (300 MHz, $\mathrm{CDCl}_{3}$ ): $\delta=6.29$ (br s, 1H), 3.93 (s, 2H), 2.19 (s, 3H) ppm. ${ }^{13} \mathbf{C}\left\{{ }^{1} \mathbf{H}\right\}$ NMR $\left(75 \mathrm{MHz}, \mathrm{CDCl}_{3}\right): \delta=136.7,129.0,128.7,52.6,51.5$, $50.1,41.1,21.8 \mathrm{ppm}$.

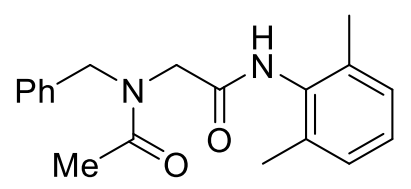

Chemical Formula: $\mathrm{C}_{19} \mathrm{H}_{22} \mathrm{~N}_{2} \mathrm{O}_{2}$

Molecular Weight: 310.40

\section{$N$-benzyl- $N$-(2-((2,6-dimethylphenyl)amino)-2-oxoethyl)acetamide} (10)

Starting from methanol (182 $\mu \mathrm{L}, 4.50 \mathrm{mmol}, 3$ equiv), benzylamine (180 $\mu \mathrm{L}$, $1.65 \mathrm{mmol}, 1.1$ equiv), acetic acid (215 $\mu \mathrm{L}, 3.75 \mathrm{mmol}, 2.5$ equiv) and 2,6dimethylphenyl isocyanoacetate $(197 \mathrm{mg}, 1.5 \mathrm{mmol})$, and following the general procedure, product 10 (240 $\mathrm{mg}, 52 \%$ yield) was obtained as a white solid after purification by flash column chromatography. Given the ${ }^{1} \mathrm{H}$ 
NMR timescale, two rotamers (9:2 ratio in $\mathrm{CDCl}_{3}$ ) were observed. $\underline{\text { Major }}$ rotamer: ${ }^{1} \mathbf{H}$ NMR $\left(300 \mathrm{MHz}, \mathrm{CDCl}_{3}\right): \delta=7.92$ (br s, $\left.1 \mathrm{H}\right), 7.43-7.27(\mathrm{~m}, 4 \mathrm{H})$, 7.25-7.19 (m, 1H), 7.14-7.01 (m, 3H), $4.71(\mathrm{~s}, 2 \mathrm{H}), 4.14(\mathrm{~s}, 2 \mathrm{H}), 2.24(\mathrm{~s}, 3 \mathrm{H})$, $2.18(\mathrm{~s}, 6 \mathrm{H}) \mathrm{ppm} .{ }^{13} \mathrm{C}\left\{{ }^{1} \mathrm{H}\right\}$ NMR $\left(75 \mathrm{MHz}, \mathrm{CDCl}_{3}\right): \delta=172.4,167.7,135.6$, 135.2, 133.7, 129.2, 128.3, 128.2, 127.3, 126.8, 53.5, 50.8, 21.5, 18.5 ppm. HRMS (ESI+): calcd for $\mathrm{C}_{19} \mathrm{H}_{23} \mathrm{~N}_{2} \mathrm{O}_{2}[\mathrm{M}+\mathrm{H}]^{+}:$311.1754, found: 311.1761. Characteristic peaks for the minor rotamer: ${ }^{\mathbf{1}} \mathbf{H}$ NMR $\left(300 \mathrm{MHz}, \mathrm{CDCl}_{3}\right): \delta=$ 7.48 (br s, 1H), 4.72 (s, 2H), 4.03 (s, 2H), 2.25 (s, 3H), 2.13 (s, 6H) ppm. ${ }^{13} \mathbf{C}\left\{{ }^{1} \mathrm{H}\right\}$ NMR $\left(75 \mathrm{MHz}, \mathrm{CDCl}_{3}\right): \delta=171.7,166.6,137.2,136.7,135.3,129.1$, $128.8,128.6,128.5,127.9,51.6,50.4,21.9,18.6 \mathrm{ppm}$.

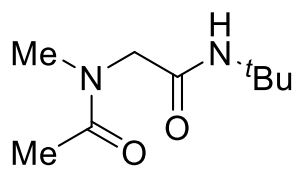

Chemical Formula: $\mathrm{C}_{9} \mathrm{H}_{18} \mathrm{~N}_{2} \mathrm{O}_{2}$

Molecular Weight: 186.26

\section{$N$-(tert-butyl)-2-(N-methylacetamido)acetamide (11)}

Starting from methanol (182 $\mu \mathrm{L}, 4.50 \mathrm{mmol}, 3$ equiv), methylamine ( $40 \%$ wt in $\mathrm{H}_{2} \mathrm{O}$ ) (143 $\mu \mathrm{L}, 1.65 \mathrm{mmol}, 1.1$ equiv), acetic acid (215 $\mu \mathrm{L}, 3.75 \mathrm{mmol}$, 2.5 equiv) and tert-butyl isocyanide (170 $\mu \mathrm{L}, 1.5 \mathrm{mmol})$, and following the general procedure, product 11 (206 $\mathrm{mg}, 74 \%$ yield) was obtained as a white solid after purification by flash column chromatography. Given the ${ }^{1} \mathrm{H}$ NMR timescale, two rotamers (4:1 ratio in $\left.\mathrm{CDCl}_{3}\right)$ were observed. $\underline{\text { Major }}$ rotamer: ${ }^{1} \mathbf{H}$ NMR $\left(300 \mathrm{MHz}, \mathrm{CDCl}_{3}\right): \delta=6.02(\mathrm{br} \mathrm{s}, 1 \mathrm{H}), 3.87(\mathrm{~s}, 2 \mathrm{H}), 3.10$ (s, 3H), $2.14(\mathrm{~m}, 3 \mathrm{H}), 1.32(\mathrm{~s}, 9 \mathrm{H})$ ppm. ${ }^{13} \mathbf{C}\left\{{ }^{\mathbf{1}} \mathbf{H}\right\}$ NMR $\left(75 \mathrm{MHz}, \mathrm{CDCl}_{3}\right): \delta=$ 171.7, 168.3, 53.3, 51.4, 37.7, 28.8, 21.6 ppm. HRMS (ESI+): calcd for $\mathrm{C}_{9} \mathrm{H}_{19} \mathrm{~N}_{2} \mathrm{O}_{2}[\mathrm{M}+\mathrm{H}]^{+}:$187.1441, found: 187.1446. Characteristic peaks for the minor rotamer: ${ }^{1} \mathbf{H}$ NMR $\left(300 \mathrm{MHz}, \mathrm{CDCl}_{3}\right): \delta=5.59(\mathrm{br} \mathrm{s}, 1 \mathrm{H}), 3.84(\mathrm{~s}, 2 \mathrm{H})$, 
$2.97(\mathrm{~s}, 3 \mathrm{H}), 2.08(\mathrm{~m}, 3 \mathrm{H}), 1.36(\mathrm{~s}, 9 \mathrm{H}) \mathrm{ppm} .{ }^{13} \mathbf{C}\left\{{ }^{1} \mathrm{H}\right\} \mathbf{N M R}\left(75 \mathrm{MHz}, \mathrm{CDCl}_{3}\right): \delta$ $=171.6,167.0,55.3,51.9,35.0,28.9,21.5 \mathrm{ppm}$.

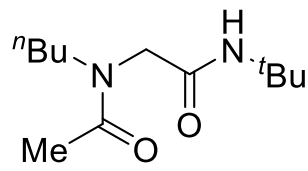

Chemical Formula: $\mathrm{C}_{12} \mathrm{H}_{24} \mathrm{~N}_{2} \mathrm{O}_{2}$

Molecular Weight: 228.34

\section{$N$-(tert-butyl)-2-(N-butylacetamido)acetamide (12)}

Starting from methanol (182 $\mu \mathrm{L}, 4.50 \mathrm{mmol}, 3$ equiv), butylamine (164 $\mu \mathrm{L}$, $1.65 \mathrm{mmol}, 1.1$ equiv), acetic acid (215 $\mu \mathrm{L}, 3.75 \mathrm{mmol}, 2.5$ equiv) and tertbutyl isocyanide (170 $\mu \mathrm{L}, 1.5 \mathrm{mmol}$ ), and following the general procedure, product 12 (264 mg, 77\% yield) was obtained as a white solid after purification by flash column chromatography. Given the ${ }^{1} \mathrm{H}$ NMR timescale, two rotamers (9:2 ratio in $\left.\mathrm{CDCl}_{3}\right)$ were observed. Major rotamer: ${ }^{1} \mathbf{H}$ NMR (300 MHz, $\left.\mathrm{CDCl}_{3}\right): \delta=6.43(\mathrm{br} \mathrm{s}, 1 \mathrm{H}), 3.85(\mathrm{~s}, 2 \mathrm{H}), 3.39-3.31(\mathrm{~m}, 2 \mathrm{H}), 2.14(\mathrm{~s}$, $3 \mathrm{H}), 1.65-1.43(\mathrm{~m}, 2 \mathrm{H}), 1.34-1.25(\mathrm{~m}, 2 \mathrm{H}), 1.31(\mathrm{~s}, 9 \mathrm{H}), 0.94(\mathrm{t}, J=7.3 \mathrm{~Hz}$, 3H) ppm. ${ }^{13} \mathbf{C}\left\{{ }^{1} \mathrm{H}\right\}$ NMR $\left(75 \mathrm{MHz}, \mathrm{CDCl}_{3}\right): \delta=171.6,169.1,52.4,51.2,50.8$, 30.8, 28.7, 21.3, 20.1, 13.9 ppm. HRMS (ESI+): calcd for $\mathrm{C}_{12} \mathrm{H}_{25} \mathrm{~N}_{2} \mathrm{O}_{2}[\mathrm{M}+\mathrm{H}]^{+}$: 229.1911, found: 229.1915. Characteristic peaks for the minor rotamer: ${ }^{\mathbf{1}} \mathbf{H}$ NMR (300 MHz, CDCl $): \delta=5.66$ (br s, 1H), 3.82 (s, 2H), 2.06 (s, 3H), 1.39 (s, 9H) ppm. ${ }^{13} \mathbf{C}\left\{{ }^{1} \mathbf{H}\right\}$ NMR $\left(75 \mathrm{MHz}, \mathrm{CDCl}_{3}\right): \delta=171.4,167.5,53.5,51.9,47.4$, $29.9,28.9,21.9,20.3,13.9 \mathrm{ppm}$.

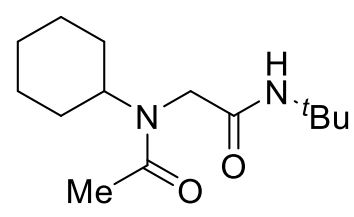

Chemical Formula: $\mathrm{C}_{14} \mathrm{H}_{26} \mathrm{~N}_{2} \mathrm{O}_{2}$

Molecular Weight: 254.37

\section{$N$-(tert-butyl)-2-(N-cyclohexylacetamido)acetamide (13)}


Starting from methanol (182 $\mu \mathrm{L}, 4.50 \mathrm{mmol}, 3$ equiv), cyclohexylamine (189 $\mu \mathrm{L}, 1.65 \mathrm{mmol}, 1.1$ equiv), acetic acid (215 $\mu \mathrm{L}, 3.75 \mathrm{mmol}, 2.5$ equiv) and tert-butyl isocyanide $(170 \mu \mathrm{L}, 1.5 \mathrm{mmol})$, and following the general procedure, product 13 (298 $\mathrm{mg}, 78 \%$ yield) was obtained as a white solid after purification by flash column chromatography. Given the ${ }^{1} \mathrm{H}$ NMR timescale, two rotamers ( $7: 2$ ratio in $\left.\mathrm{CDCl}_{3}\right)$ were observed. Major rotamer: ${ }^{1} \mathbf{H}$ NMR (300 MHz, $\mathrm{CDCl}_{3}$ ): $\delta=6.60$ (br s, $\left.1 \mathrm{H}\right), 3.81(\mathrm{~s}, 2 \mathrm{H}), 3.52(\mathrm{tt}, J=11.7$, $3.8 \mathrm{~Hz}, 1 \mathrm{H}), 2.17(\mathrm{~s}, 3 \mathrm{H}), 1.89-1.37(\mathrm{~m}, 8 \mathrm{H}), 1.29$ (s, 9H), 1.26-1.01 (m, 2H) ppm. ${ }^{13} \mathbf{C}\left\{{ }^{1} \mathrm{H}\right\}$ NMR $\left(75 \mathrm{MHz}, \mathrm{CDCl}_{3}\right): \delta=171.6,170.0,58.9,50.9,47.6,31.2$, 28.7, 25.9, 25.1, 21.8 ppm. HRMS (ESI+): calcd for $\mathrm{C}_{14} \mathrm{H}_{27} \mathrm{~N}_{2} \mathrm{NaO}_{2}[\mathrm{M}+\mathrm{H}]^{+}$: 255.2067, found: 255.2067. Characteristic peaks for the minor rotamer: ${ }^{1} \mathbf{H}$ NMR (300 MHz, $\mathrm{CDCl}_{3}$ ): $\delta=5.76(\mathrm{br} \mathrm{s}, 1 \mathrm{H}), 4.53-4.38(\mathrm{~m}, 1 \mathrm{H}), 3.73(\mathrm{~s}, 2 \mathrm{H})$, $2.04(\mathrm{~s}, 3 \mathrm{H}), 1.34(\mathrm{~s}, 9 \mathrm{H}) \mathrm{ppm} .{ }^{13} \mathbf{C}\left\{{ }^{1} \mathrm{H}\right\}$ NMR $\left(75 \mathrm{MHz}, \mathrm{CDCl}_{3}\right): \delta=171.7$, $168.4,53.2,51.7,48.6,30.5,28.8,25.7,25.4,22.5$ ppm.

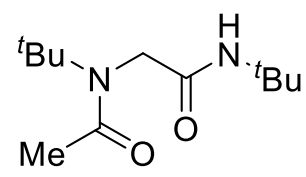

Chemical Formula: $\mathrm{C}_{12} \mathrm{H}_{24} \mathrm{~N}_{2} \mathrm{O}_{2}$

Molecular Weight: 228.34

\section{$N$-(tert-butyl)-2-(N-(tert-butyl)acetamido)acetamide (14)}

Starting from methanol (182 $\mu \mathrm{L}, 4.50 \mathrm{mmol}, 3$ equiv), tert-butylamine (174 $\mu \mathrm{L}, 1.65 \mathrm{mmol}, 1.1$ equiv), acetic acid (215 $\mu \mathrm{L}, 3.75 \mathrm{mmol}, 2.5$ equiv) and tert-butyl isocyanide $(170 \mu \mathrm{L}, 1.5 \mathrm{mmol})$, and following the general procedure, product 14 (301 $\mathrm{mg}$, 88\% yield) was obtained as a white solid after purification by flash column chromatography. ${ }^{1} \mathbf{H}$ NMR $(300 \mathrm{MHz}$, $\mathrm{CDCl}_{3}$ ): $\delta=5.91$ (br s, 1H), $3.84(\mathrm{~s}, 2 \mathrm{H}), 2.07$ (s, 3H), $1.44(\mathrm{~s}, 9 \mathrm{H}), 1.37(\mathrm{~m}$, 9H) ppm. ${ }^{13} \mathbf{C}\left\{{ }^{1} \mathbf{H}\right\}$ NMR $\left(75 \mathrm{MHz}, \mathrm{CDCl}_{3}\right): \delta=172.5,169.0,57.9,51.6,51.0$, 
28.9, 28.8, 25.4 ppm. HRMS (ESI+): calcd for $\mathrm{C}_{12} \mathrm{H}_{24} \mathrm{~N}_{2} \mathrm{NaO}_{2}[\mathrm{M}+\mathrm{Na}]^{+}$: 251.1730, found: 251.1733 .

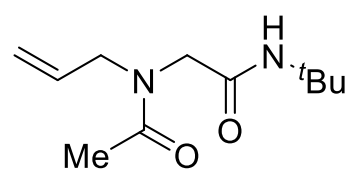

Chemical Formula: $\mathrm{C}_{11} \mathrm{H}_{20} \mathrm{~N}_{2} \mathrm{O}_{2}$

Molecular Weight: 212.29

\section{$N$-allyl- $N$-(2-(tert-butylamino)-2-oxoethyl)acetamide (15)}

Starting from methanol (182 $\mu \mathrm{L}, 4.50 \mathrm{mmol}, 3$ equiv), allylamine $(124 \mu \mathrm{L}$, $1.65 \mathrm{mmol}, 1.1$ equiv), acetic acid (215 $\mu \mathrm{L}, 3.75 \mathrm{mmol}, 2.5$ equiv) and tertbutyl isocyanide (170 $\mu \mathrm{L}, 1.5 \mathrm{mmol}$ ), and following the general procedure, product 15 (270 mg, 85\% yield) was obtained as a colorless oil after purification by flash column chromatography. Given the ${ }^{1} \mathrm{H}$ NMR timescale, two rotamers $\left(7: 2\right.$ ratio in $\left.\mathrm{CDCl}_{3}\right)$ were observed. Major rotamer: ${ }^{1} \mathbf{H}$ NMR (300 MHz, $\mathrm{CDCl}_{3}$ ): $\delta=6.17$ (br s, $1 \mathrm{H}$ ), 5.79 (ddd, $J=15.5,10.0,5.0 \mathrm{~Hz}, 1 \mathrm{H}$ ), $5.23(\mathrm{~d}, J=10.0 \mathrm{~Hz}, 1 \mathrm{H}), 5.17$ (d, $J=15.5 \mathrm{~Hz}, 1 \mathrm{H}), 4.02(\mathrm{~d}, J=5.0 \mathrm{~Hz}, 2 \mathrm{H}$ ), $3.85(\mathrm{~s}, 2 \mathrm{H}), 2.14(\mathrm{~s}, 3 \mathrm{H}), 1.32(\mathrm{~s}, 9 \mathrm{H}) \mathrm{ppm} .{ }^{13} \mathbf{C}\left\{{ }^{1} \mathrm{H}\right\} \mathbf{N M R}\left(75 \mathrm{MHz}, \mathrm{CDCl}_{3}\right): \delta$ $=171.9,168.5,132.0,117.5,52.6,51.6,51.3,28.8,21.3$ ppm. HRMS (ESI+): calcd for $\mathrm{C}_{11} \mathrm{H}_{21} \mathrm{~N}_{2} \mathrm{O}_{2}[\mathrm{M}+\mathrm{H}]^{+}:$213.1598, found: 213.1599. Characteristic peaks for the minor rotamer: ${ }^{1} \mathbf{H}$ NMR $\left(300 \mathrm{MHz}, \mathrm{CDCl}_{3}\right): \delta=5.64(\mathrm{br} \mathrm{s}, 1 \mathrm{H})$, $3.81(\mathrm{~s}, 2 \mathrm{H}), 2.09(\mathrm{~s}, 3 \mathrm{H}), 1.36(\mathrm{~s}, 9 \mathrm{H}) \mathrm{ppm} .{ }^{13} \mathbf{C}\left\{{ }^{1} \mathrm{H}\right\} \mathbf{N M R}\left(75 \mathrm{MHz}, \mathrm{CDCl}_{3}\right): \delta$ $=171.4,167.3,132.6,119.1,52.7,51.9,50.0,28.9,21.8$ ppm.

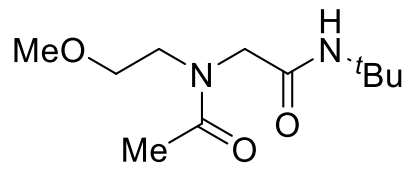

Chemical Formula: $\mathrm{C}_{11} \mathrm{H}_{22} \mathrm{~N}_{2} \mathrm{O}_{3}$ Molecular Weight: 230.31 


\section{$N$-(tert-butyl)-2-(N-(2-methoxyethyl)acetamido)acetamide (16)}

Starting from methanol (182 $\mu \mathrm{L}, 4.50 \mathrm{mmol}, 3$ equiv), 2-methoxyethylamine (144 $\mu \mathrm{L}, 1.65 \mathrm{mmol}, 1.1$ equiv), acetic acid (215 $\mu \mathrm{L}, 3.75 \mathrm{mmol}, 2.5$ equiv) and tert-butyl isocyanide $(170 \mu \mathrm{L}, 1.5 \mathrm{mmol})$, and following the general procedure, product 16 (234 mg, 68\% yield) was obtained as a yellowish oil after purification by flash column chromatography. Given the ${ }^{1} \mathrm{H}$ NMR timescale, two rotamers (11:10 ratio in $\left.\mathrm{CDCl}_{3}\right)$ were observed. Major rotamer: ${ }^{1} \mathbf{H}$ NMR $\left(300 \mathrm{MHz}, \mathrm{CDCl}_{3}\right): \delta=6.39(\mathrm{br} \mathrm{s}, 1 \mathrm{H}), 3.86(\mathrm{~s}, 2 \mathrm{H}), 3.63(\mathrm{~s}$, $3 \mathrm{H}), 3.63-3.49(\mathrm{~m}, 2 \mathrm{H}), 3.34(\mathrm{~s}, 3 \mathrm{H}), 2.18(\mathrm{~s}, 3 \mathrm{H}), 1.32(\mathrm{~s}, 9 \mathrm{H}) \mathrm{ppm} .{ }^{13} \mathbf{C}\left\{{ }^{1} \mathbf{H}\right\}$ NMR (75 MHz, $\left.\mathrm{CDCl}_{3}\right): \delta=172.5,168.9,70.2,59.2,52.6,51.2,50.7,28.8$, 21.7 ppm. HRMS (ESI+): calcd for $\mathrm{C}_{11} \mathrm{H}_{23} \mathrm{~N}_{2} \mathrm{O}_{3}[\mathrm{M}+\mathrm{H}]^{+}:$:231.1703, found: 231.1703. Characteristic peaks for the minor rotamer: ${ }^{\mathbf{1}} \mathbf{H}$ NMR $(300 \mathrm{MHz}$, $\mathrm{CDCl}_{3}$ ): $\delta=6.79$ (br s, $\left.1 \mathrm{H}\right), 3.89(\mathrm{~s}, 2 \mathrm{H}), 3.37(\mathrm{~s}, 3 \mathrm{H}), 2.85(\mathrm{~s}, 3 \mathrm{H}), 1.35(\mathrm{~s}, 9 \mathrm{H})$ ppm. ${ }^{13} \mathbf{C}\left\{{ }^{1} \mathbf{H}\right\}$ NMR $\left(75 \mathrm{MHz}, \mathrm{CDCl}_{3}\right): \delta=172.2,168.3,70.2,58.6,55.9,51.4$, 48.7, 28.8, 22.0.

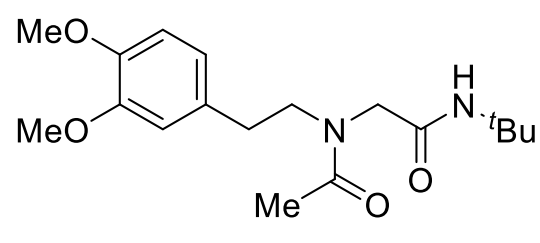

Chemical Formula: $\mathrm{C}_{18} \mathrm{H}_{28} \mathrm{~N}_{2} \mathrm{O}_{4}$

Molecular Weight: 336.43

\section{$N$-(tert-butyl)-2-(N-(3,4-dimethoxyphenethyl)acetamido)acetamide} (17)

Starting from methanol (182 $\mu \mathrm{L}, 4.50 \mathrm{mmol}, 3$ equiv), 3,4dimethoxyphenethylamine (278 $\mu \mathrm{L}, 1.65 \mathrm{mmol}, 1.1$ equiv), acetic acid (215 $\mu \mathrm{L}, 3.75 \mathrm{mmol}, 2.5$ equiv) and tert-butyl isocyanide (170 $\mu \mathrm{L}, 1.5 \mathrm{mmol})$, and following the general procedure, product 17 (440 $\mathrm{mg}, 87 \%$ yield) was obtained as a white solid after purification by flash column chromatography. 
Given the ${ }^{1} \mathrm{H}$ NMR timescale, two rotamers $\left(10: 3\right.$ ratio in $\left.\mathrm{CDCl}_{3}\right)$ were observed. Major rotamer: ${ }^{1} \mathbf{H}$ NMR $\left(300 \mathrm{MHz}, \mathrm{CDCl}_{3}\right): \delta=6.83-6.65(\mathrm{~m}, 3 \mathrm{H})$, 6.38 (br s, 1H), 3.90-3.84 (m, 8H), 3.59 (t, $J=7.3 \mathrm{~Hz}, 2 \mathrm{H}), 2.82(\mathrm{t}, J=7.3 \mathrm{~Hz}$, 2H), 1.97 (s, 3H), 1.32 (s, 9H) ppm. ${ }^{13} \mathbf{C}\left\{{ }^{1} \mathbf{H}\right\}$ NMR (75 MHz, $\left.\mathrm{CDCl}_{3}\right): \delta=171.7$, 168.8, 149.3, 148.1, 130.5, 121.0, 112.1, 111.6, 56.1, 56.0, 52.5, 52.3, 51.3, 34.7, 28.8, 21.1 ppm. HRMS (ESI+): calcd for $\mathrm{C}_{18} \mathrm{H}_{29} \mathrm{~N}_{2} \mathrm{O}_{4}[\mathrm{M}+\mathrm{H}]^{+}: 337.2122$, found: 337.2129. Characteristic peaks for the minor rotamer: ${ }^{1} \mathbf{H}$ NMR (300 $\mathrm{MHz}_{\mathrm{CDCl}}$ ): $\delta=5.55$ (br s, 1H), $3.72(\mathrm{~s}, 2 \mathrm{H}), 2.07$ (s, 3H), 1.32 (s, 9H) ppm. ${ }^{13} \mathbf{C}\left\{{ }^{1} \mathrm{H}\right\}$ NMR $\left(75 \mathrm{MHz}, \mathrm{CDCl}_{3}\right): \delta=171.4,167.3,149.2,147.9,131.1,120.7$, $112.0,111.5,53.9,51.8,49.5,33.5,21.9$ ppm.

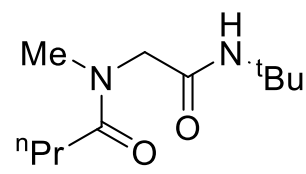

Chemical Formula: $\mathrm{C}_{11} \mathrm{H}_{22} \mathrm{~N}_{2} \mathrm{O}_{2}$

Molecular Weight: 214.31

\section{$N$-(2-(tert-butylamino)-2-oxoethyl)- $N$-methylbutyramide (18)}

Starting from methanol (182 $\mu \mathrm{L}, 4.50 \mathrm{mmol}, 3$ equiv), methylamine ( $40 \%$ wt in $\mathrm{H}_{2} \mathrm{O}$ ) (143 $\mu \mathrm{L}, 1.65 \mathrm{mmol}, 1.1$ equiv), butyric acid (342 $\mu \mathrm{L}, 3.75 \mathrm{mmol}$, 2.5 equiv) and tert-butyl isocyanide (170 $\mu \mathrm{L}, 1.5 \mathrm{mmol})$, and following the general procedure, product 18 (266 $\mathrm{mg}, 83 \%$ yield) was obtained as a yellow oil after purification by flash column chromatography. Given the ${ }^{1} \mathrm{H}$ NMR timescale, two rotamers (4:1 ratio in $\left.\mathrm{CDCl}_{3}\right)$ were observed. $\underline{\text { Major }}$ rotamer: ${ }^{1} \mathrm{H}$ NMR $\left(300 \mathrm{MHz}, \mathrm{CDCl}_{3}\right): \delta=6.10(\mathrm{br} \mathrm{s}, 1 \mathrm{H}), 3.88(\mathrm{~s}, 2 \mathrm{H}), 3.10(\mathrm{~s}$, $3 \mathrm{H}), 2.35(\mathrm{t}, J=7.4 \mathrm{~Hz}, 2 \mathrm{H}), 1.75-1.61(\mathrm{~m}, 2 \mathrm{H}), 1.31(\mathrm{~s}, 9 \mathrm{H}), 0.98(\mathrm{t}, J=7.4$ $\mathrm{Hz}, 3 \mathrm{H}) \mathrm{ppm} .{ }^{13} \mathbf{C}\left\{{ }^{1} \mathrm{H}\right\}$ NMR $\left(75 \mathrm{MHz}, \mathrm{CDCl}_{3}\right): \delta=174.2,168.6,53.7,51.3$, 37.0, 35.2, 28.8, 18.6, 14.0 ppm. HRMS (ESI+): calcd for $\mathrm{C}_{14} \mathrm{H}_{27} \mathrm{~N}_{2} \mathrm{NaO}_{2}$ $[\mathrm{M}+\mathrm{Na}]^{+}:$237.1573, found: 237.1572. Characteristic peaks for the minor 
rotamer: ${ }^{1} \mathrm{H}$ NMR $\left(300 \mathrm{MHz}, \mathrm{CDCl}_{3}\right): \delta=5.58(\mathrm{br} \mathrm{s}, 1 \mathrm{H}), 3.85(\mathrm{~s}, 2 \mathrm{H}), 2.98(\mathrm{~s}$, $3 \mathrm{H}), 2.24(\mathrm{t}, J=7.4 \mathrm{~Hz}, 2 \mathrm{H}), 1.36(\mathrm{~s}, 9 \mathrm{H}) \mathrm{ppm}$.

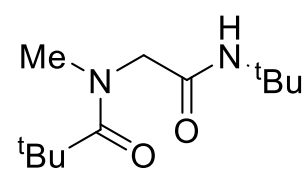

Chemical Formula: $\mathrm{C}_{12} \mathrm{H}_{24} \mathrm{~N}_{2} \mathrm{O}_{2}$

Molecular Weight: 228.34

\section{$N$-(2-(tert-butylamino)-2-oxoethyl)- $N$-methylpivalamide (19)}

Starting from methanol (182 $\mu \mathrm{L}, 4.50 \mathrm{mmol}, 3$ equiv), methylamine ( $40 \%$ wt in $\mathrm{H}_{2} \mathrm{O}$ ) (143 $\mu \mathrm{L}, 1.65 \mathrm{mmol}, 1.1$ equiv), pivalic acid (383 mg, $3.75 \mathrm{mmol}$, 2.5 equiv) and tert-butyl isocyanide (170 $\mu \mathrm{L}, 1.5 \mathrm{mmol})$, and following the general procedure, product 19 (242 $\mathrm{mg}, 71 \%$ yield) was obtained as a white solid after purification by flash column chromatography. ${ }^{1} \mathbf{H}$ NMR (300 MHz, $\mathrm{CDCl}_{3}$ ): $\delta=6.06$ (br s, $\left.1 \mathrm{H}\right), 3.87(\mathrm{~s}, 2 \mathrm{H}), 3.16(\mathrm{~s}, 3 \mathrm{H}), 1.31(\mathrm{~s}, 9 \mathrm{H})$, 1.29 (s, 9H) ppm. ${ }^{13} \mathbf{C}\left\{{ }^{1} \mathbf{H}\right\}$ NMR $\left(75 \mathrm{MHz}, \mathrm{CDCl}_{3}\right): \delta=178.6,168.6,55.7,51.2$, 38.9, 38.0, 28.8, 28.1 ppm. HRMS (ESI+): calcd for $\mathrm{C}_{12} \mathrm{H}_{25} \mathrm{~N}_{2} \mathrm{O}_{2}[\mathrm{M}+\mathrm{H}]^{+}$: 229.1911, found: 229.1912 .

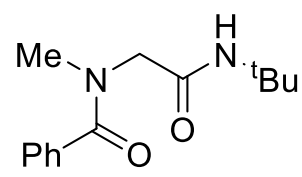

Chemical Formula: $\mathrm{C}_{14} \mathrm{H}_{20} \mathrm{~N}_{2} \mathrm{O}_{2}$

Molecular Weight: 248.3260

\section{$N$-(2-(tert-butylamino)-2-oxoethyl)- $N$-methylbenzamide (20)}

Starting from methanol (182 $\mu \mathrm{L}, 4.50 \mathrm{mmol}, 3$ equiv), methylamine ( $40 \%$ wt in $\mathrm{H}_{2} \mathrm{O}$ ) (143 $\mu \mathrm{L}, 1.65 \mathrm{mmol}, 1.1$ equiv), benzoic acid (458 mg, $3.75 \mathrm{mmol}$, 2.5 equiv) and tert-butyl isocyanide (170 $\mu \mathrm{L}, 1.5 \mathrm{mmol})$, and following the general procedure, product 20 (300 $\mathrm{mg}, 81 \%$ yield) was obtained as a white solid after purification by flash column chromatography. ${ }^{1} \mathbf{H}$ NMR 
(300 MHz, $\left.\mathrm{CDCl}_{3}\right): \delta=7.53-7.32(\mathrm{~m}, 5 \mathrm{H}), 6.30(\mathrm{br} \mathrm{s}, 1 \mathrm{H}), 4.04(\mathrm{~s}, 2 \mathrm{H}), 3.08$ $(\mathrm{s}, 3 \mathrm{H}), 1.36(\mathrm{~s}, 9 \mathrm{H}) \mathrm{ppm} .{ }^{13} \mathbf{C}\left\{{ }^{1} \mathrm{H}\right\} \mathbf{N M R}\left(75 \mathrm{MHz}, \mathrm{CDCl}_{3}\right): \delta=\mathrm{s}$ 172.4, 168.1, 135.3, 130.3, 128.7, 127.3, 53.5, 51.4, 39.0, 28.9 ppm. HRMS (ESI+): calcd for $\mathrm{C}_{14} \mathrm{H}_{21} \mathrm{~N}_{2} \mathrm{O}_{2}[\mathrm{M}+\mathrm{H}]^{+}:$249.1598, found: 249.1596 .

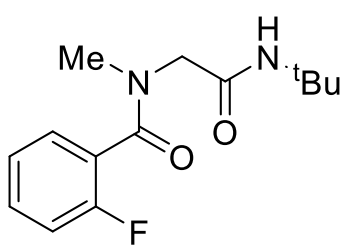

Chemical Formula: $\mathrm{C}_{14} \mathrm{H}_{19} \mathrm{FN}_{2} \mathrm{O}_{2}$

Molecular Weight: 266.32

\section{$N$-(2-(tert-butylamino)-2-oxoethyl)-2-fluoro- $N$-methylbenzamide (21)}

Starting from methanol (182 $\mu \mathrm{L}, 4.50 \mathrm{mmol}, 3$ equiv), methylamine ( $40 \%$ wt in $\mathrm{H}_{2} \mathrm{O}$ ) (143 $\mu \mathrm{L}, 1.65 \mathrm{mmol}, 1.1$ equiv), 2-fluorobenzoic acid (525 mg, $3.75 \mathrm{mmol}, 2.5$ equiv) and tert-butyl isocyanide (170 $\mu \mathrm{L}, 1.5 \mathrm{mmol})$, and following the general procedure, product 21 (316 $\mathrm{mg}, 79 \%$ yield) was obtained as a yellowish liquid after purification by flash column chromatography. Given the ${ }^{1} \mathrm{H}$ NMR timescale, two rotamers $(4: 1$ ratio in $\left.\mathrm{CDCl}_{3}\right)$ were observed. Major rotamer: ${ }^{1} \mathrm{H}$ NMR $\left(300 \mathrm{MHz}, \mathrm{CDCl}_{3}\right): \delta=7.49$ 7.37 (m, 2H), 7.27-7.20 (m, 1H), 7.17-7.08 (m, 1H), 6.17 (br s, 1H), 4.11 (s, 2H), $3.00(\mathrm{~d}, J=1.4 \mathrm{~Hz}, 3 \mathrm{H}), 1.37(\mathrm{~s}, 9 \mathrm{H})$ ppm. ${ }^{13} \mathbf{C}\left\{{ }^{1} \mathbf{H}\right\} \mathbf{N M R}(75 \mathrm{MHz}$, $\left.\mathrm{CDCl}_{3}\right): \delta=167.5,167.4,158.4\left(J_{C-F}=246.7 \mathrm{~Hz}\right), 131.9\left(U_{C-F}=8.2 \mathrm{~Hz}\right), 129.0$ $\left.U_{C-F}=3.6 \mathrm{~Hz}\right), 125.1\left(U_{C-F}=3.4 \mathrm{~Hz}\right), 123.8\left(U_{C-F}=17.2 \mathrm{~Hz}\right), 115.9 U_{C-F}=21.5$ $\mathrm{Hz}), 52.9,51.5,37.7,28.8$ ppm. ${ }^{19} \mathbf{F}\left\{{ }^{1} \mathbf{H}\right\}$ NMR $\left(282 \mathrm{MHz}, \mathrm{CDCl}_{3}\right): \delta=-116.1$ ppm. HRMS (ESI+): calcd for $\mathrm{C}_{14} \mathrm{H}_{20} \mathrm{FN}_{2} \mathrm{O}_{2}[\mathrm{M}+\mathrm{H}]^{+}:$267.1503, found: 267.1506. Characteristic peaks for the minor rotamer: ${ }^{1} \mathbf{H}$ NMR $(300 \mathrm{MHz}$, $\left.\mathrm{CDCl}_{3}\right): \delta=5.62(\mathrm{br} \mathrm{s}, 1 \mathrm{H}), 3.75(\mathrm{~s}, 2 \mathrm{H}), 3.15(\mathrm{~s}, 3 \mathrm{H}), 1.34(\mathrm{~s}, 9 \mathrm{H}) \mathrm{ppm}$. ${ }^{13} \mathbf{C}\left\{{ }^{1} \mathbf{H}\right\}$ NMR (75 MHz, $\left.\mathrm{CDCl}_{3}\right): \delta=55.7,51.8,34.3$ ppm. ${ }^{19} \mathbf{F}\left\{{ }^{\mathbf{1}} \mathbf{H}\right\}$ NMR $(282$ $\left.\mathrm{MHz}, \mathrm{CDCl}_{3}\right): \delta=-114.8 \mathrm{ppm}$. 


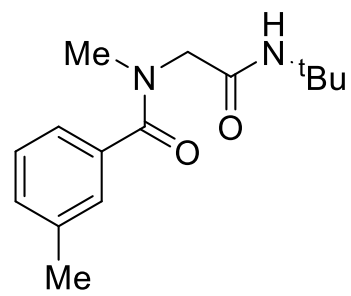

Chemical Formula: $\mathrm{C}_{15} \mathrm{H}_{22} \mathrm{~N}_{2} \mathrm{O}_{2}$

Molecular Weight: 262.3530

\section{$N$-(2-(tert-butylamino)-2-oxoethyl)- $N, 3$-dimethylbenzamide (22)}

Starting from methanol (182 $\mu \mathrm{L}, 4.50 \mathrm{mmol}, 3$ equiv), methylamine ( $40 \%$ wt in $\mathrm{H}_{2} \mathrm{O}$ ) (143 $\mu \mathrm{L}, 1.65 \mathrm{mmol}, 1.1$ equiv), $m$-toluic acid (510 mg, 3.75 mmol, 2.5 equiv) and tert-butyl isocyanide (170 $\mu \mathrm{L}, 1.5 \mathrm{mmol})$, and following the general procedure, product 22 (296 $\mathrm{mg}, 75 \%$ yield) was obtained as a colorless oil after purification by flash column chromatography. Given the ${ }^{1} \mathrm{H}$ NMR timescale, two rotamers $3: 1$ ratio in $\left.\mathrm{CDCl}_{3}\right)$ were observed. Major rotamer: ${ }^{1} \mathrm{H}$ NMR $\left(300 \mathrm{MHz}, \mathrm{CDCl}_{3}\right): \delta=7.35$ 7.16 (m, 4H), 6.34 (br s, 1H), 4.04 (s, 2H), 3.08 (s, 3H), 2.38 (s, 3H), 1.37 (s, 9H) ppm. ${ }^{13} \mathbf{C}\left\{{ }^{1} \mathbf{H}\right\}$ NMR $\left(75 \mathrm{MHz}, \mathrm{CDCl}_{3}\right): \delta=172.6,168.2,138.6,135.3$, 131.0, 128.5, 127.8, 124.2, 53.5, 51.4, 39.0, 28.9, 21.5 ppm. HRMS (ESI+): calcd for $\mathrm{C}_{15} \mathrm{H}_{23} \mathrm{~N}_{2} \mathrm{O}_{2}[\mathrm{M}+\mathrm{H}]^{+}:$263.1754, found: 263.1754. Characteristic peaks for the minor rotamer: ${ }^{1} \mathbf{H}$ NMR $\left(300 \mathrm{MHz}, \mathrm{CDCl}_{3}\right): \delta=5.52(\mathrm{br} \mathrm{s}, 1 \mathrm{H})$, $3.81(\mathrm{~s}, 2 \mathrm{H}) \mathrm{ppm}$.

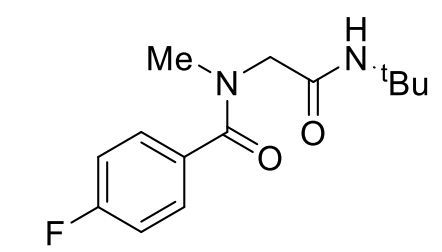

Chemical Formula: $\mathrm{C}_{14} \mathrm{H}_{19} \mathrm{FN}_{2} \mathrm{O}_{2}$ Molecular Weight: 266.32 
Starting from methanol (182 $\mu \mathrm{L}, 4.50 \mathrm{mmol}, 3$ equiv), methylamine ( $40 \%$ wt in $\mathrm{H}_{2} \mathrm{O}$ ) (143 $\mu \mathrm{L}, 1.65 \mathrm{mmol}, 1.1$ equiv), 4-fluorobenzoic acid (525 mg, $3.75 \mathrm{mmol}, 2.5$ equiv) and tert-butyl isocyanide (170 $\mu \mathrm{L}, 1.5 \mathrm{mmol})$, and following the general procedure, product 23 (284 $\mathrm{mg}, 71 \%$ yield) was obtained as a white solid after purification by flash column chromatography. 1H NMR (300 MHz, $\mathrm{CDCl}_{3}$ ): $\delta=7.46(\mathrm{dd}, J=7.6,5.8 \mathrm{~Hz}, 2 \mathrm{H}), 7.09(\mathrm{t}, J=8.1$ $\mathrm{Hz}, 2 \mathrm{H}$ ), 6.22 (br s, 1H), 4.01 (s, 2H), 3.08 (s, 3H), 1.35 (s, 9H) ppm. ${ }^{13} \mathbf{C}\left\{{ }^{1} \mathrm{H}\right\}$ NMR (75 MHz, $\left.\mathrm{CDCl}_{3}\right): \delta=171.5,167.9,163.8\left(\mathrm{JCF}_{C-F}=250.5 \mathrm{~Hz}\right), 131.4,129.7$ $\left(U_{C-F}=5.6 \mathrm{~Hz}\right), 115.7\left(J_{C-F}=21.8 \mathrm{~Hz}\right), 53.4,51.5,39.1,28.9$ ppm. ${ }^{19} \mathbf{F}\left\{{ }^{1} \mathbf{H}\right\} \mathbf{~ N M R}$ (282 MHz, $\mathrm{CDCl}_{3}$ ): $\delta=-109.5 \mathrm{ppm}$. HRMS (ESI+): calcd for $\mathrm{C}_{14} \mathrm{H}_{20} \mathrm{FN}_{2} \mathrm{O}_{2}$ $[\mathrm{M}+\mathrm{H}]^{+}:$267.1503, found: 267.1505. Characteristic peaks for the minor rotamer: ${ }^{19} \mathbf{F}\left\{{ }^{1} \mathbf{H}\right\}$ NMR $\left(282 \mathrm{MHz}, \mathrm{CDCl}_{3}\right): \delta=-109.7 \mathrm{ppm}$.

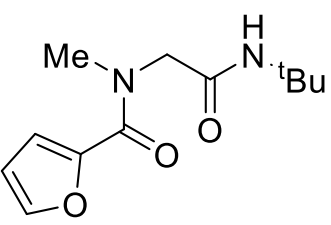

Chemical Formula: $\mathrm{C}_{12} \mathrm{H}_{18} \mathrm{~N}_{2} \mathrm{O}_{3}$

Molecular Weight: 238.29

\section{$N$-(2-(tert-butylamino)-2-oxoethyl)- $N$-methylfuran-2-carboxamide (24)}

Starting from methanol (182 $\mu \mathrm{L}, 4.50 \mathrm{mmol}, 3$ equiv), methylamine (40\% wt in $\mathrm{H}_{2} \mathrm{O}$ ) (143 $\mu \mathrm{L}, 1.65 \mathrm{mmol}, 1.1$ equiv), 2-furoic acid (420 mg, $3.75 \mathrm{mmol}$, 2.5 equiv) and tert-butyl isocyanide (170 $\mu \mathrm{L}, 1.5 \mathrm{mmol})$, and following the general procedure, product 24 (241 $\mathrm{mg}$, 68\% yield) was obtained as a white solid after purification by flash column chromatography. ${ }^{\mathbf{1}} \mathbf{H}$ NMR (300 MHz, $\left.\mathrm{CDCl}_{3}\right): \delta=7.59-7.44(\mathrm{~m}, 1 \mathrm{H}), 7.09$ (d, $\left.J=3.5 \mathrm{~Hz}, 1 \mathrm{H}\right)$, 6.56-6.45 (m, 1H), 6.21 (br s, 1H), 4.04 (s, 2H), 3.32 (br s, 3H), 1.34 (s, 9H) ppm. ${ }^{13} \mathbf{C}\left\{{ }^{1} \mathrm{H}\right\}$ NMR $\left(75 \mathrm{MHz}, \mathrm{CDCl}_{3}\right): \delta=168.0,160.9,147.4,144.6,117.5,111.7$, 54.9, 51.5, 37.7, 28.8 ppm. HRMS (ESI+): calcd for $\mathrm{C}_{12} \mathrm{H}_{19} \mathrm{~N}_{2} \mathrm{O}_{3}[\mathrm{M}+\mathrm{H}]^{+}$: 227.1754, found: 227.1754 . 


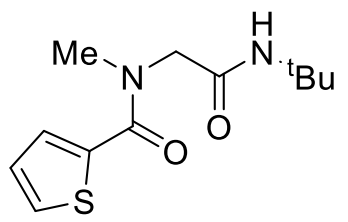

Chemical Formula: $\mathrm{C}_{12} \mathrm{H}_{18} \mathrm{~N}_{2} \mathrm{O}_{2} \mathrm{~S}$

Molecular Weight: 254.35

\section{$N$-(2-(tert-butylamino)-2-oxoethyl)- $N$-methylthiophene-2- carboxamide (25)}

Starting from methanol (182 $\mu \mathrm{L}, 4.50 \mathrm{mmol}, 3$ equiv), methylamine ( $40 \%$ wt in $\mathrm{H}_{2} \mathrm{O}$ ) (143 $\mu \mathrm{L}, 1.65 \mathrm{mmol}, 1.1$ equiv), 2-thiophenecarboxylic acid (480 $\mathrm{mg}, 3.75 \mathrm{mmol}, 2.5$ equiv) and tert-butyl isocyanide (170 $\mu \mathrm{L}, 1.5 \mathrm{mmol})$, and following the general procedure, product 25 (267 $\mathrm{mg}, 70 \%$ yield) was obtained as a white solid after purification by flash column chromatography. ${ }^{1} \mathbf{H}$ NMR (300 MHz, $\left.\mathrm{CDCl}_{3}\right): \delta=7.49(\mathrm{~d}, J=4.9 \mathrm{~Hz}, 1 \mathrm{H}), 7.41(\mathrm{~d}, J=3.4 \mathrm{~Hz}$, 1H), 7.06 (dd, J = 4.9, $3.9 \mathrm{~Hz}, 1 \mathrm{H}), 6.16(\mathrm{br} \mathrm{s}, 1 \mathrm{H}), 4.04(\mathrm{~s}, 2 \mathrm{H}), 3.28(\mathrm{~s}, 3 \mathrm{H})$, $1.35(\mathrm{~s}, 9 \mathrm{H}) \mathrm{ppm} .{ }^{13} \mathbf{C}\left\{{ }^{1} \mathrm{H}\right\}$ NMR $\left(75 \mathrm{MHz}, \mathrm{CDCl}_{3}\right): \delta=167.8,165.2,137.0$, 130.1 (2C), 127.2, 55.1, 51.6, 38.1, 28.8 ppm. HRMS (ESI+): calcd for $\mathrm{C}_{12} \mathrm{H}_{19} \mathrm{~N}_{2} \mathrm{O}_{2} \mathrm{~S}[\mathrm{M}+\mathrm{H}]^{+}:$255.1162, found: 255.1162 .

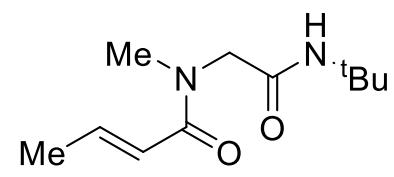

Chemical Formula: $\mathrm{C}_{11} \mathrm{H}_{20} \mathrm{~N}_{2} \mathrm{O}_{2}$

Molecular Weight: 212.29

\section{(E)- $N$-(2-(tert-butylamino)-2-oxoethyl)- $N$-methylbut-2-enamide (26)}

Starting from methanol (182 $\mu \mathrm{L}, 4.50 \mathrm{mmol}, 3$ equiv), methylamine ( $40 \%$ wt in $\mathrm{H}_{2} \mathrm{O}$ ) (143 $\mu \mathrm{L}, 1.65 \mathrm{mmol}, 1.1$ equiv), crotonic acid (323 mg, 3.75 mmol, 2.5 equiv) and tert-butyl isocyanide (170 $\mu \mathrm{L}, 1.5 \mathrm{mmol})$, and following the general procedure, product 26 (243 $\mathrm{mg}, 76 \%$ yield) was obtained as a white solid after purification by flash column chromatography. 
Given the ${ }^{1} \mathrm{H}$ NMR timescale, two rotamers (3:1 ratio in $\mathrm{CDCl}_{3}$ ) were observed. Major rotamer: ${ }^{1} \mathbf{H}$ NMR $\left(300 \mathrm{MHz}, \mathrm{CDCl}_{3}\right): \delta=6.94(\mathrm{dq}, J=13.8$, $6.9 \mathrm{~Hz}, 1 \mathrm{H}), 6.35-6.00(\mathrm{~m}, 2 \mathrm{H}), 3.91(\mathrm{~s}, 2 \mathrm{H}), 3.13(\mathrm{~s}, 3 \mathrm{H}), 1.94-1.83(\mathrm{~m}, 3 \mathrm{H})$, $1.30(\mathrm{~s}, 9 \mathrm{H}) \mathrm{ppm} .{ }^{13} \mathbf{C}\left\{{ }^{1} \mathbf{H}\right\}$ NMR $\left(75 \mathrm{MHz}, \mathrm{CDCl}_{3}\right): \delta=168.5,167.5,143.2$, 121.1, 53.9, 51.2, 36.8, 28.8, 18.4 ppm. HRMS (EI+): calcd for $\mathrm{C}_{11} \mathrm{H}_{21} \mathrm{~N}_{2} \mathrm{O}_{2}$ $[\mathrm{M}+\mathrm{H}]^{+}:$213.1598, found: 213.1597. Characteristic peaks for the minor rotamer: ${ }^{1} \mathbf{H}$ NMR $\left(300 \mathrm{MHz}, \mathrm{CDCl}_{3}\right): \delta=5.67$ (br s, $\left.1 \mathrm{H}\right), 3.01$ (s, 3H) ppm. ${ }^{13} \mathbf{C}\left\{{ }^{1} \mathrm{H}\right\}$ NMR (75 MHz, $\left.\mathrm{CDCl}_{3}\right): \delta=167.7,143.7,120.8,54.6,51.8,35.4$ ppm.

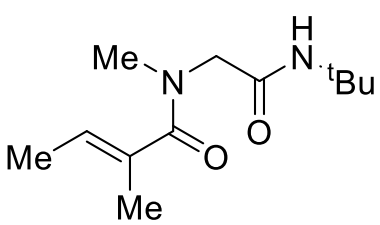

Chemical Formula: $\mathrm{C}_{12} \mathrm{H}_{22} \mathrm{~N}_{2} \mathrm{O}_{2}$

Molecular Weight: 226.32

\section{(E)-N-(2-(tert-butylamino)-2-oxoethyl)- $N, 2$-dimethylbut-2-enamide} (27)

Starting from methanol (182 $\mu \mathrm{L}, 4.50 \mathrm{mmol}, 3$ equiv), methylamine (40\% wt in $\mathrm{H}_{2} \mathrm{O}$ ) (143 $\mu \mathrm{L}, 1.65 \mathrm{mmol}, 1.1$ equiv), tiglic acid (375 mg, $3.75 \mathrm{mmol}$, 2.5 equiv) and tert-butyl isocyanide $(170 \mu \mathrm{L}, 1.5 \mathrm{mmol})$, and following the general procedure, product 27 (236 $\mathrm{mg}, 70 \%$ yield) was obtained as a colorless oil after purification by flash column chromatography. ${ }^{\mathbf{1}} \mathbf{H}$ NMR $\left(300 \mathrm{MHz}, \mathrm{CDCl}_{3}\right): \delta=6.18(\mathrm{br} \mathrm{s}, 1 \mathrm{H}), 5.70(\mathrm{qd}, J=6.8,1.5 \mathrm{~Hz}, 1 \mathrm{H}), 3.90(\mathrm{~s}$, $2 \mathrm{H}), 3.05(\mathrm{~s}, 3 \mathrm{H}), 1.87-1.81(\mathrm{~m}, 3 \mathrm{H}), 1.72(\mathrm{dd}, J=6.8,1.1 \mathrm{~Hz}, 3 \mathrm{H}), 1.34(\mathrm{~s}$, 9H) ppm. ${ }^{13} \mathbf{C}\left\{{ }^{1} \mathrm{H}\right\}$ NMR $\left(75 \mathrm{MHz}, \mathrm{CDCl}_{3}\right): \delta=173.7,167.1,130.7,126.2,52.6$, 50.4, 37.1, 27.9, 13.0, 12.4 ppm. HRMS (EI+): calcd for $\mathrm{C}_{12} \mathrm{H}_{23} \mathrm{~N}_{2} \mathrm{O}_{2}[\mathrm{M}+\mathrm{H}]^{+}$: 327.1754, found: 227.1754 . 


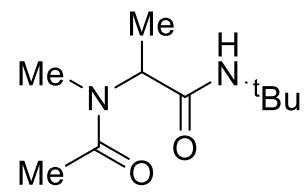

Chemical Formula: $\mathrm{C}_{10} \mathrm{H}_{20} \mathrm{~N}_{2} \mathrm{O}_{2}$

Molecular Weight: 200.28

\section{$N$-(tert-butyl)-2-( $N$-methylacetamido)propanamide (28)}

Starting from ethanol ( $262 \mu \mathrm{L}, 4.50 \mathrm{mmol}, 3$ equiv), methylamine ( $40 \% \mathrm{wt}$ in $\mathrm{H}_{2} \mathrm{O}$ ) (143 $\mu \mathrm{L}, 1.65 \mathrm{mmol}, 1.1$ equiv), acetic acid (225 mg, $3.75 \mathrm{mmol}, 2.5$ equiv) and tert-butyl isocyanide $(170 \mu \mathrm{L}, 1.5 \mathrm{mmol})$, and following the general procedure, product 28 (202 $\mathrm{mg}, 67 \%$ yield) was obtained as a white solid after purification by flash column chromatography. Given the ${ }^{1} \mathrm{H}$ NMR timescale, two rotamers (8:1 ratio in $\mathrm{CDCl}_{3}$ ) were observed. Major rotamer: ${ }^{1} \mathbf{H}$ NMR $\left(300 \mathrm{MHz}, \mathrm{CDCl}_{3}\right): \delta=5.99(\mathrm{br} \mathrm{s}, 1 \mathrm{H}), 5.06(\mathrm{q}, J=7.1 \mathrm{~Hz}$, 1H), $2.90(\mathrm{~s}, 3 \mathrm{H}), 2.12(\mathrm{~s}, 3 \mathrm{H}), 1.30(\mathrm{~s}, 9 \mathrm{H}), 1.26(\mathrm{~d}, J=7.1 \mathrm{~Hz}, 3 \mathrm{H}) \mathrm{ppm}$. ${ }^{13} \mathbf{C}\left\{{ }^{1} \mathrm{H}\right\}$ NMR $\left(75 \mathrm{MHz}, \mathrm{CDCl}_{3}\right): \delta=171.6,170.4,52.1,51.2,31.1,28.8,22.2$, 13.3 ppm. HRMS (ESI+): calcd for $\mathrm{C}_{10} \mathrm{H}_{20} \mathrm{~N}_{2} \mathrm{O}_{2} \mathrm{Na}$ [M+Na] $]^{+}: 223.1417$, found: 223.1414. Characteristic peaks for the minor rotamer: ${ }^{1} \mathbf{H}$ NMR $(300 \mathrm{MHz}$, $\left.\mathrm{CDCl}_{3}\right): \delta=2.80(\mathrm{~s}, 3 \mathrm{H}), 2.15(\mathrm{~s}, 3 \mathrm{H}), 1.34(\mathrm{~s}, 9 \mathrm{H}) \mathrm{ppm} .{ }^{13} \mathbf{C}\left\{{ }^{\mathbf{1}} \mathbf{H}\right\} \mathbf{N M R}(75$ $\mathrm{MHz}, \mathrm{CDCl}_{3}$ ): $\delta=58.2,28.7 \mathrm{ppm}$.

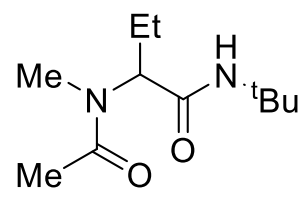

Chemical Formula: $\mathrm{C}_{11} \mathrm{H}_{22} \mathrm{~N}_{2} \mathrm{O}_{2}$

Molecular Weight: 214.31

\section{$N$-(tert-butyl)-2-( $N$-methylacetamido)butanamide (29)}

Starting from 1-propanol (336 $\mu \mathrm{L}, 4.50 \mathrm{mmol}, 3$ equiv), methylamine ( $40 \%$ wt in $\mathrm{H}_{2} \mathrm{O}$ ) (143 $\mu \mathrm{L}, 1.65 \mathrm{mmol}, 1.1$ equiv), acetic acid (225 mg, $3.75 \mathrm{mmol}$, 2.5 equiv) and tert-butyl isocyanide (170 $\mu \mathrm{L}, 1.5 \mathrm{mmol})$, and following the 
general procedure, product 29 (170 $\mathrm{mg}, 53 \%$ yield) was obtained as a yellow oil after purification by flash column chromatography. Given the ${ }^{1} \mathrm{H}$ NMR timescale, two rotamers (9:1 ratio in $\mathrm{CDCl}_{3}$ ) were observed. $\underline{\text { Major }}$ rotamer: ${ }^{1} \mathbf{H}$ NMR $\left(300 \mathrm{MHz}, \mathrm{CDCl}_{3}\right): \delta=5.96(\mathrm{br} \mathrm{s}, 1 \mathrm{H}), 4.79(\mathrm{t}, J=7.8 \mathrm{~Hz}$, 1H), 2.91 (s, 3H), 2.13 (s, 3H), 1.98-1.81 (m, 1H), 1.66-1.54 (m, 1H), 1.30 (s, 9H), $0.85(\mathrm{t}, J=7.4 \mathrm{~Hz}, 3 \mathrm{H}) \mathrm{ppm} .{ }^{13} \mathbf{C}\left\{{ }^{1} \mathbf{H}\right\} \mathbf{N M R}\left(75 \mathrm{MHz}, \mathrm{CDCl}_{3}\right): \delta=172.0$, 169.9, 58.2, 51.2, 31.2, 28.8, 22.2, 20.8, 10.6 ppm. HRMS (ESI+): calcd for $\mathrm{C}_{11} \mathrm{H}_{22} \mathrm{~N}_{2} \mathrm{O}_{2} \mathrm{Na}[\mathrm{M}+\mathrm{Na}]^{+}:$237.1573, found: 237.1572. Characteristic peaks for the minor rotamer: ${ }^{1} \mathrm{H}$ NMR (300 MHz, $\mathrm{CDCl}_{3}$ ): $\delta=5.57$ (br s, 1H), 2.79 (s, $3 \mathrm{H}), 2.15(\mathrm{~s}, 3 \mathrm{H}), 1.34(\mathrm{~s}, 9 \mathrm{H}) \mathrm{ppm}$.

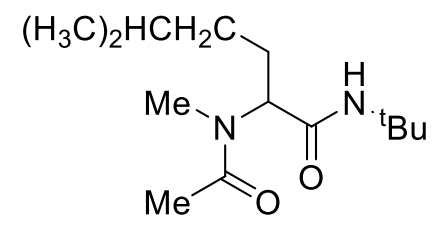

Chemical Formula: $\mathrm{C}_{14} \mathrm{H}_{28} \mathrm{~N}_{2} \mathrm{O}_{2}$

Molecular Weight: 256.39

\section{$N$-(tert-butyl)-5-methyl-2-( $N$-methylacetamido)hexanamide (30)}

Starting from 4-methyl-1-pentanol (560 $\mu \mathrm{L}, 4.50 \mathrm{mmol}, 3$ equiv), methylamine (40\% wt in $\mathrm{H}_{2} \mathrm{O}$ ) (143 $\mu \mathrm{L}, 1.65 \mathrm{mmol}, 1.1$ equiv), acetic acid (225 mg, $3.75 \mathrm{mmol}, 2.5$ equiv) and tert-butyl isocyanide (170 $\mu \mathrm{L}, 1.5$ mmol), and following the general procedure, product 30 (236 $\mathrm{mg}, 61 \%$ yield) was obtained as a yellow oil after purification by flash column chromatography. Given the ${ }^{1} \mathrm{H}$ NMR timescale, two rotamers $10: 1$ ratio in $\mathrm{CDCl}_{3}$ ) were observed. Major rotamer: ${ }^{1} \mathbf{H}$ NMR $\left(300 \mathrm{MHz}, \mathrm{CDCl}_{3}\right): \delta=5.94$ (br s, 1H), 4.84 (dd, $J=8.6,6.9 \mathrm{~Hz}, 1 \mathrm{H}), 2.91$ (s, 3H), 2.13 (s, 3H), 1.92-1.76 (m, 1H), 1.65-1.49 (m, 2H), 1.30 (s, 9H), 1.21-0.94 (m, 2H), 0.88 (d, $J=6.6$ $\mathrm{Hz}, 1 \mathrm{H}) .{ }^{13} \mathbf{C}\left\{{ }^{1} \mathbf{H}\right\}$ NMR $\left(75 \mathrm{MHz}, \mathrm{CDCl}_{3}\right): \delta=172.0,170.1,56.9,51.2,35.1$, 31.2, 28.9, 28.1, 25.3, 22.8, 22.5, 22.3 ppm. HRMS (ESI+): calcd for $\mathrm{C}_{14} \mathrm{H}_{29} \mathrm{~N}_{2} \mathrm{O}_{2}[\mathrm{M}+\mathrm{H}]^{+}:$257.2224, found: 257.2222. Characteristic peaks for the 
minor rotamer: ${ }^{1} \mathbf{H}$ NMR $\left(300 \mathrm{MHz}, \mathrm{CDCl}_{3}\right): \delta=5.57(\mathrm{br} \mathrm{s}, 1 \mathrm{H}), 2.79(\mathrm{~s}, 3 \mathrm{H})$, $1.34(\mathrm{~s}, 9 \mathrm{H}) \mathrm{ppm}$.

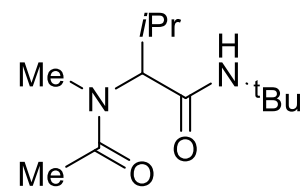

Chemical Formula: $\mathrm{C}_{12} \mathrm{H}_{24} \mathrm{~N}_{2} \mathrm{O}_{2}$

Molecular Weight: 228.34

\section{$N$-(tert-butyl)-3-methyl-2-( $N$-methylacetamido)butanamide (31)}

Starting from isobutanol ( $416 \mu \mathrm{L}, 4.50 \mathrm{mmol}, 3$ equiv), methylamine ( $40 \%$ wt in $\mathrm{H}_{2} \mathrm{O}$ ) (143 $\mu \mathrm{L}, 1.65 \mathrm{mmol}, 1.1$ equiv), acetic acid (225 mg, $3.75 \mathrm{mmol}$, 2.5 equiv) and tert-butyl isocyanide (170 $\mu \mathrm{L}, 1.5 \mathrm{mmol})$, and following the general procedure, product 31 (237 $\mathrm{mg}$, 69\% yield) was obtained as a white solid after purification by flash column chromatography. ${ }^{\mathbf{1}} \mathbf{H}$ NMR (300 MHz, $\left.\mathrm{CDCl}_{3}\right): \delta=5.89($ br s, $1 \mathrm{H}), 4.39(\mathrm{~d}, J=11.2 \mathrm{~Hz}, 1 \mathrm{H}), 2.97(\mathrm{~s}, 3 \mathrm{H})$, 2.31-2.18 (m, 1H), $2.12(\mathrm{~s}, 3 \mathrm{H}), 1.31(\mathrm{~s}, 9 \mathrm{H}), 0.95(\mathrm{~d}, J=6.5 \mathrm{~Hz}, 3 \mathrm{H}), 0.81(\mathrm{~d}, J$ $=6.7 \mathrm{~Hz}, 3 \mathrm{H})$ ppm. ${ }^{13} \mathbf{C}\left\{{ }^{1} \mathrm{H}\right\}$ NMR $\left(75 \mathrm{MHz}, \mathrm{CDCl}_{3}\right): \delta=172.1,169.5,63.3$, 51.3, 31.5, 28.8, 26.0, 22.2, 19.7, 18.7 ppm. HRMS (ESI+): calcd for $\mathrm{C}_{12} \mathrm{H}_{25} \mathrm{~N}_{2} \mathrm{O}_{2}[\mathrm{M}+\mathrm{H}]^{+}:$229.1911, found: 229.1910.

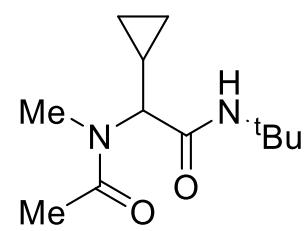

Chemical Formula: $\mathrm{C}_{12} \mathrm{H}_{22} \mathrm{~N}_{2} \mathrm{O}_{2}$

Molecular Weight: 226.32

\section{$N$-(tert-butyl)-2-cyclopropyl-2-( $N$-methylacetamido)acetamide (32)}

Starting from cyclopropanemethanol (364 $\mu \mathrm{L}, 4.50 \mathrm{mmol}, 3$ equiv), methylamine ( $40 \%$ wt in $\mathrm{H}_{2} \mathrm{O}$ ) (143 $\mu \mathrm{L}, 1.65$ mmol, 1.1 equiv), acetic acid (225 mg, $3.75 \mathrm{mmol}, 2.5$ equiv) and tert-butyl isocyanide (170 $\mu \mathrm{L}, 1.5$ $\mathrm{mmol}$ ), and following the general procedure, product 32 (198 mg, 58\% 
yield) was obtained as a white solid after purification by flash column chromatography. ${ }^{1} \mathbf{H}$ NMR (300 MHz, $\mathrm{CDCl}_{3}$ ): $\delta=6.02$ (br s, $1 \mathrm{H}$ ), 4.03 (d, $J=$ $10.1 \mathrm{~Hz}, 1 \mathrm{H}), 3.06$ (s, 3H), 2.12 (s, 3H), 1.38-1.34 (m, 1H), $1.31(\mathrm{~s}, 9 \mathrm{H}), 0.82-$ $0.69(\mathrm{~m}, 1 \mathrm{H}), 0.61-0.48(\mathrm{~m}, 1 \mathrm{H}), 0.41(\mathrm{td}, J=9.6,4.6 \mathrm{~Hz}, 1 \mathrm{H}), 0.23(\mathrm{dt}, J=9.6$, $5.2 \mathrm{~Hz}, 1 \mathrm{H}) \mathrm{ppm} .{ }^{13} \mathbf{C}\left\{{ }^{1} \mathrm{H}\right\} \mathbf{N M R}\left(75 \mathrm{MHz}, \mathrm{CDCl}_{3}\right): \delta=171.6,169.6,62.7,51.3$, 32.4, 28.9, 22.0, 9.9, 6.1, 2.8 ppm. HRMS (ESI+): calcd for $\mathrm{C}_{12} \mathrm{H}_{23} \mathrm{~N}_{2} \mathrm{O}_{2}$ $[\mathrm{M}+\mathrm{H}]^{+}:$227.1754, found: 227.1753.

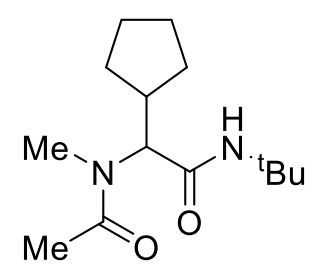

Chemical Formula: $\mathrm{C}_{14} \mathrm{H}_{26} \mathrm{~N}_{2} \mathrm{O}_{2}$

Molecular Weight: 254.37

\section{$N$-(tert-butyl)-2-cyclopentyl-2-( $N$-methylacetamido)acetamide (33)}

Starting from cyclopentanemethanol (487 $\mu \mathrm{L}, 4.50 \mathrm{mmol}, 3$ equiv), methylamine ( $40 \%$ wt in $\mathrm{H}_{2} \mathrm{O}$ ) (143 $\mu \mathrm{L}, 1.65 \mathrm{mmol}, 1.1$ equiv), acetic acid (225 mg, $3.75 \mathrm{mmol}, 2.5$ equiv) and tert-butyl isocyanide (170 $\mu \mathrm{L}, 1.5$ $\mathrm{mmol}$ ), and following the general procedure, product $33(222 \mathrm{mg}, 58 \%$ yield) was obtained as a white solid after purification by flash column chromatography. Given the ${ }^{1} \mathrm{H}$ NMR timescale, two rotamers $30: 1$ ratio in $\mathrm{CDCl}_{3}$ ) were observed. Major rotamer: ${ }^{1} \mathbf{H}$ NMR (300 MHz, $\mathrm{CDCl}_{3}$ ): $\delta=5.94$ (br s, 1H), 4.50 (d, J = 11.4 Hz, 1H), 2.96 (s, 3H), 2.59-2.41 (m, 1H), 2.11 (s, $3 \mathrm{H}), 1.92-1.76(\mathrm{~m}, 1 \mathrm{H}), 1.68-1.48(\mathrm{~m}, 5 \mathrm{H}), 1.29(\mathrm{~s}, 9 \mathrm{H}), 1.22-1.04(\mathrm{~m}, 2 \mathrm{H})$ ppm. ${ }^{13} \mathbf{C}\left\{{ }^{1} \mathrm{H}\right\}$ NMR $\left(75 \mathrm{MHz}, \mathrm{CDCl}_{3}\right): \delta=171.9,169.9,61.8,51.3,37.3,31.7$, 30.4, 29.0, 28.9, 25.8, 25.5, 22.2 ppm. HRMS (ESI+): calcd for $\mathrm{C}_{14} \mathrm{H}_{27} \mathrm{~N}_{2} \mathrm{O}_{2}$ $[\mathrm{M}+\mathrm{H}]^{+}:$255.2067, found: 255.2075. Characteristic peaks for the minor rotamer: ${ }^{1} \mathbf{H}$ NMR $\left(300 \mathrm{MHz}, \mathrm{CDCl}_{3}\right): \delta=2.86(\mathrm{~s}, 3 \mathrm{H}), 2.16(\mathrm{~s}, 3 \mathrm{H}), 1.33(\mathrm{~s}, 9 \mathrm{H})$ ppm. 


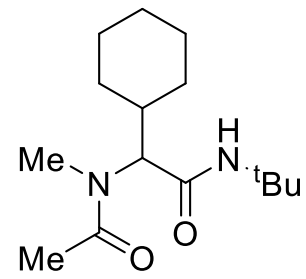

Chemical Formula: $\mathrm{C}_{15} \mathrm{H}_{28} \mathrm{~N}_{2} \mathrm{O}_{2}$

Molecular Weight: 268.40

\section{$N$-(tert-butyl)-2-cyclohexyl-2-( $N$-methylacetamido)acetamide (34)}

Starting from cyclohexanemethanol (553 $\mu \mathrm{L}, 4.50 \mathrm{mmol}, 3$ equiv), methylamine ( $40 \%$ wt in $\mathrm{H}_{2} \mathrm{O}$ ) (143 $\mu \mathrm{L}, 1.65 \mathrm{mmol}, 1.1$ equiv), acetic acid (225 mg, $3.75 \mathrm{mmol}, 2.5$ equiv) and tert-butyl isocyanide $(170 \mu \mathrm{L}, 1.5$ mmol), and following the general procedure, product 34 (258 $\mathrm{mg}, 64 \%$ yield) was obtained as a white solid after purification by flash column chromatography. Given the ${ }^{1} \mathrm{H}$ NMR timescale, two rotamers $(13: 1$ ratio in $\mathrm{CDCl}_{3}$ ) were observed. Major rotamer: ${ }^{\mathbf{1}} \mathbf{H}$ NMR $\left(300 \mathrm{MHz}, \mathrm{CDCl}_{3}\right): \delta=5.87$ (br s, 1H), 4.50 (d, J = $11.1 \mathrm{~Hz}, 1 \mathrm{H}), 2.98$ (s, 3H), 2.13 (s, 3H), 2.04-1.89 (m, 1H), 1.83-1.64 (m, 4H), 1.55-1.45 (m, 1H), $1.36(\mathrm{~s}, 9 \mathrm{H}), 1.36-1.17(\mathrm{~m}, 3 \mathrm{H})$, 1.01-0.82 (m, 2H) ppm. ${ }^{13} \mathbf{C}\left\{{ }^{1} \mathrm{H}\right\}$ NMR $\left(75 \mathrm{MHz}, \mathrm{CDCl}_{3}\right): \delta=172.0,169.3$, 61.9, 51.4, 35.1, 31.6, 30.1, 29.0, 28.8, 26.5, 25.8, 22.2 ppm. HRMS (ESI+): calcd for $\mathrm{C}_{15} \mathrm{H}_{29} \mathrm{~N}_{2} \mathrm{O}_{2}[\mathrm{M}+\mathrm{H}]^{+}:$269.2242, found: 269.2242. Characteristic peaks for the minor rotamer: ${ }^{\mathbf{1}} \mathbf{H}$ NMR $\left(300 \mathrm{MHz}, \mathrm{CDCl}_{3}\right): \delta=2.91(\mathrm{~s}, 3 \mathrm{H})$, $2.17(\mathrm{~s}, 3 \mathrm{H}) \mathrm{ppm} .{ }^{13} \mathbf{C}\left\{{ }^{1} \mathbf{H}\right\}$ NMR $\left(75 \mathrm{MHz}, \mathrm{CDCl}_{3}\right): \delta=28.7 \mathrm{ppm}$.

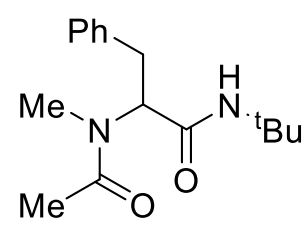

Chemical Formula: $\mathrm{C}_{16} \mathrm{H}_{24} \mathrm{~N}_{2} \mathrm{O}_{2}$

Molecular Weight: 276.38

$N$-(tert-butyl)-2-( $N$-methylacetamido)-3-phenylpropanamide (35) 
Starting from 2-phenylethanol ( $449 \mu \mathrm{L}, 4.50 \mathrm{mmol}$, 3 equiv), methylamine ( $40 \%$ wt in $\mathrm{H}_{2} \mathrm{O}$ ) (143 $\mu \mathrm{L}, 1.65 \mathrm{mmol}, 1.1$ equiv), acetic acid (225 mg, 3.75 mmol, 2.5 equiv) and tert-butyl isocyanide (170 $\mu \mathrm{L}, 1.5 \mathrm{mmol})$, and following the general procedure, product 35 (127 $\mathrm{mg}, 31 \%$ yield) was obtained as a white solid after purification by flash column chromatography. Given the ${ }^{1} \mathrm{H}$ NMR timescale, two rotamers $\left(7: 1\right.$ ratio in $\left.\mathrm{CDCl}_{3}\right)$ were observed. Major rotamer: ${ }^{1} \mathbf{H}$ NMR $\left(300 \mathrm{MHz}, \mathrm{CDCl}_{3}\right): \delta=7.31-7.10(\mathrm{~m}, 5 \mathrm{H})$, 5.86 (br s, 1H), 5.22 (dd, $J=8.5,7.6 \mathrm{~Hz}, 1 \mathrm{H}$ ), 3.24 (dd, $J=14.3,7.6 \mathrm{~Hz}, 1 \mathrm{H}$ ), 2.97-2.87 (m, 4H), $2.02(\mathrm{~s}, 3 \mathrm{H}), 1.27(\mathrm{~s}, 9 \mathrm{H}) \mathrm{ppm} .{ }^{13} \mathbf{C}\left\{{ }^{1} \mathbf{H}\right\}$ NMR $(75 \mathrm{MHz}$, $\left.\mathrm{CDCl}_{3}\right): \delta=171.9,169.4,137.5,129.1,128.6,126.7,57.7,51.3,34.1,31.8$, 28.8, 22.1 ppm. HRMS (ESI+): calcd for $\mathrm{C}_{16} \mathrm{H}_{25} \mathrm{~N}_{2} \mathrm{O}_{2}[\mathrm{M}+\mathrm{H}]^{+}$: 227.1911, found: 227.1911. Characteristic peaks for the minor rotamer: ${ }^{1}$ H NMR (300 $\mathrm{MHz}_{\mathrm{CDCl}}$ ): $\delta=5.54(\mathrm{br} \mathrm{s}, 1 \mathrm{H}), 4.31$ (dd, $\left.J=10.8,3.8 \mathrm{~Hz}, 1 \mathrm{H}\right), 3.50$ (dd, $J=$ 14.4, $3.8 \mathrm{~Hz}, 1 \mathrm{H}), 1.60$ (s, 3H), 1.36 (s, 9H) ppm. ${ }^{13} \mathbf{C}\left\{{ }^{1} \mathbf{H}\right\}$ NMR $(75 \mathrm{MHz}$, $\left.\mathrm{CDCl}_{3}\right): \delta=127.2,28.8 \mathrm{ppm}$.

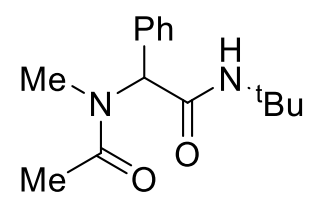

Chemical Formula: $\mathrm{C}_{15} \mathrm{H}_{22} \mathrm{~N}_{2} \mathrm{O}_{2}$

Molecular Weight: 262.35

\section{$N$-(tert-butyl)-2-( $N$-methylacetamido)-2-phenylacetamide (36)}

Starting from benzyl alcohol (466 $\mathrm{L}$, $4.50 \mathrm{mmol}, 3$ equiv), methylamine (40\% wt in $\mathrm{H}_{2} \mathrm{O}$ ) (143 $\mu \mathrm{L}, 1.65 \mathrm{mmol}, 1.1$ equiv), acetic acid (225 mg, $3.75 \mathrm{mmol}$, 2.5 equiv) and tert-butyl isocyanide (170 $\mu \mathrm{L}, 1.5 \mathrm{mmol})$, and following the general procedure, product 36 (81 mg, 20\% yield) was obtained as a white solid after purification by flash column chromatography. ${ }^{1} \mathbf{H}$ NMR $(300 \mathrm{MHz}$, $\left.\mathrm{CDCl}_{3}\right): \delta=7.40-7.30(\mathrm{~m}, 5 \mathrm{H}), 6.19(\mathrm{~s}, 1 \mathrm{H}), 5.52$ (br s, 1H), $2.86(\mathrm{~s}, 3 \mathrm{H}), 2.17$ (s, 3H), 1.36 (s, 9H) ppm. ${ }^{13} \mathbf{C}\left\{{ }^{1} \mathbf{H}\right\}$ NMR $\left(75 \mathrm{MHz}, \mathrm{CDCl}_{3}\right): \delta=171.8,169.1$, 
135.9, 129.3, 128.9, 128.4, 60.4, 51.9, 33.4, 28.8, 22.3 ppm. HRMS (ESI+): calcd for $\mathrm{C}_{15} \mathrm{H}_{22} \mathrm{~N}_{2} \mathrm{O}_{2} \mathrm{Na}[\mathrm{M}+\mathrm{Na}]^{+}:$285.1573, found: 285.1573 .

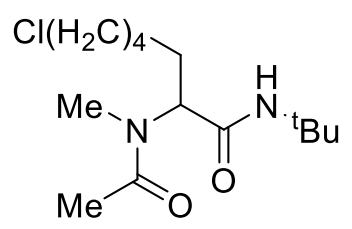

Chemical Formula: $\mathrm{C}_{14} \mathrm{H}_{27} \mathrm{CIN}_{2} \mathrm{O}_{2}$

Molecular Weight: 290.83

\section{$N$-(tert-butyl)-7-chloro-2-( $N$-methylacetamido)heptanamide (37)}

Starting from 6-chlorohexanol ( $600 \mu \mathrm{L}, 4.50 \mathrm{mmol}, 3$ equiv), methylamine (40\% wt in $\mathrm{H}_{2} \mathrm{O}$ ) (143 $\mu \mathrm{L}, 1.65 \mathrm{mmol}, 1.1$ equiv), acetic acid (225 mg, 3.75 mmol, 2.5 equiv) and tert-butyl isocyanide (170 $\mu \mathrm{L}, 1.5 \mathrm{mmol})$, and following the general procedure, product 37 (258 $\mathrm{mg}$, 59\% yield) was obtained as a yellow oil after purification by flash column chromatography. Given the ${ }^{1} \mathrm{H}$ NMR timescale, two rotamers $\left(12: 1\right.$ ratio in $\left.\mathrm{CDCl}_{3}\right)$ were observed. Major rotamer: ${ }^{1} \mathbf{H}$ NMR (300 MHz, $\left.\mathrm{CDCl}_{3}\right): \delta=5.94($ br s, $1 \mathrm{H})$, $4.88(\mathrm{t}, J=7.6 \mathrm{~Hz}, 1 \mathrm{H}), 3.52(\mathrm{t}, J=6.7 \mathrm{~Hz}, 2 \mathrm{H}), 2.91(\mathrm{~s}, 3 \mathrm{H}), 2.13(\mathrm{~s}, 3 \mathrm{H}), 1.95-$ $1.71(\mathrm{~m}, 3 \mathrm{H}), 1.64-1.54(\mathrm{~m}, 2 \mathrm{H}), 1.53-1.41(\mathrm{~m}, 2 \mathrm{H}), 1.30$ (s, 9H), 1.27-1.21 (m, 1H) ppm. ${ }^{13} \mathbf{C}\left\{{ }^{1} \mathbf{H}\right\}$ NMR $\left(75 \mathrm{MHz}, \mathrm{CDCl}_{3}\right): \delta=171.9,169.8,56.5,51.3$, 45.0, 32.5, 31.3, 28.8, 27.4, 26.8, 25.4, 22.2 ppm. HRMS (ESI+): calcd for $\mathrm{C}_{14} \mathrm{H}_{27} \mathrm{ClN}_{2} \mathrm{O}_{2} \mathrm{Na}[\mathrm{M}+\mathrm{Na}]^{+}:$313.1653, found: 313.1653. Characteristic peaks for the minor rotamer: ${ }^{1} \mathrm{H}$ NMR $\left(300 \mathrm{MHz}, \mathrm{CDCl}_{3}\right): \delta=5.55$ (br s, $\left.1 \mathrm{H}\right), 3.42$ $3.34(\mathrm{~m}, 2 \mathrm{H}), 2.79(\mathrm{~s}, 3 \mathrm{H}), 2.15(\mathrm{~s}, 3 \mathrm{H}), 1.34(\mathrm{~s}, 9 \mathrm{H}) \mathrm{ppm}$.

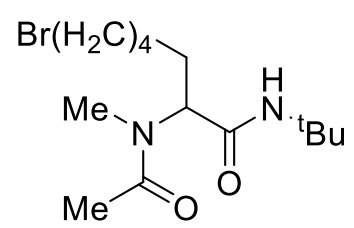

Chemical Formula: $\mathrm{C}_{14} \mathrm{H}_{27} \mathrm{BrN}_{2} \mathrm{O}_{2}$ Molecular Weight: 335.29 
Starting from 6-bromohexanol ( $589 \mu \mathrm{L}, 4.50 \mathrm{mmol}$, 3 equiv), methylamine ( $40 \%$ wt in $\mathrm{H}_{2} \mathrm{O}$ ) (143 $\mu \mathrm{L}, 1.65 \mathrm{mmol}, 1.1$ equiv), acetic acid (225 mg, 3.75 mmol, 2.5 equiv) and tert-butyl isocyanide (170 $\mu \mathrm{L}, 1.5 \mathrm{mmol})$, and following the general procedure, product 38 (220 mg, 44\% yield) was obtained as a colorless oil after purification by flash column chromatography. Given the ${ }^{1} \mathrm{H}$ NMR timescale, two rotamers (14:1 ratio in $\mathrm{CDCl}_{3}$ ) were observed. Major rotamer: ${ }^{\mathbf{1}} \mathbf{H}$ NMR (300 MHz, $\mathrm{CDCl}_{3}$ ): $\delta=5.95$ (br s, 1H), $4.88(\mathrm{t}, J=7.7 \mathrm{~Hz}, 1 \mathrm{H}), 3.39$ (t, J = 6.8 Hz, 2H), $2.90(\mathrm{~s}, 3 \mathrm{H}), 2.13(\mathrm{~s}$, $3 \mathrm{H}), 1.93-1.79(\mathrm{~m}, 3 \mathrm{H}), 1.67-1.40(\mathrm{~m}, 4 \mathrm{H}), 1.30(\mathrm{~s}, 9 \mathrm{H}), 1.27-1.20(\mathrm{~m}, 1 \mathrm{H})$ ppm. ${ }^{13} \mathbf{C}\left\{{ }^{1} \mathrm{H}\right\}$ NMR $\left(75 \mathrm{MHz}, \mathrm{CDCl}_{3}\right): \delta=171.9,169.8,56.5,51.3,33.8,32.6$, 31.3, 28.8, 28.0, 27.4, 25.3, 22.2 ppm. HRMS (EI+): calcd for $\mathrm{C}_{14} \mathrm{H}_{28} \mathrm{BrN}_{2} \mathrm{O}_{2}$ $[\mathrm{M}+\mathrm{H}]^{+}:$335.1329, found: 335.1329. Characteristic peaks for the minor rotamer: ${ }^{1} \mathrm{H}$ NMR $\left(300 \mathrm{MHz}, \mathrm{CDCl}_{3}\right): \delta=2.79(\mathrm{~s}, 3 \mathrm{H}), 1.34(\mathrm{~s}, 9 \mathrm{H}) \mathrm{ppm}$.

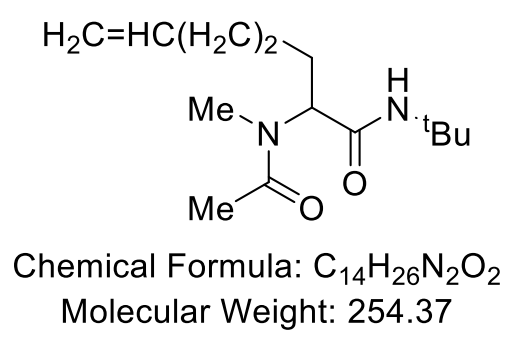

\section{$N$-(tert-butyl)-2-( $N$-methylacetamido)hept-6-enamide (39)}

Starting from 5-hexen-1-ol (540 $\mu \mathrm{L}, 4.50 \mathrm{mmol}, 3$ equiv), methylamine (40\% wt in $\mathrm{H}_{2} \mathrm{O}$ ) (143 $\mu \mathrm{L}, 1.65 \mathrm{mmol}, 1.1$ equiv), acetic acid (225 mg, $3.75 \mathrm{mmol}$, 2.5 equiv) and tert-butyl isocyanide (170 $\mu \mathrm{L}, 1.5 \mathrm{mmol})$, and following the general procedure, product 39 (246 $\mathrm{mg}$, 64\% yield) was obtained as a yellow oil after purification by flash column chromatography. Given the ${ }^{1} \mathrm{H}$ NMR timescale, two rotamers (11:1 ratio in $\left.\mathrm{CDCl}_{3}\right)$ were observed. $\underline{\text { Major }}$ rotamer: ${ }^{1} \mathbf{H}$ NMR $\left(300 \mathrm{MHz}, \mathrm{CDCl}_{3}\right): \delta=5.94(\mathrm{br} \mathrm{s}, 1 \mathrm{H}), 5.77(\mathrm{ddt}, J=16.9$, 
10.2, $6.7 \mathrm{~Hz}, 1 \mathrm{H}), 5.08-4.81(\mathrm{~m}, 3 \mathrm{H}), 2.90(\mathrm{~s}, 3 \mathrm{H}), 2.13$ (s, 3H), 2.11-1.99 (m, 2H), 1.94-1.78 (m, 1H), 1.67-1.53 (m, 2H), 1.43-1.22 (m, 1H), 1.30 (s, 9H) ppm. ${ }^{13} \mathbf{C}\left\{{ }^{1} \mathbf{H}\right\}$ NMR (75 MHz, $\left.\mathrm{CDCl}_{3}\right): \delta=171.9,169.9,138.3,115.1,56.5$, 51.2, 33.5, 31.3, 28.8, 27.0, 25.4, 22.2 ppm. HRMS (EI+): calcd for $\mathrm{C}_{14} \mathrm{H}_{27} \mathrm{~N}_{2} \mathrm{O}_{2}[\mathrm{M}+\mathrm{H}]^{+}:$255.2067, found: 255.2055. Characteristic peaks for the minor rotamer: ${ }^{1} \mathbf{H}$ NMR $\left(300 \mathrm{MHz}, \mathrm{CDCl}_{3}\right): \delta=5.55$ (br s, $\left.1 \mathrm{H}\right), 4.17-4.03$ (m, 2H), 2.79 (s, 3H), 2.15 (s, 3H) ppm. ${ }^{13} \mathbf{C}\left\{{ }^{1} \mathrm{H}\right\}$ NMR (75 MHz, $\left.\mathrm{CDCl}_{3}\right): \delta=60.5$, $51.8,28.0 \mathrm{ppm}$.

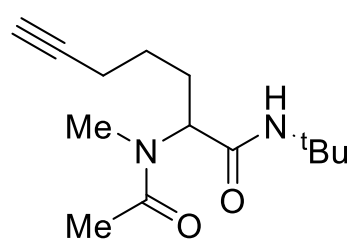

Chemical Formula: $\mathrm{C}_{14} \mathrm{H}_{24} \mathrm{~N}_{2} \mathrm{O}_{2}$

Molecular Weight: 252.36

\section{$N$-(tert-butyl)-2-(N-methylacetamido)hept-6-ynamide (40)}

Starting from 5-hexyn-1-ol 0 (496 $\mu \mathrm{L}, 4.50 \mathrm{mmol}, 3$ equiv), methylamine (40\% wt in $\mathrm{H}_{2} \mathrm{O}$ ) 0 (143 $\mu \mathrm{L}, 1.65 \mathrm{mmol}, 1.1$ equiv), acetic acid () (225 mg, $3.75 \mathrm{mmol}, 2.5$ equiv) and tert-butyl isocyanide $0(170 \mu \mathrm{L}, 1.5 \mathrm{mmol})$, and following the general procedure, product 40 (152 $\mathrm{mg}, 40 \%$ yield) was obtained as a yellowish solid after purification by flash column chromatography. Given the ${ }^{1} \mathrm{H}$ NMR timescale, two rotamers (10:1 ratio in $\mathrm{CDCl}_{3}$ ) were observed. Major rotamer: ${ }^{1} \mathbf{H}$ NMR $\left(300 \mathrm{MHz}, \mathrm{CDCl}_{3}\right): \delta=5.96$ (br s, 1H), 4.89 (dd, $J=8.2,7.2 \mathrm{~Hz}, 1 \mathrm{H}), 2.89$ (s, 3H), 2.25-2.14 (m, 2H), 2.11 (s, 3H), 1.98-1.86 (m, 2H), 1.80-1.64 (m, 1H), 1.53-1.34 (m, 2H), $1.27(\mathrm{~s}, 9 \mathrm{H})$ ppm. ${ }^{13} \mathbf{C}\left\{{ }^{1} \mathrm{H}\right\}$ NMR $\left(75 \mathrm{MHz}, \mathrm{CDCl}_{3}\right): \delta=171.9,169.6,83.9,68.9,56.0,51.2$, 31.2, 28.7, 26.6, 25.0, 22.1, 18.3 ppm. HRMS (ESI+): calcd for $\mathrm{C}_{14} \mathrm{H}_{24} \mathrm{~N}_{2} \mathrm{O}_{2} \mathrm{Na}$ [M+Na]': 275.1730, found: 275.1730. Characteristic peaks for the minor rotamer: ${ }^{1} \mathbf{H}$ NMR $\left(300 \mathrm{MHz}, \mathrm{CDCl}_{3}\right): \delta=5.61(\mathrm{br} \mathrm{s}, 1 \mathrm{H}), 2.78(\mathrm{~s}, 3 \mathrm{H}), 1.31(\mathrm{~s}$, 
9H) ppm. ${ }^{13} \mathbf{C}\left\{{ }^{1} \mathbf{H}\right\}$ NMR $\left(75 \mathrm{MHz}, \mathrm{CDCl}_{3}\right): \delta=171.5,168.7,83.4,69.3,62.3$, $51.7,29.7,27.8,25.4,21.9 \mathrm{ppm}$.

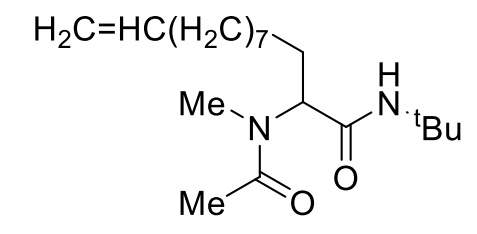

Chemical Formula: $\mathrm{C}_{19} \mathrm{H}_{36} \mathrm{~N}_{2} \mathrm{O}_{2}$

Molecular Weight: 324.51

\section{$N$-(tert-butyl)-2-(N-methylacetamido)dodec-11-enamide (41)}

Starting from 10-undecen-1-ol (902 $\mu \mathrm{L}, 4.50 \mathrm{mmol}, 3$ equiv), methylamine (40\% wt in $\mathrm{H}_{2} \mathrm{O}$ ) (143 $\mu \mathrm{L}, 1.65 \mathrm{mmol}, 1.1$ equiv), acetic acid (225 mg, 3.75 mmol, 2.5 equiv) and tert-butyl isocyanide (170 $\mu \mathrm{L}, 1.5 \mathrm{mmol})$, and following the general procedure, product 41 (256 mg, 53\% yield) was obtained as a yellow oil after purification by flash column chromatography. Given the ${ }^{1} \mathrm{H}$ NMR timescale, two rotamers $\left(10: 1\right.$ ratio in $\left.\mathrm{CDCl}_{3}\right)$ were observed. Major rotamer: ${ }^{1} \mathbf{H}$ NMR (300 MHz, $\mathrm{CDCl}_{3}$ ): $\delta=5.97$ (br s, $\left.1 \mathrm{H}\right)$, 5.87-5.68 (m, 1H), 5.03-4.77 (m, 3H), 2.88 (s, 3H), 2.09 (s, 3H), 2.04-1.95 (m, 2H), $1.88-1.71(\mathrm{~m}, 1 \mathrm{H}), 1.63-1.46(\mathrm{~m}, 1 \mathrm{H}), 1.37-1.15(\mathrm{~m}, 21 \mathrm{H}) \mathrm{ppm} .{ }^{13} \mathbf{C}\left\{{ }^{1} \mathrm{H}\right\}$ NMR $\left(75 \mathrm{MHz}, \mathrm{CDCl}_{3}\right): \delta=171.8,170.0,139.2,114.2,56.6,51.1,33.8,31.2$, 29.42, 29.39 (2C), 29.1, 28.9, 28.7, 27.5, 26.0, 22.1 ppm. HRMS (EI+): calcd for $\mathrm{C}_{19} \mathrm{H}_{37} \mathrm{~N}_{2} \mathrm{O}_{2}[\mathrm{M}+\mathrm{H}]^{+}:$325.2850, found: 325.2851. Characteristic peaks for the minor rotamer: ${ }^{1} \mathrm{H}$ NMR $\left(300 \mathrm{MHz}, \mathrm{CDCl}_{3}\right): \delta=5.61$ (br s, $\left.1 \mathrm{H}\right), 4.13-4.00$ (m, 2H), 2.76 (s, 3H) ppm. ${ }^{13} \mathbf{C}\left\{{ }^{1} \mathbf{H}\right\}$ NMR (75 MHz, $\left.\mathrm{CDCl}_{3}\right): \delta=171.6,169.1$, $133.2,62.7,51.6,26.4 \mathrm{ppm}$. 


\section{Gram scale reaction}<smiles>CC(=O)N(CC(=O)NC1CCCCC1)Cc1ccccc1</smiles>

Chemical Formula: $\mathrm{C}_{17} \mathrm{H}_{24} \mathrm{~N}_{2} \mathrm{O}_{2}$

Molecular Weight: 288.3910

\section{$N$-benzyl- $N$-(2-(cyclohexylamino)-2-oxoethyl)acetamide ${ }^{1}(6)$}

Starting from $791 \mu \mathrm{L}, 15.0 \mathrm{mmol}, 3$ equiv), benzylamine ( $981 \mu \mathrm{L}, 5.5 \mathrm{mmol}$, 1.1 equiv), acetic acid (1.05 mL, $12.5 \mathrm{mmol}, 2.5$ equiv) and cyclohexyl isocyanide (878 $\mu \mathrm{L}, 5.0 \mathrm{mmol}$ ), and following the general procedure, product 6 (1.08 g, 75\% yield) was obtained as a white solid after purification by flash column chromatography. 
VI. NMR Spectra of all multicomponent adducts.

${ }^{1} \mathrm{H}$ NMR of 5 in $\mathrm{CDCl}_{3}$

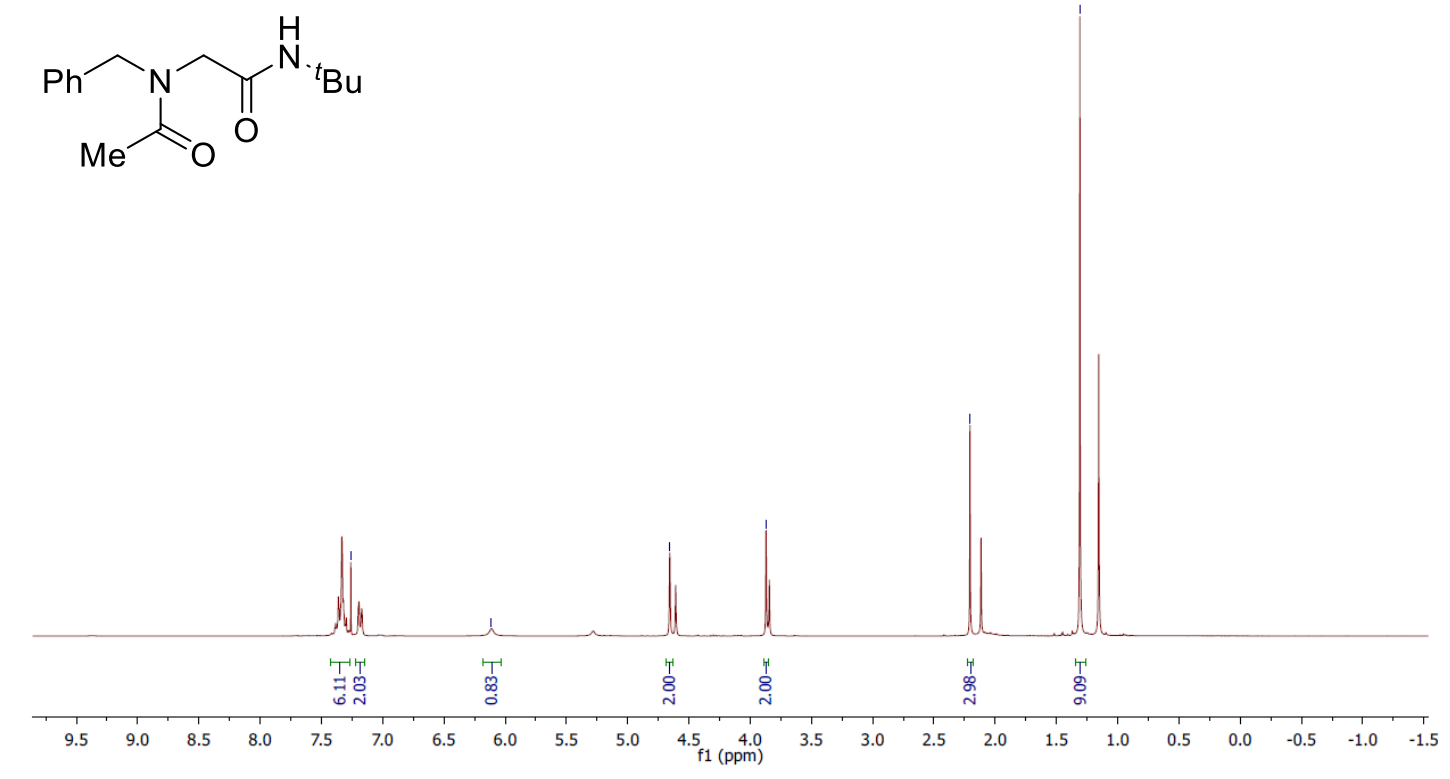

${ }^{13} \mathrm{C}\left\{{ }^{1} \mathrm{H}\right\}$ NMR of 5 in $\mathrm{CDCl}_{3}$

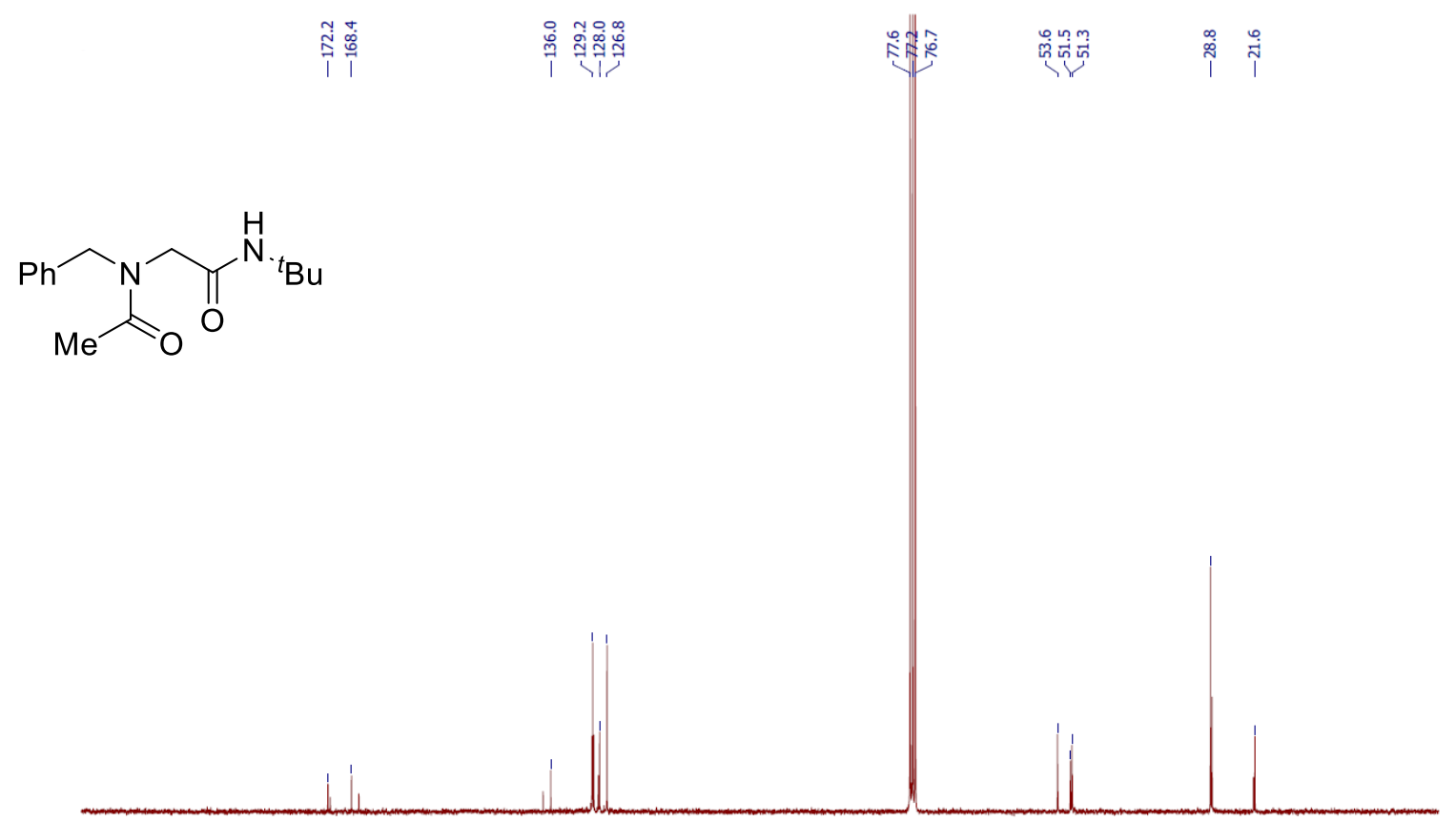

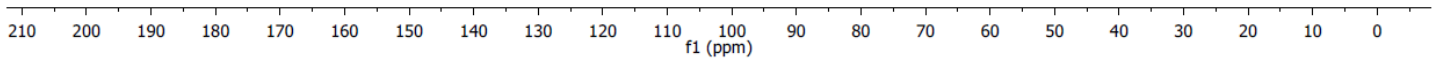


${ }^{1} \mathrm{H} \mathrm{NMR}$ of 6 in $\mathrm{CDCl}_{3}$

i<smiles>CNC(=O)CN(Cc1ccccc1)C(C)=O</smiles>

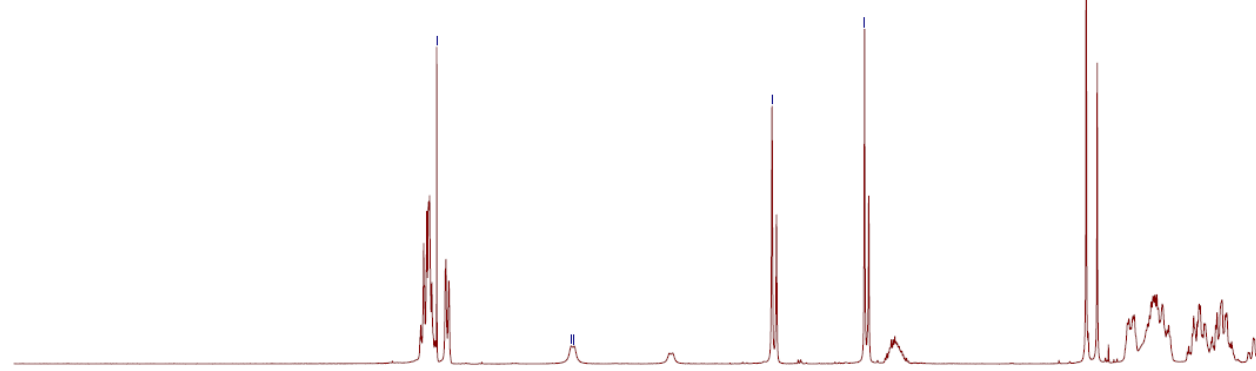

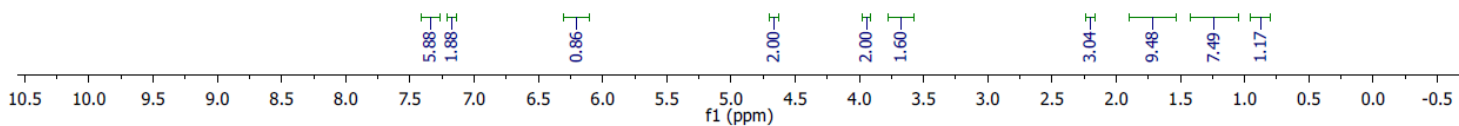

${ }^{13} \mathrm{C}\left\{{ }^{1} \mathrm{H}\right\} \mathrm{NMR}$ of 6 in $\mathrm{CDCl}_{3}$

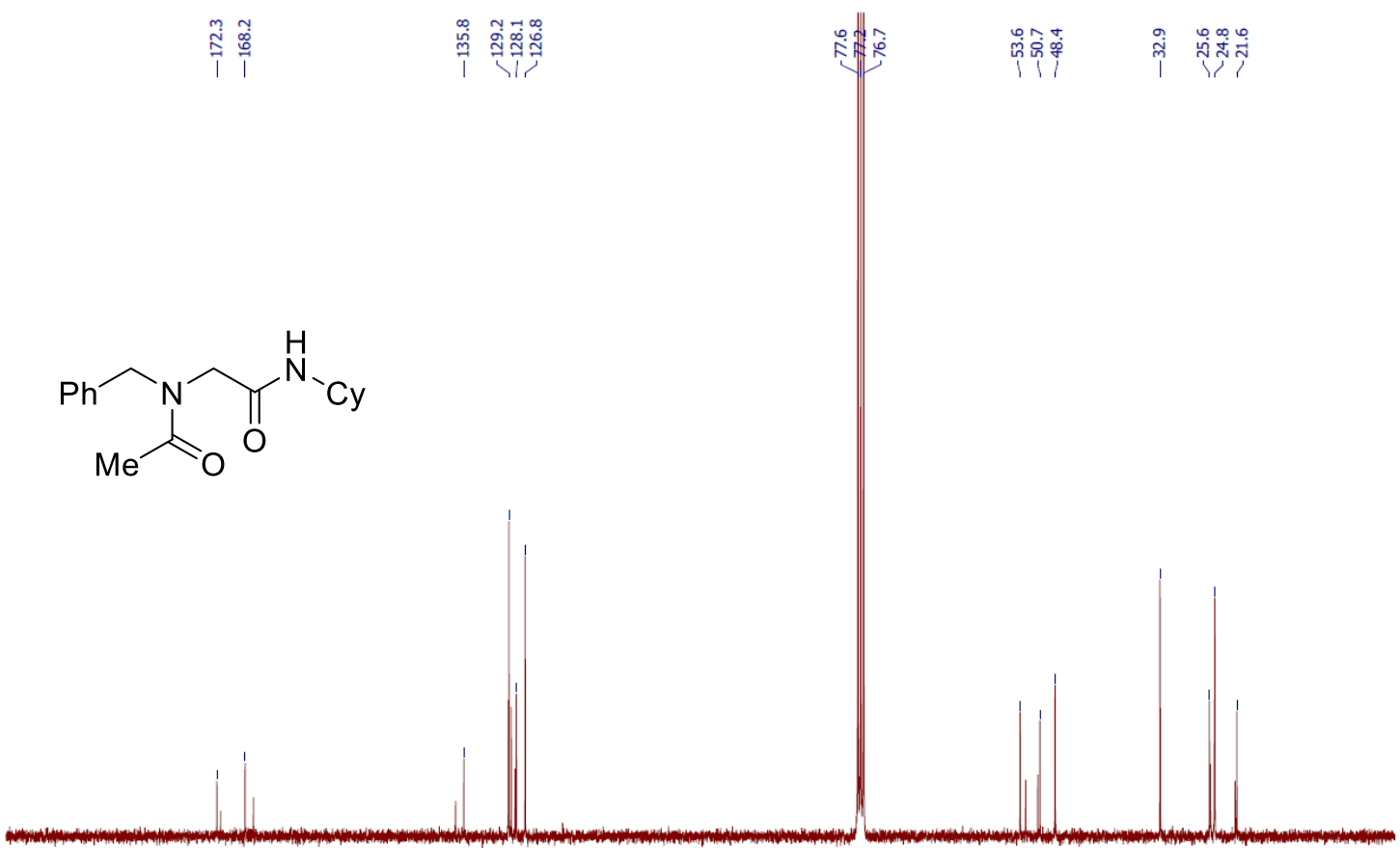

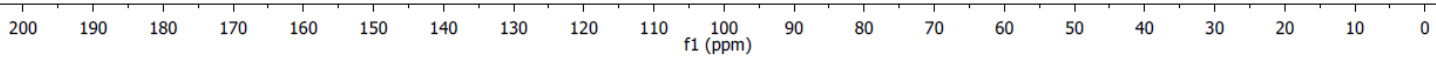


${ }^{1} \mathrm{H}$ NMR of 7 in $\mathrm{CDCl}_{3}$

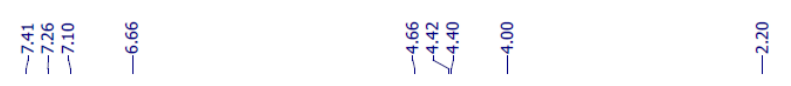<smiles>CC(=O)N(CC(=O)NCc1ccccc1)Cc1ccccc1</smiles>

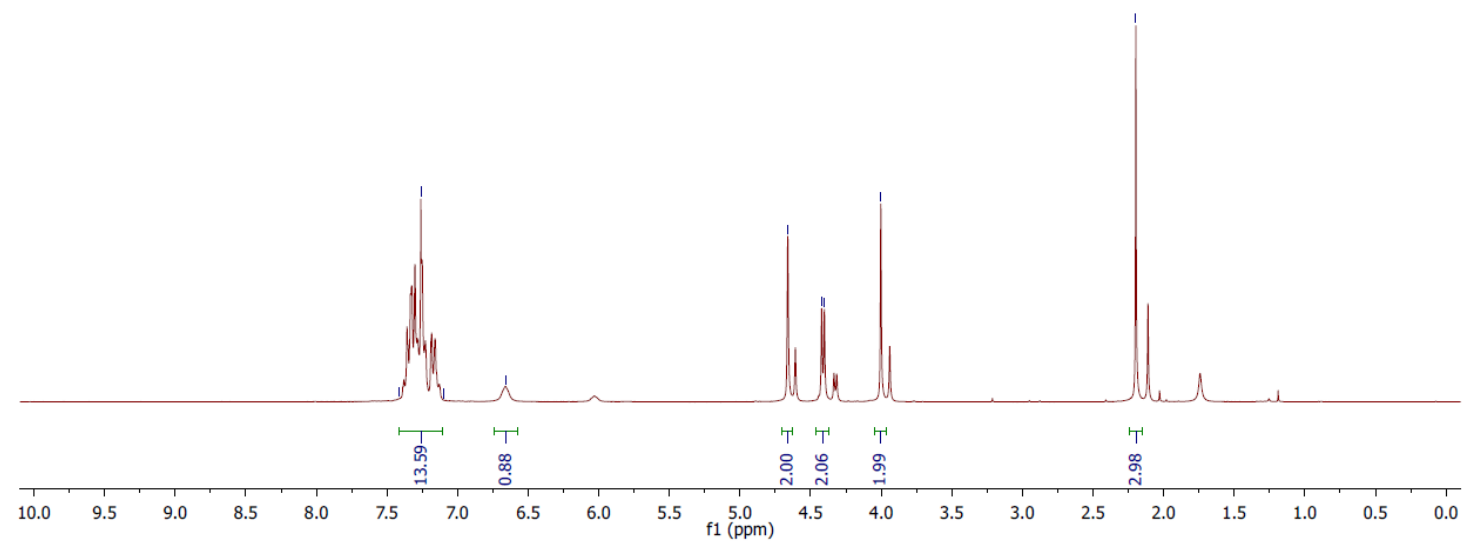

${ }^{13} \mathrm{C}\left\{{ }^{1} \mathrm{H}\right\}$ NMR of 7 in $\mathrm{CDCl}_{3}$

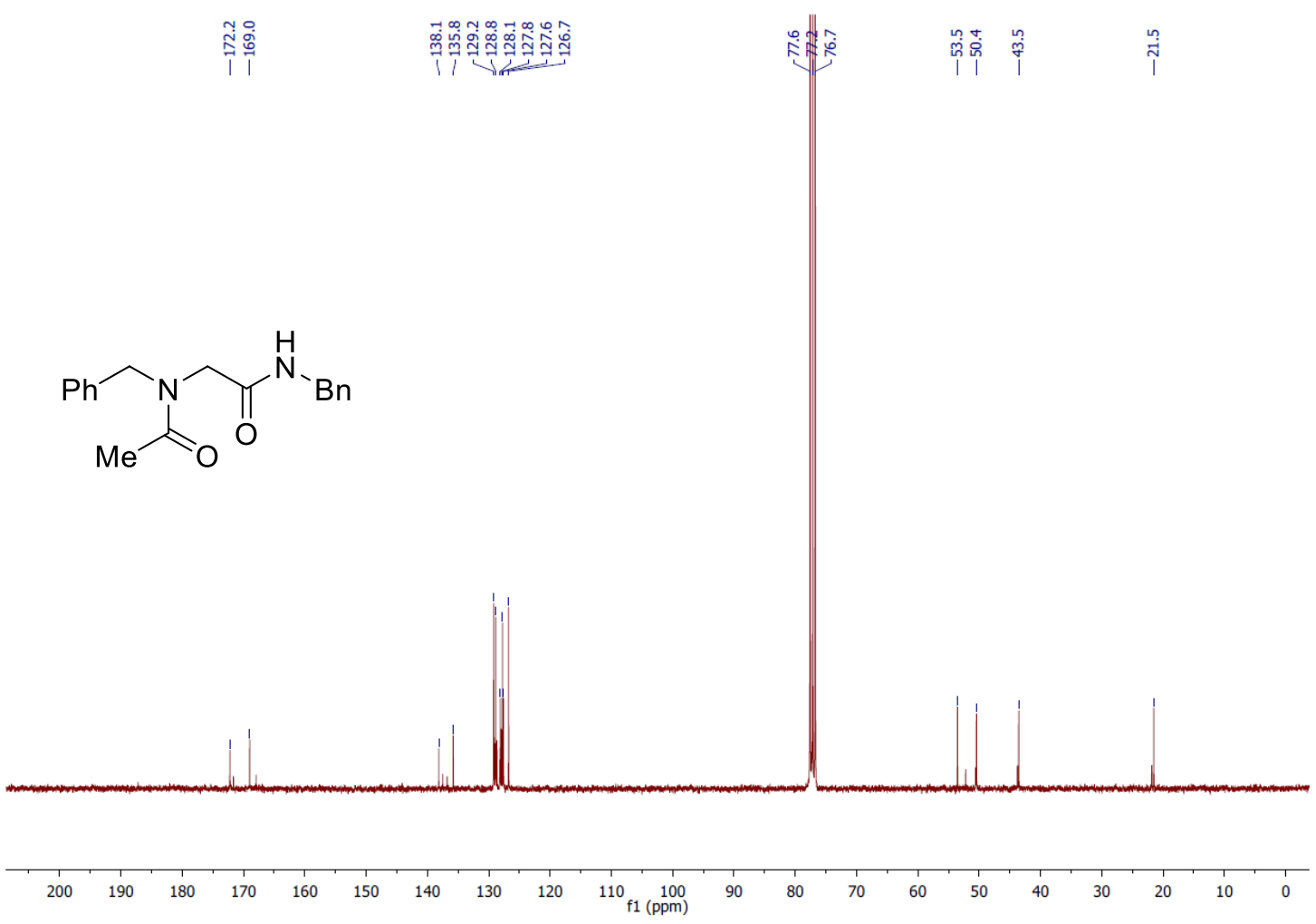


${ }^{1} \mathrm{H}$ NMR of 8 in $\mathrm{CDCl}_{3}$

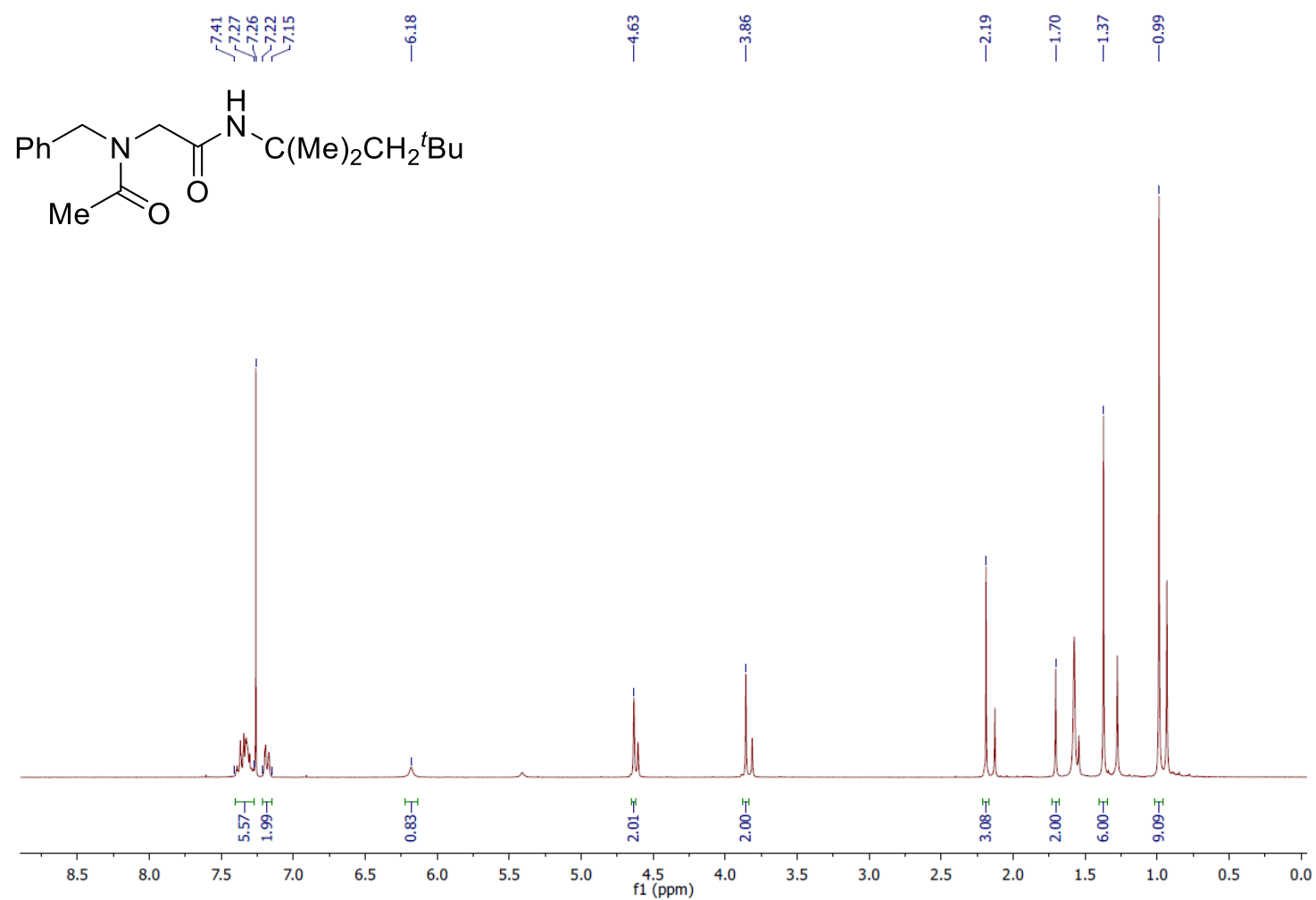

${ }^{13} \mathrm{C}\left\{{ }^{1} \mathrm{H}\right\} \mathrm{NMR}$ of 8 in $\mathrm{CDCl}_{3}$

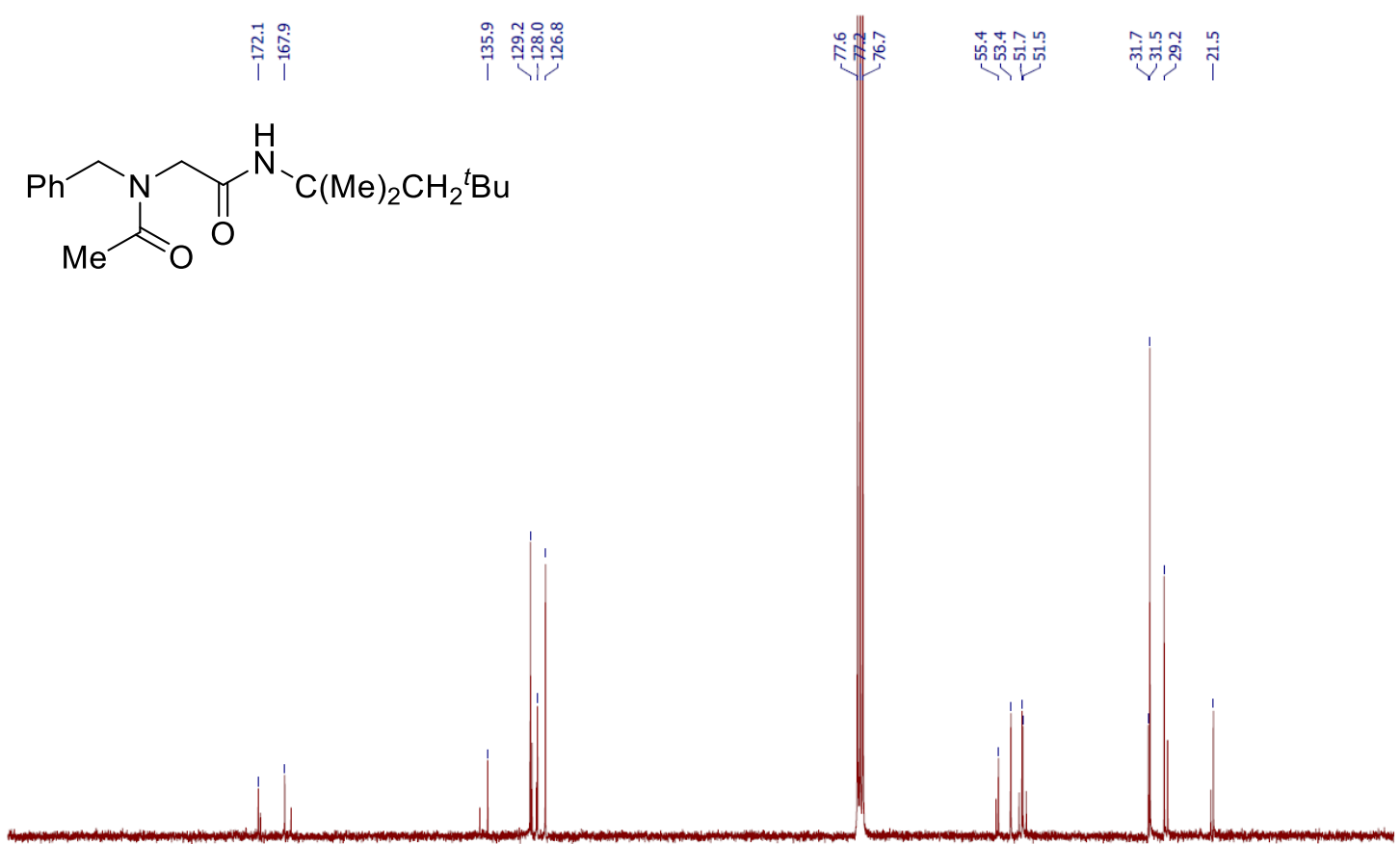

$\begin{array}{llllllllllllllllllllllllll}210 & 200 & 190 & 180 & 170 & 160 & 150 & 140 & 130 & 120 & 110 & 100 & 90 & 80 & 70 & 60 & 50 & 40 & 30 & 20 & 10 & 0\end{array}$ 
${ }^{1} \mathrm{H}$ NMR of 9 in $\mathrm{CDCl}_{3}$

$$
\text { 穷 }
$$<smiles>CC(=O)CNC(=O)CN(Cc1ccccc1)C(C)=O</smiles>

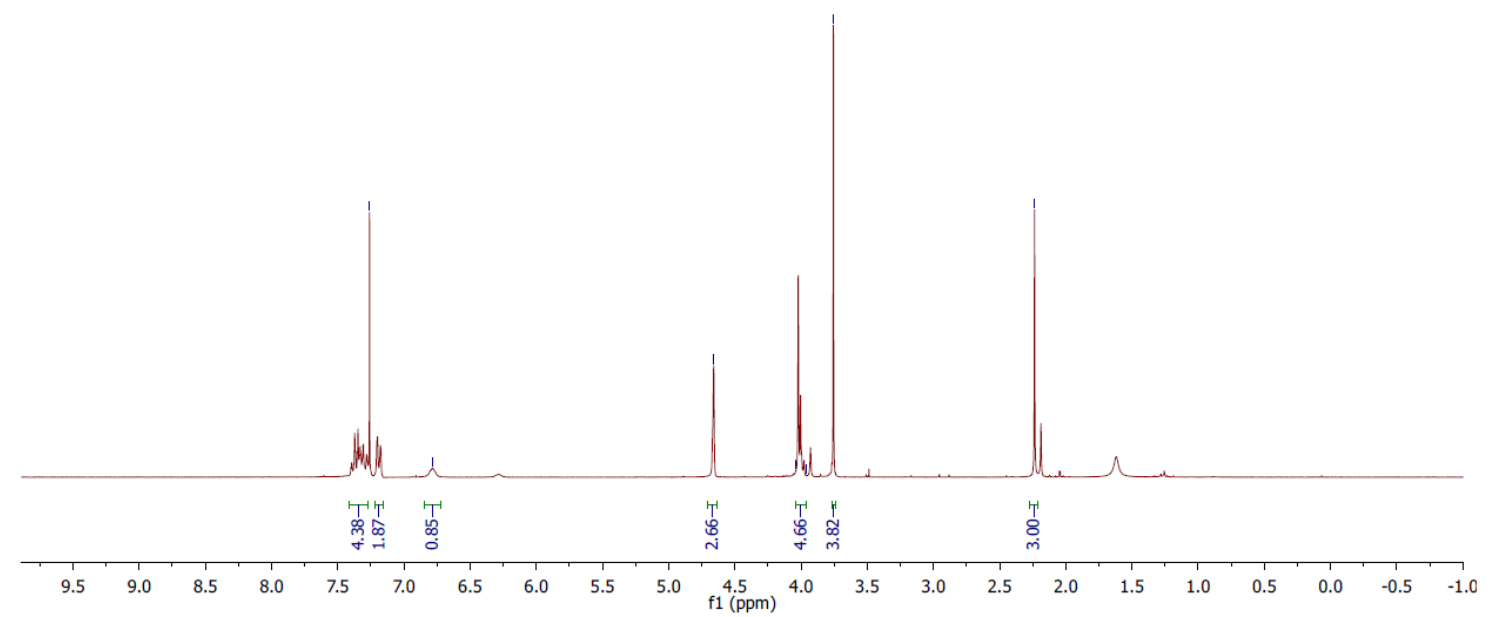

${ }^{13} \mathrm{C}\left\{{ }^{1} \mathrm{H}\right\} \mathrm{NMR}$ of 9 in $\mathrm{CDCl}_{3}$
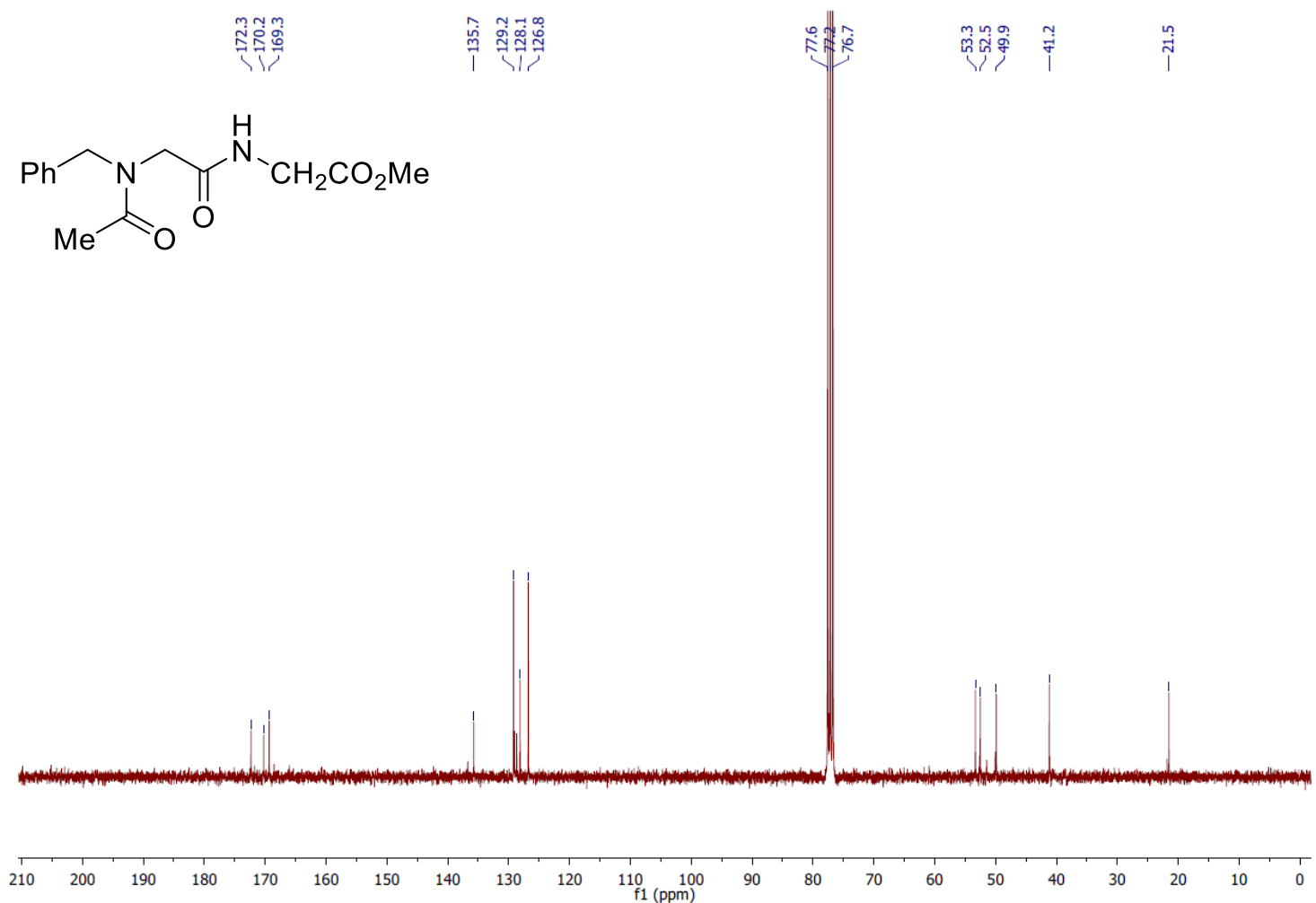
${ }^{1} \mathrm{H}$ NMR of 10 in $\mathrm{CDCl}_{3}$
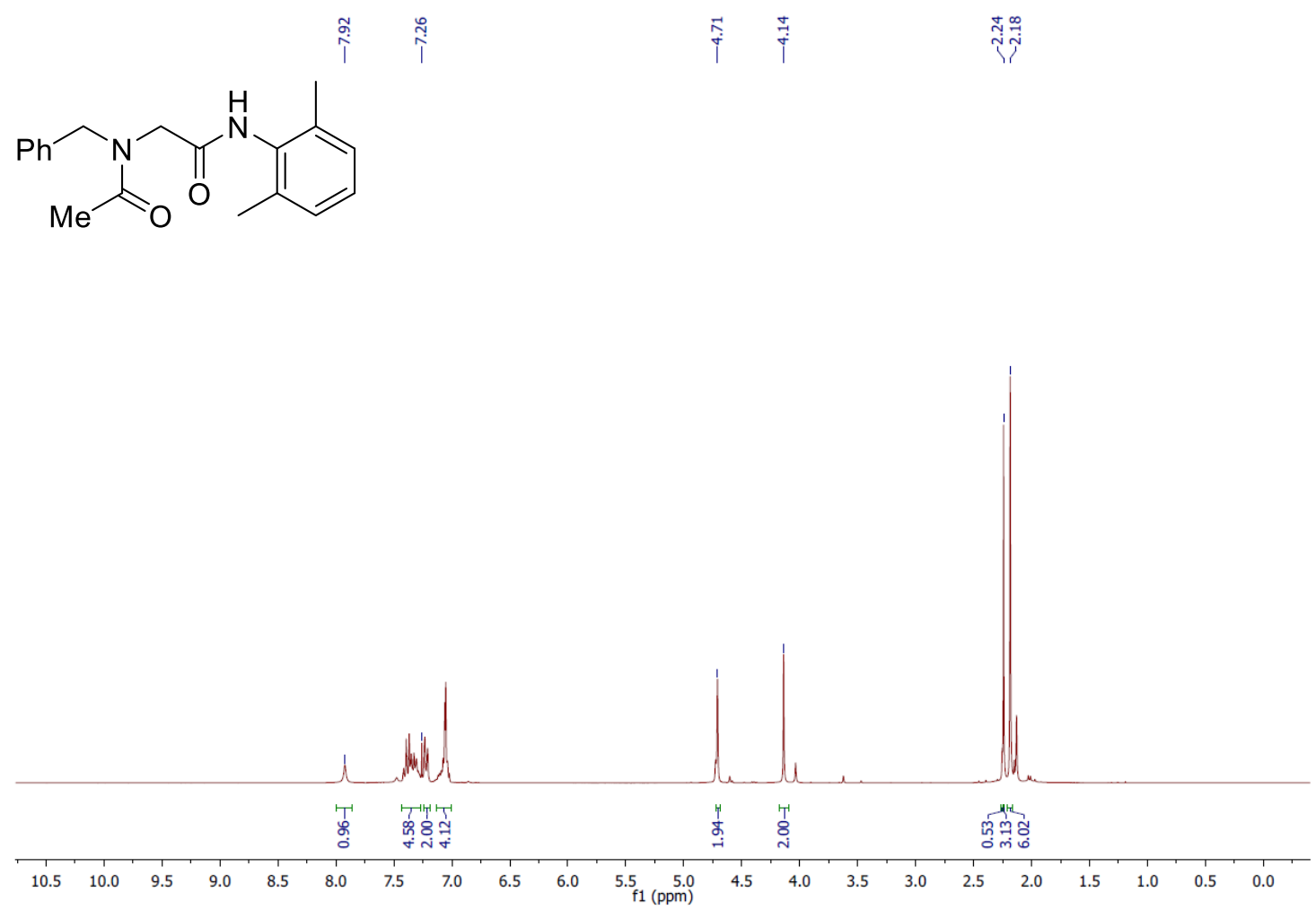

${ }^{13} \mathrm{C}\left\{{ }^{1} \mathrm{H}\right\} \mathrm{NMR}$ of $\mathbf{1 0}$ in $\mathrm{CDCl}_{3}$
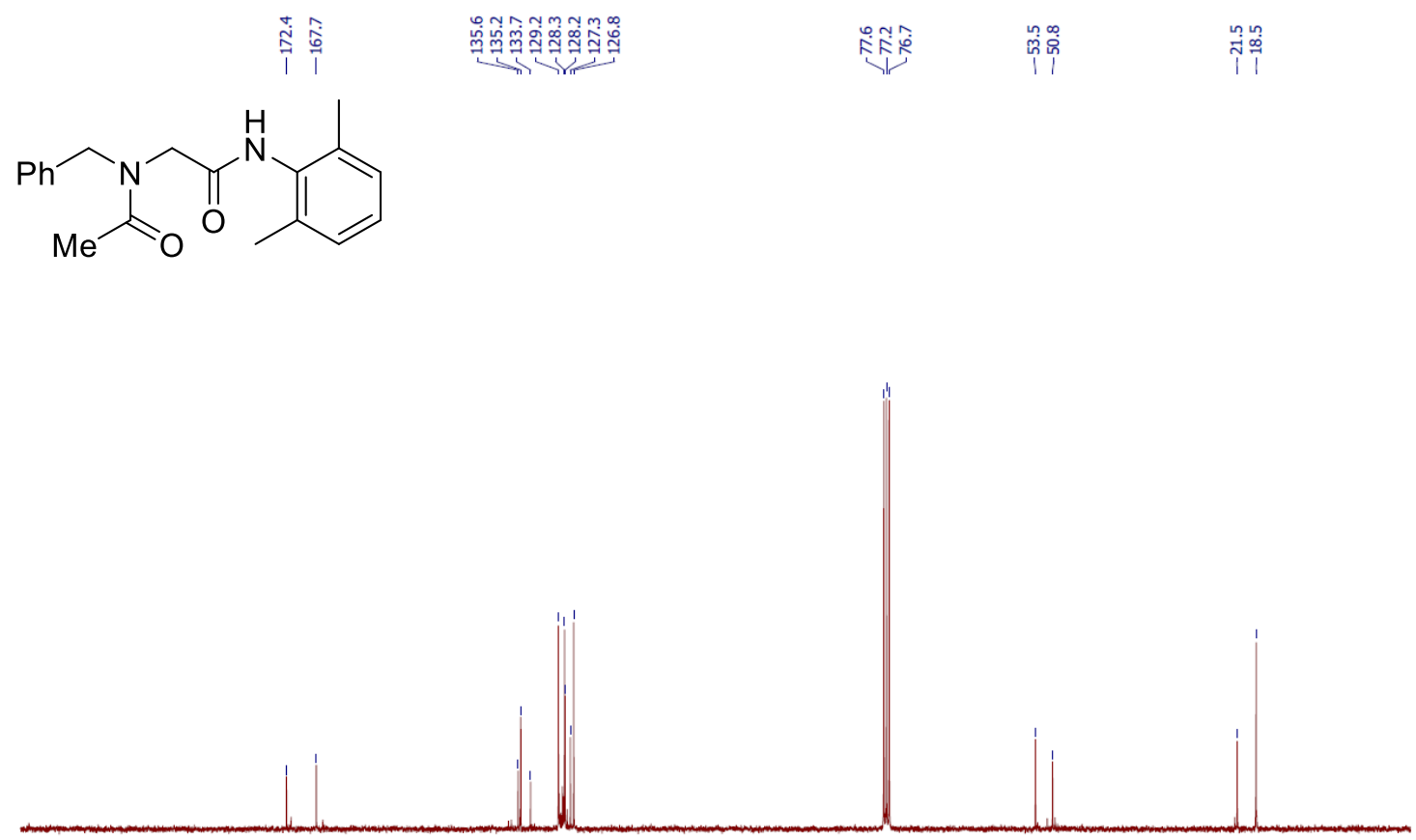

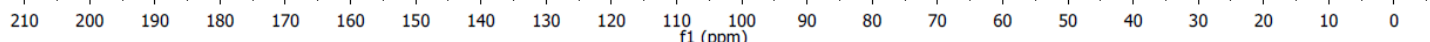


${ }^{1} \mathrm{H}$ NMR of 11 in $\mathrm{CDCl}_{3}$

i<smiles>CC(=O)N(C)CC(=O)NC(C)(C)C</smiles>

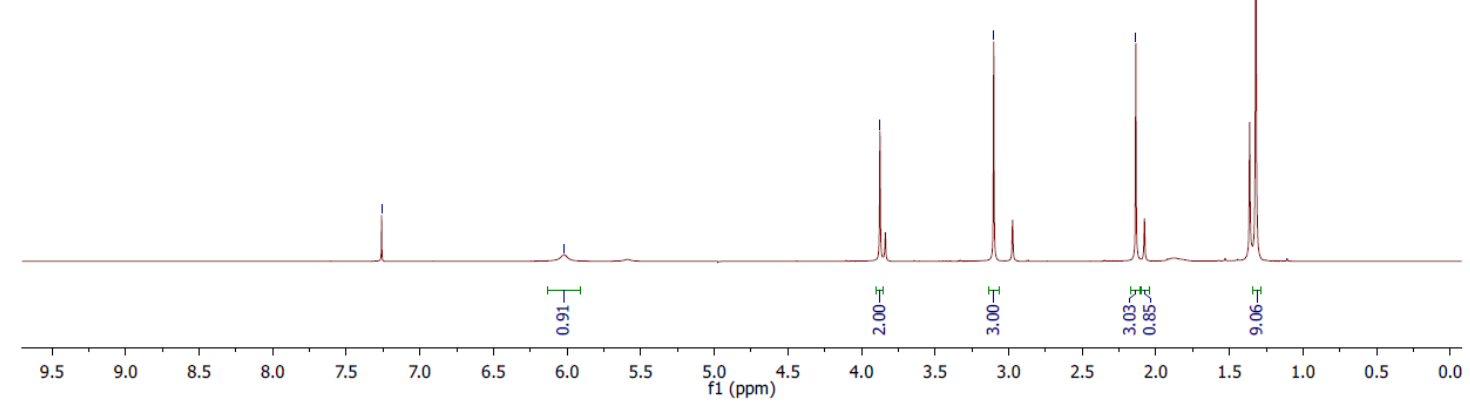

${ }^{13} \mathrm{C}\left\{{ }^{1} \mathrm{H}\right\}$ NMR of 11 in $\mathrm{CDCl}_{3}$
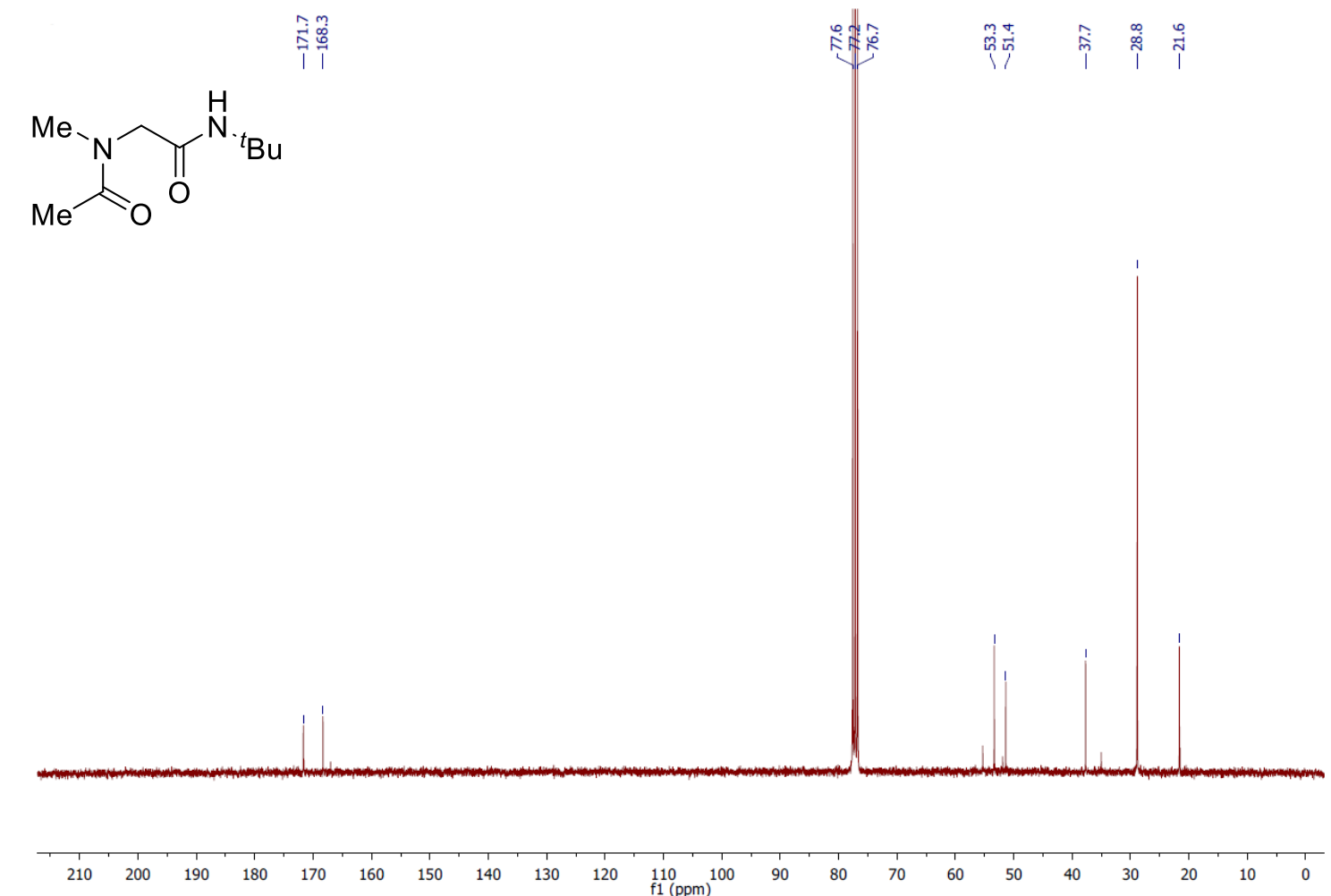
${ }^{1} \mathrm{H}$ NMR of 12 in $\mathrm{CDCl}_{3}$

i<smiles>CC(=O)N(CC(=O)NC(C)(C)C)C(C)(C)C</smiles>

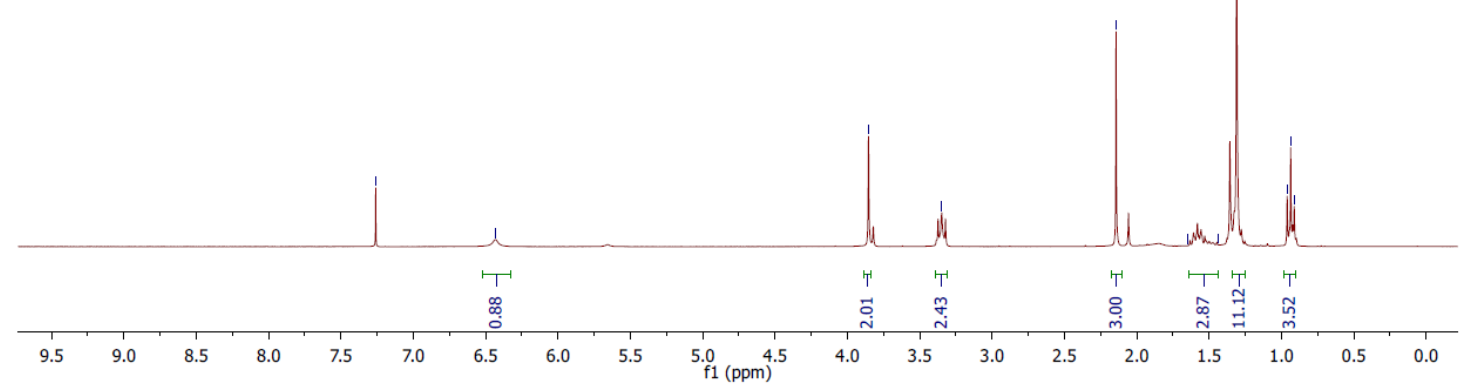

${ }^{13} \mathrm{C}\left\{{ }^{1} \mathrm{H}\right\}$ NMR of 12 in $\mathrm{CDCl}_{3}$

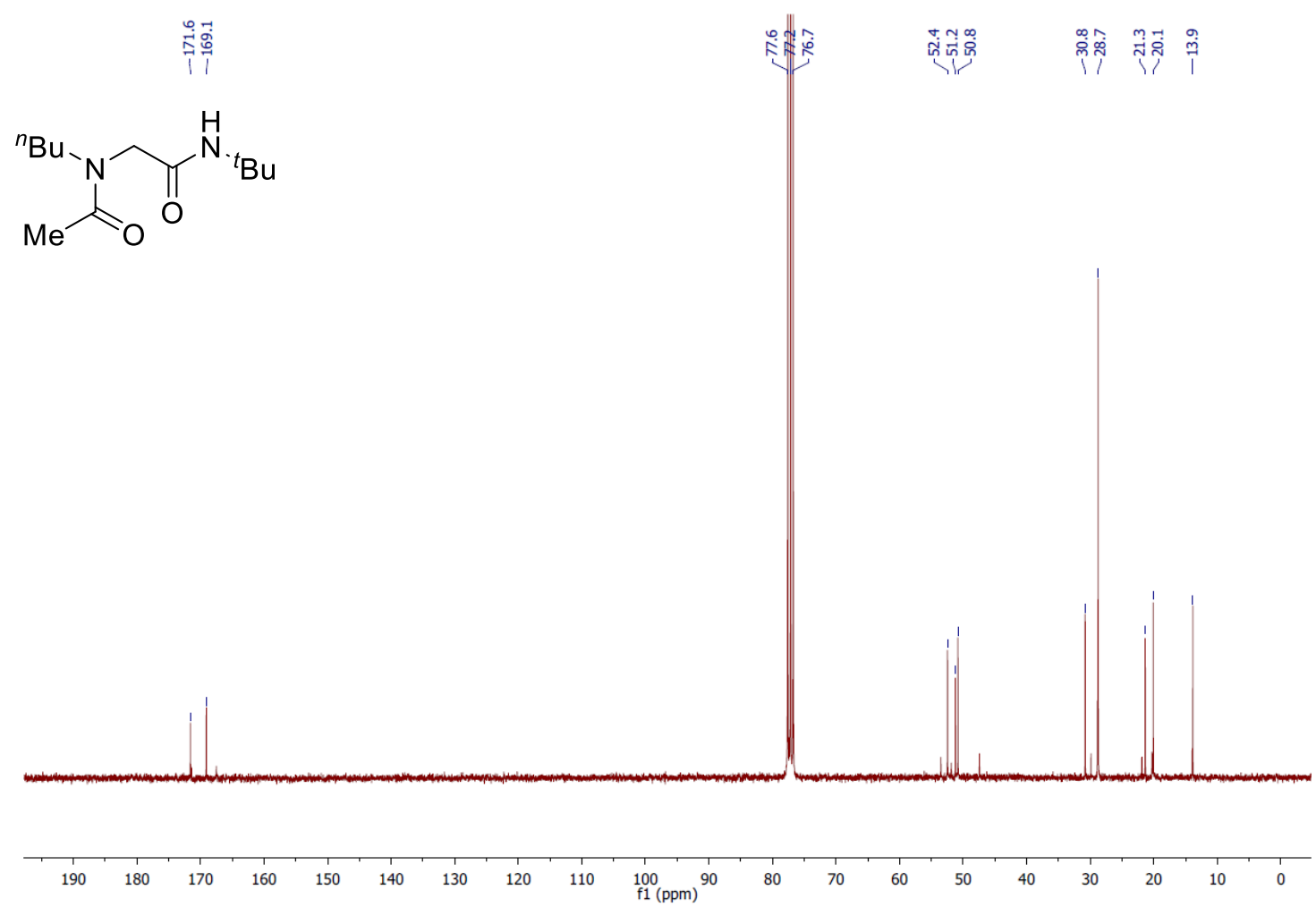


${ }^{1} \mathrm{H}$ NMR of 13 in $\mathrm{CDCl}_{3}$

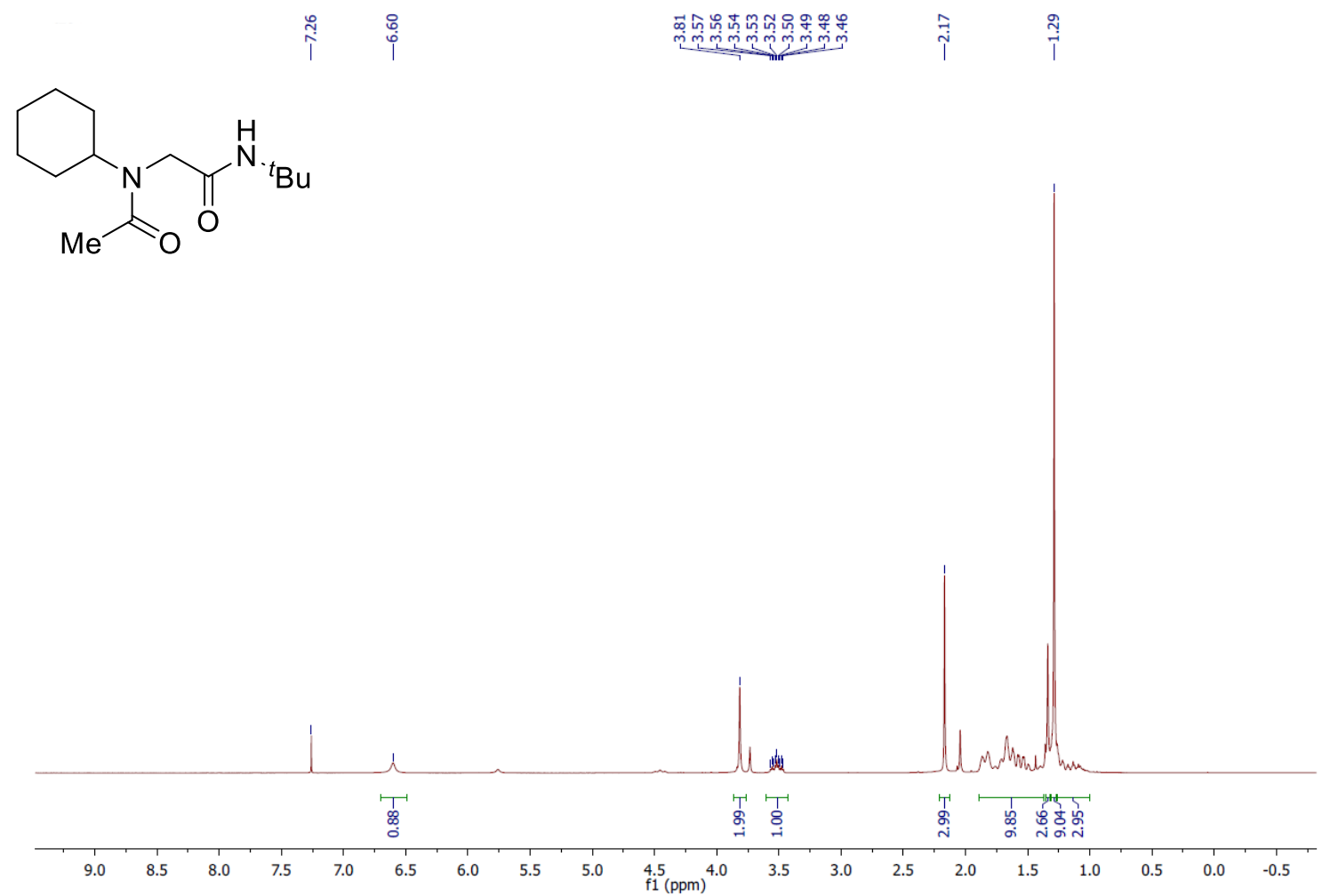

${ }^{13} \mathrm{C}\left\{{ }^{1} \mathrm{H}\right\} \mathrm{NMR}$ of $\mathbf{1 3}$ in $\mathrm{CDCl}_{3}$
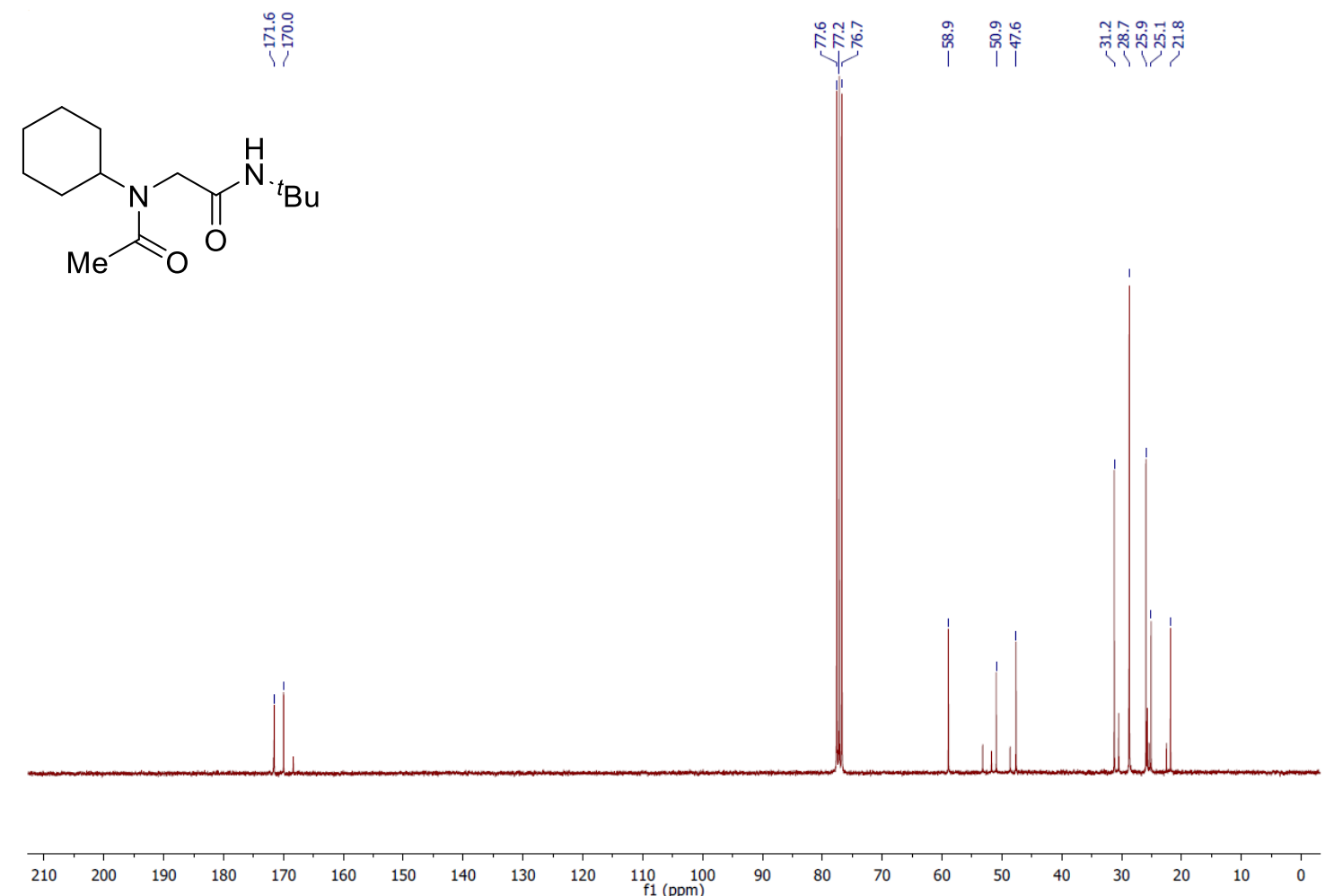
${ }^{1} \mathrm{H}$ NMR of 14 in $\mathrm{CDCl}_{3}$

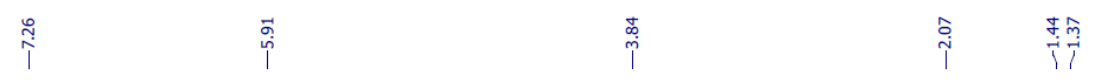<smiles>CC(=O)N(CC(=O)NC(C)(C)C)CC(C)(C)C</smiles>

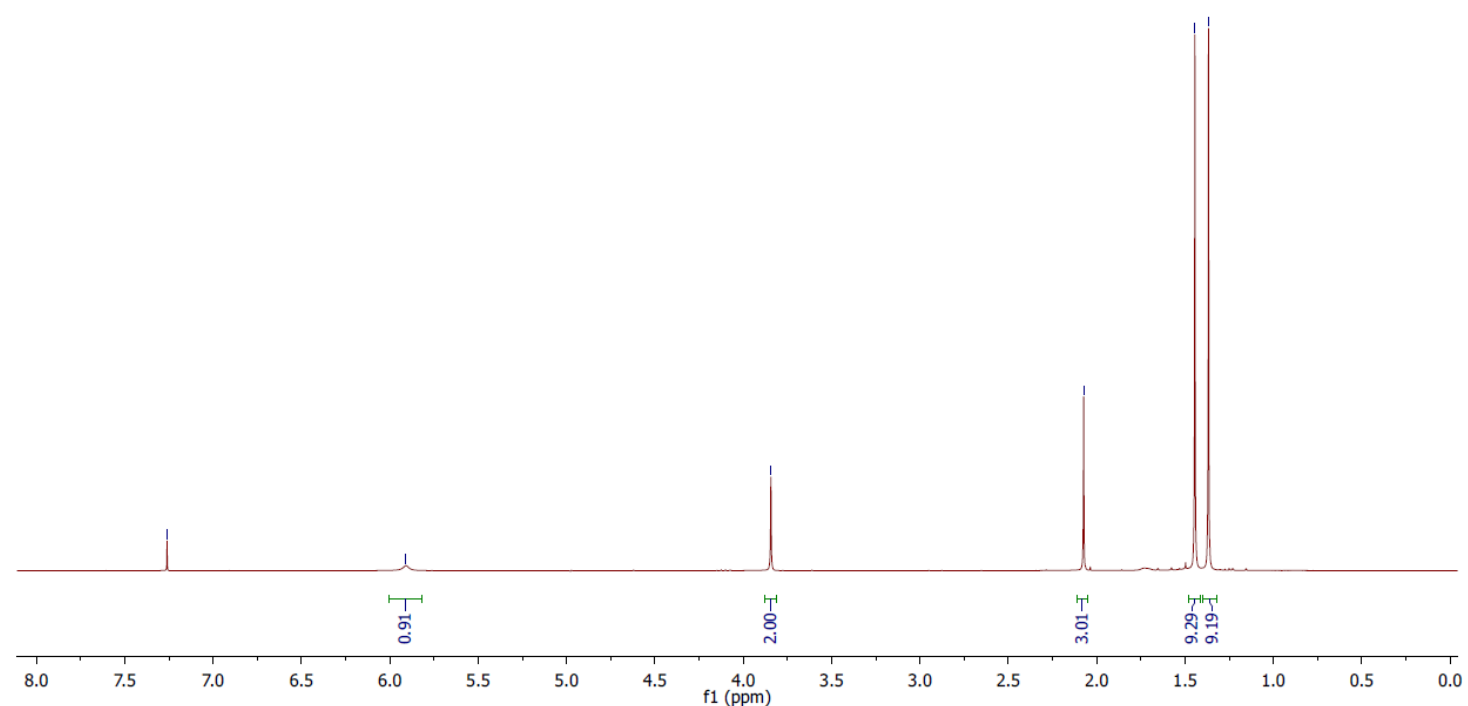

${ }^{13} \mathrm{C}\left\{{ }^{1} \mathrm{H}\right\} \mathrm{NMR}$ of 14 in $\mathrm{CDCl}_{3}$<smiles>CCCCNC(=O)CN(CC(C)(C)C)C(C)=O</smiles> 
${ }^{1} \mathrm{H}$ NMR of 15 in $\mathrm{CDCl}_{3}$
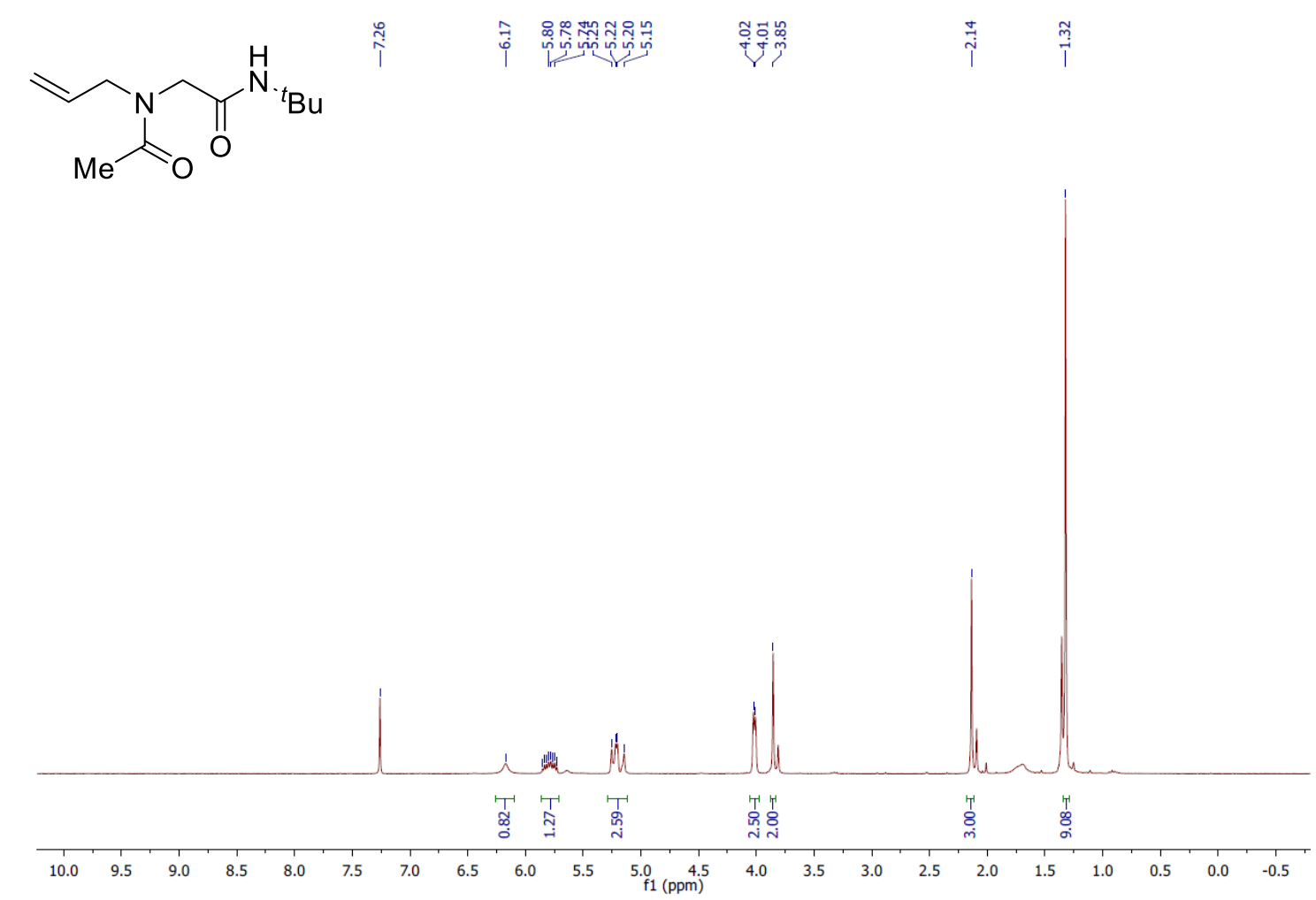

${ }^{13} \mathrm{C}\left\{{ }^{1} \mathrm{H}\right\} \mathrm{NMR}$ of 15 in $\mathrm{CDCl}_{3}$

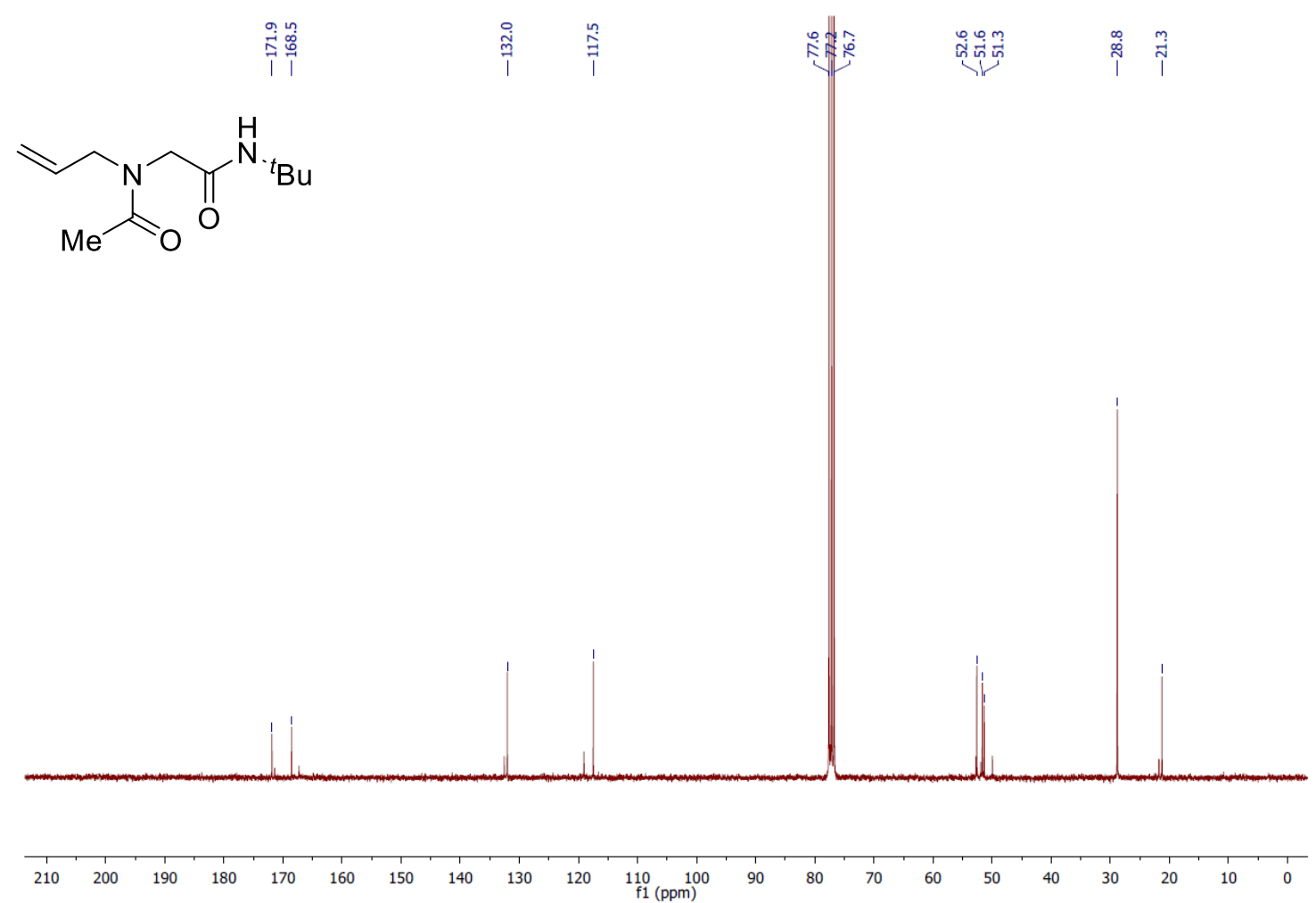


${ }^{1} \mathrm{H}$ NMR of 16 in $\mathrm{CDCl}_{3}$

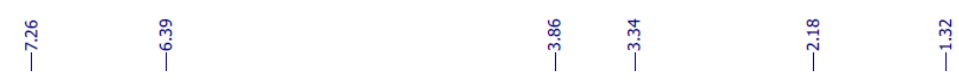<smiles>COCCN(CC(=O)NC(C)(C)C)C(C)=O</smiles>

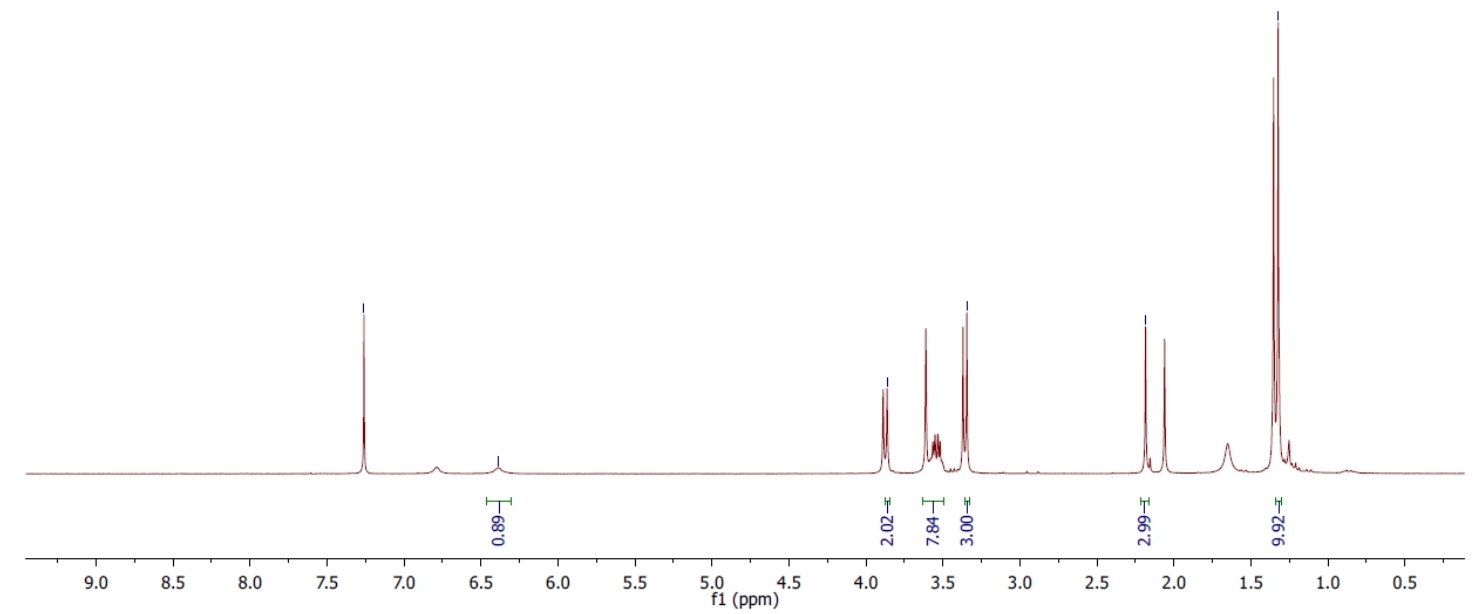

${ }^{13} \mathrm{C}\left\{{ }^{1} \mathrm{H}\right\}$ NMR of $\mathbf{1 6}$ in $\mathrm{CDCl}_{3}$<smiles>CCCCNC(=O)CN(CCOC)C(C)=O</smiles>

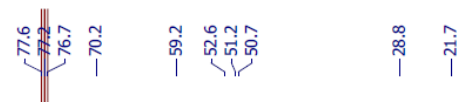

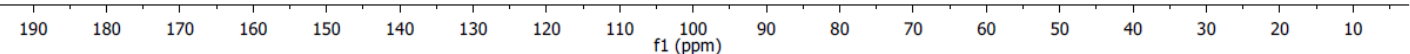


${ }^{1} \mathrm{H}$ NMR of 17 in $\mathrm{CDCl}_{3}$

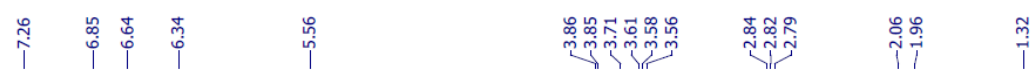<smiles>COc1ccc(CCN(CC(=O)NC(C)(C)C)C(C)=O)cc1OC</smiles>

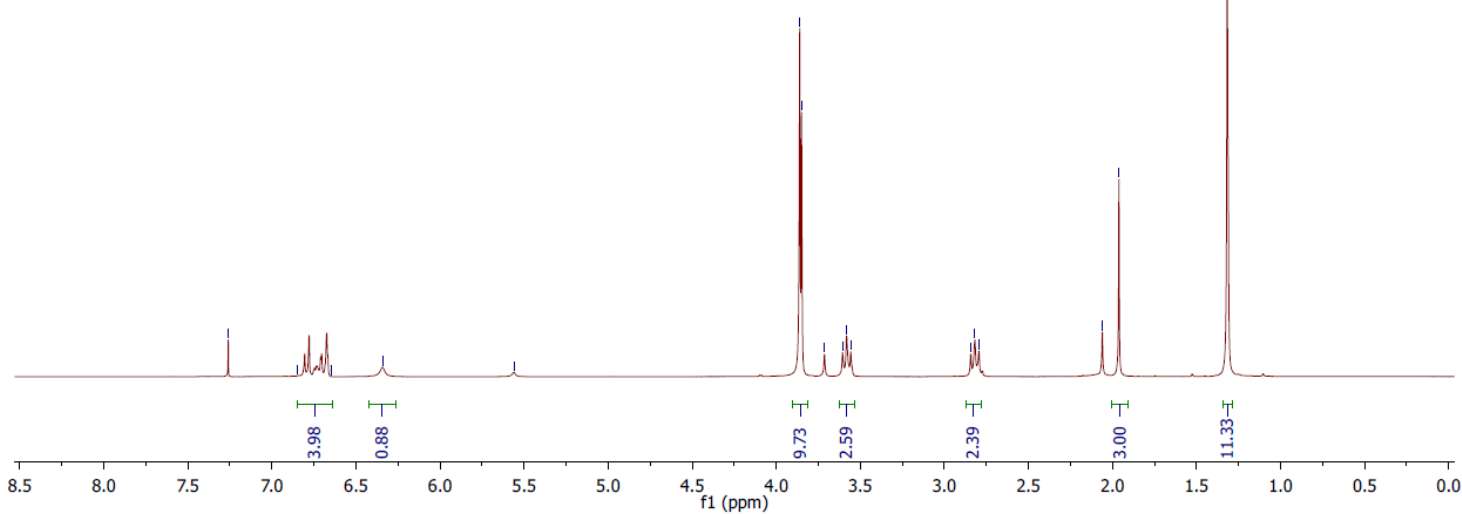

${ }^{13} \mathrm{C}\left\{{ }^{1} \mathrm{H}\right\}$ NMR of $\mathbf{1 7}$ in $\mathrm{CDCl}_{3}$

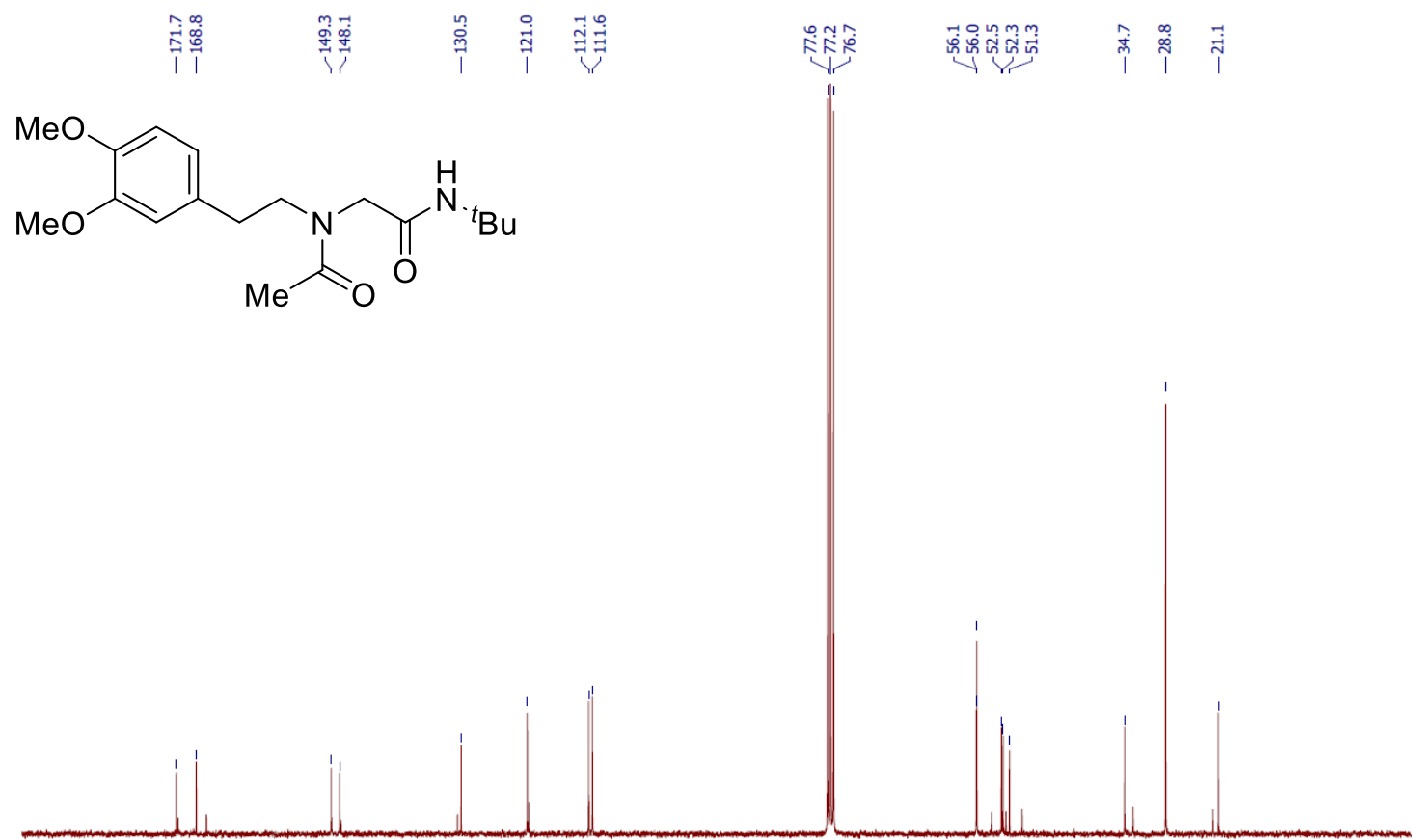

$\begin{array}{llllllllll}190 & 180 & 170 & 160 & 150 & 140 & 130 & 120 & 110 & 100 \begin{array}{r}90 \\ \mathrm{f} 1(\mathrm{ppm})\end{array}\end{array}$ 
${ }^{1} \mathrm{H}$ NMR of 18 in $\mathrm{CDCl}_{3}$

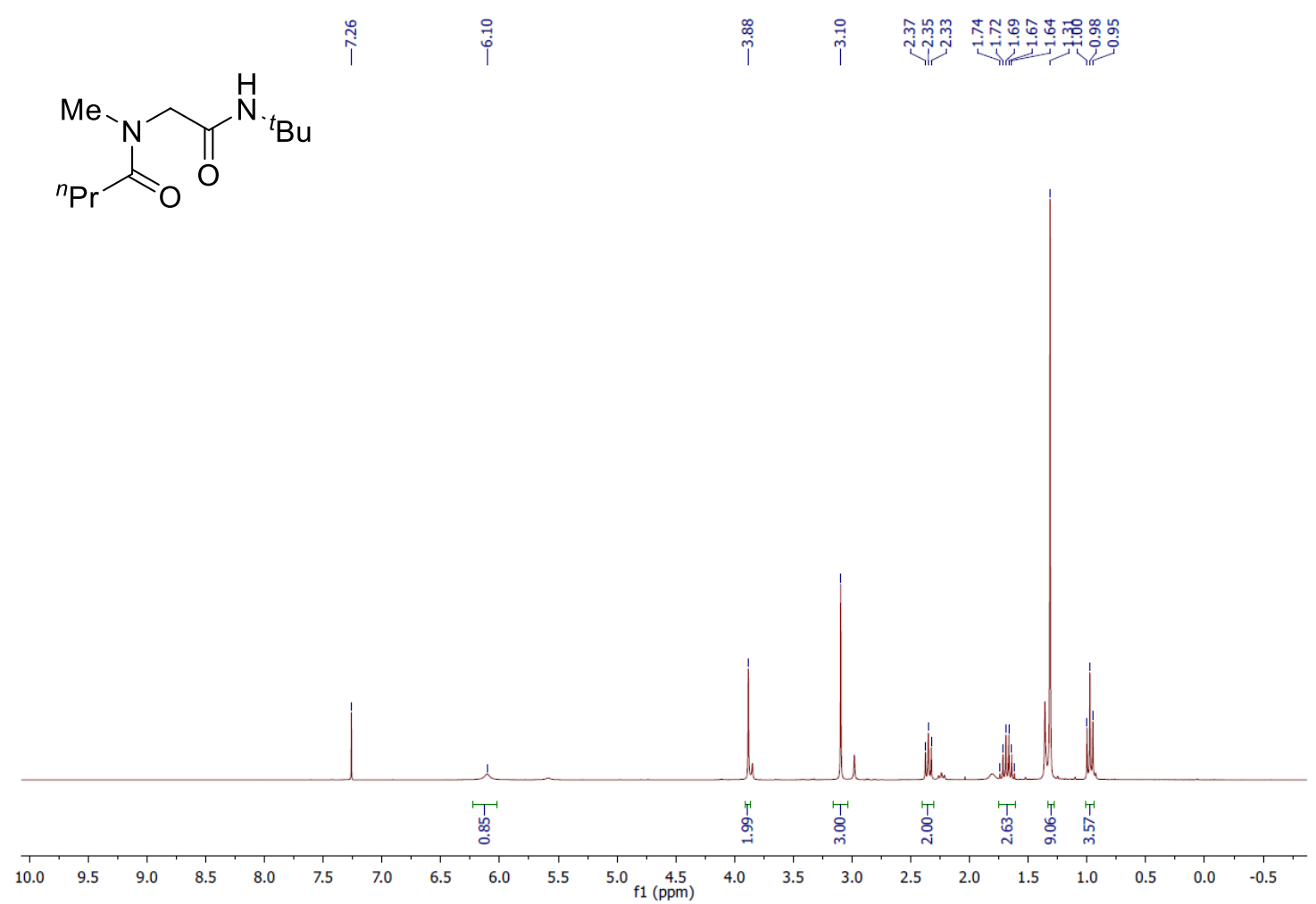

${ }^{13} \mathrm{C}\left\{{ }^{1} \mathrm{H}\right\} \mathrm{NMR}$ of 18 in $\mathrm{CDCl}_{3}$

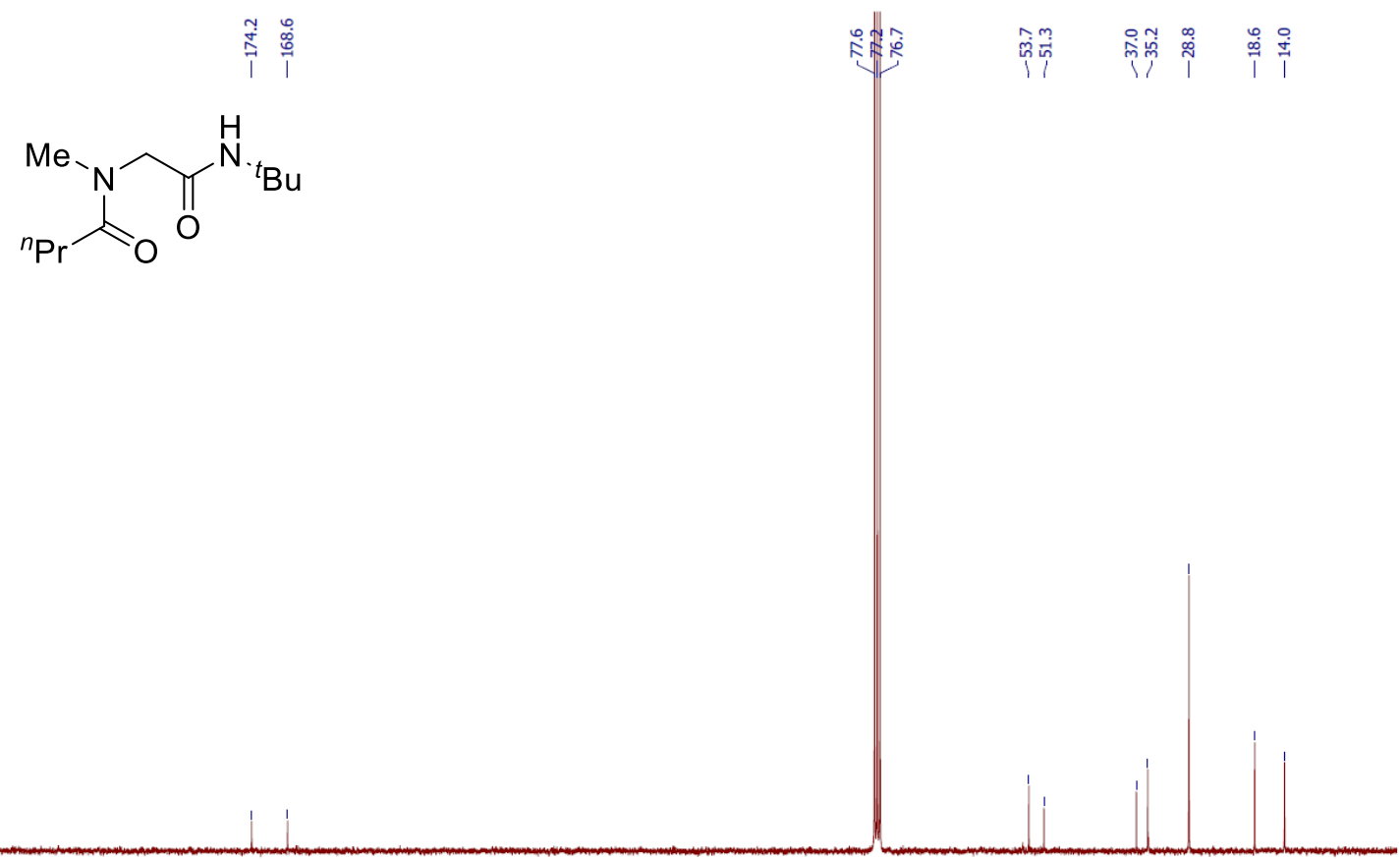

$\begin{array}{lllllllllllllllllllllllll}210 & 200 & 190 & 180 & 170 & 160 & 150 & 140 & 130 & 120 & 110 & 100 & 90 & 80 & 70 & 60 & 50 & 40 & 30 & 20 & 10 & 0\end{array}$ 
${ }^{1} \mathrm{H}$ NMR of 19 in $\mathrm{CDCl}_{3}$

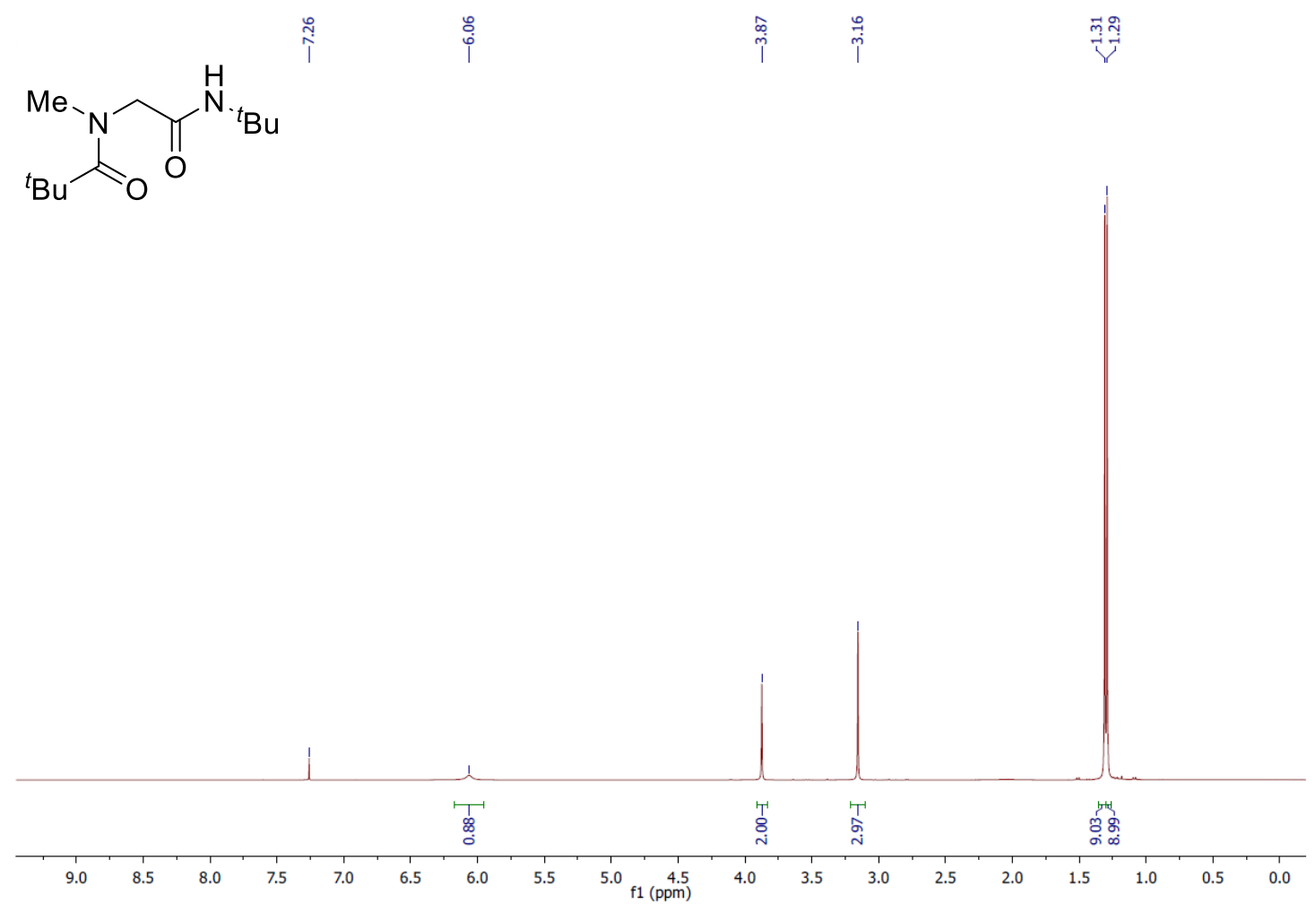

${ }^{13} \mathrm{C}\left\{{ }^{1} \mathrm{H}\right\} \mathrm{NMR}$ of 19 in $\mathrm{CDCl}_{3}$

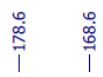

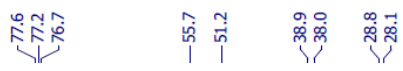<smiles>CN(CC(=O)NC(C)(C)C)C(=O)CC(C)(C)C</smiles>
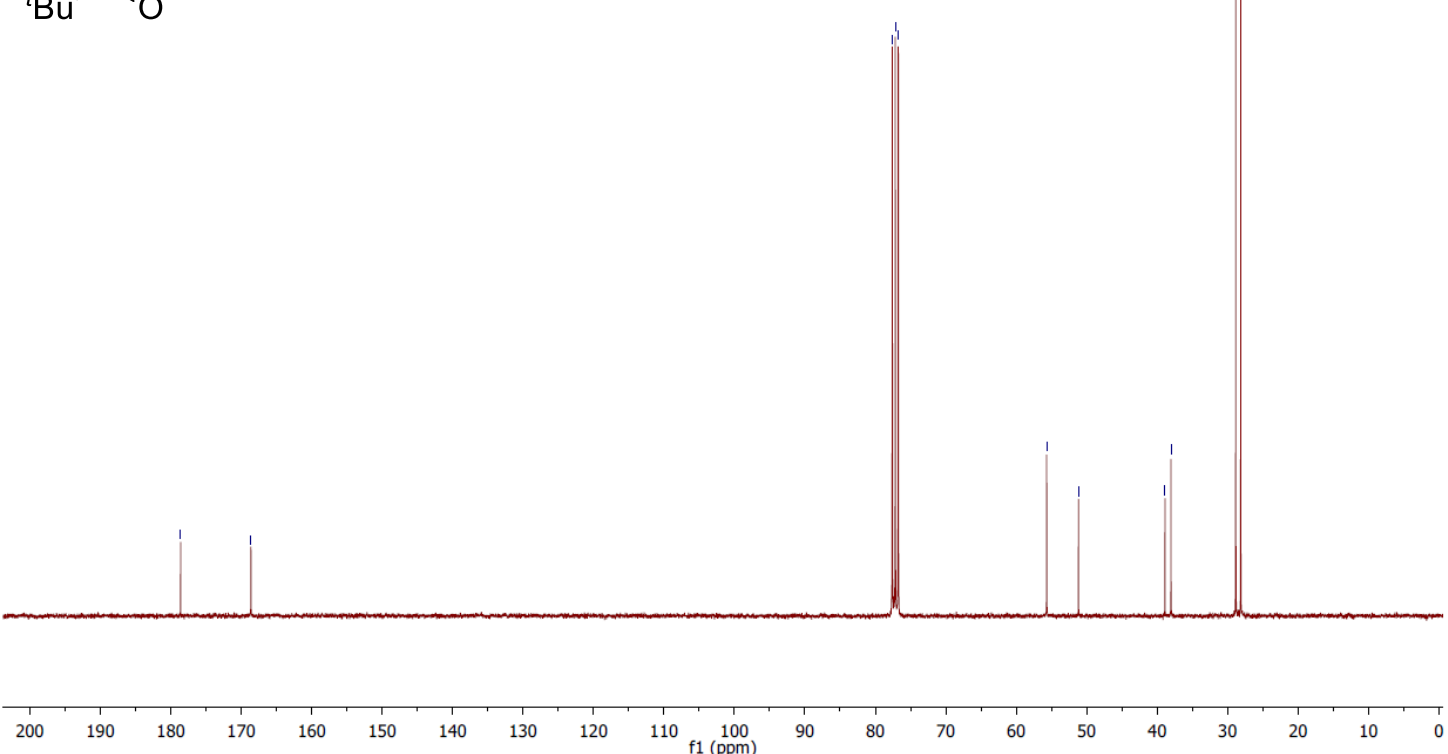
${ }^{1} \mathrm{H}$ NMR of 20 in $\mathrm{CDCl}_{3}$

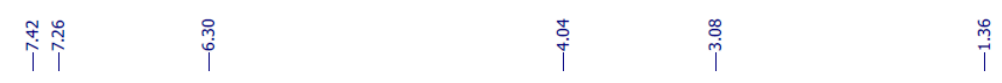<smiles>CN(CC(=O)NC(C)(C)C)C(=O)c1ccccc1</smiles>

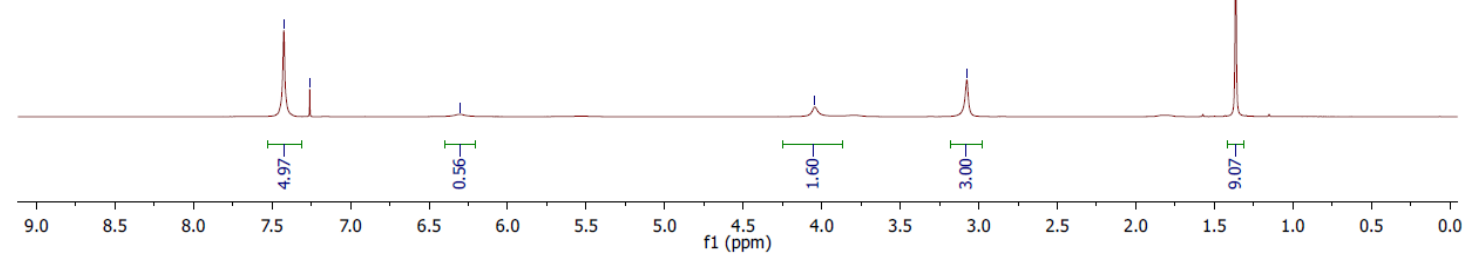

${ }^{13} \mathrm{C}\left\{{ }^{1} \mathrm{H}\right\}$ NMR of 20 in $\mathrm{CDCl}_{3}$

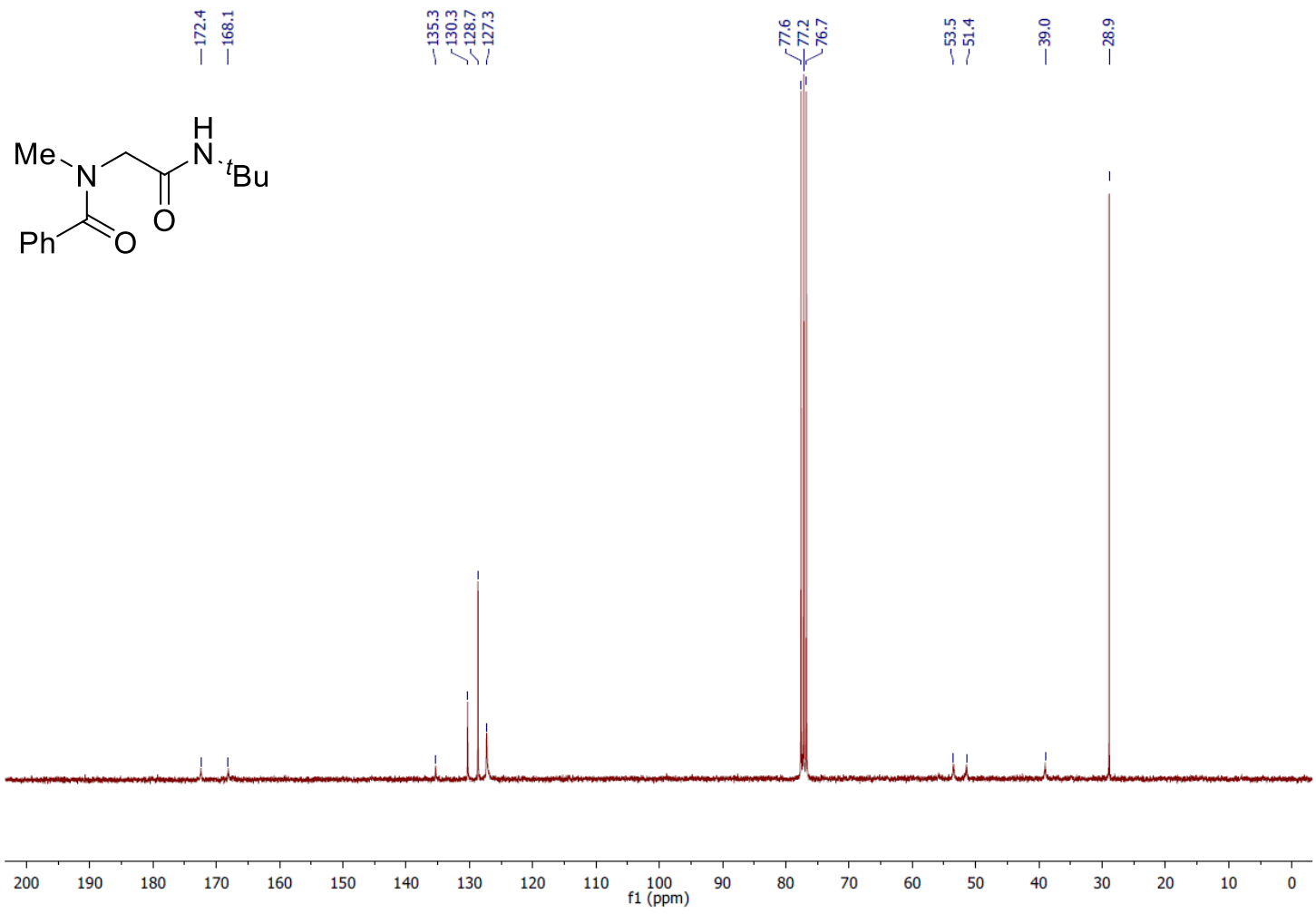


${ }^{1} \mathrm{H}$ NMR of 21 in $\mathrm{CDCl}_{3}$
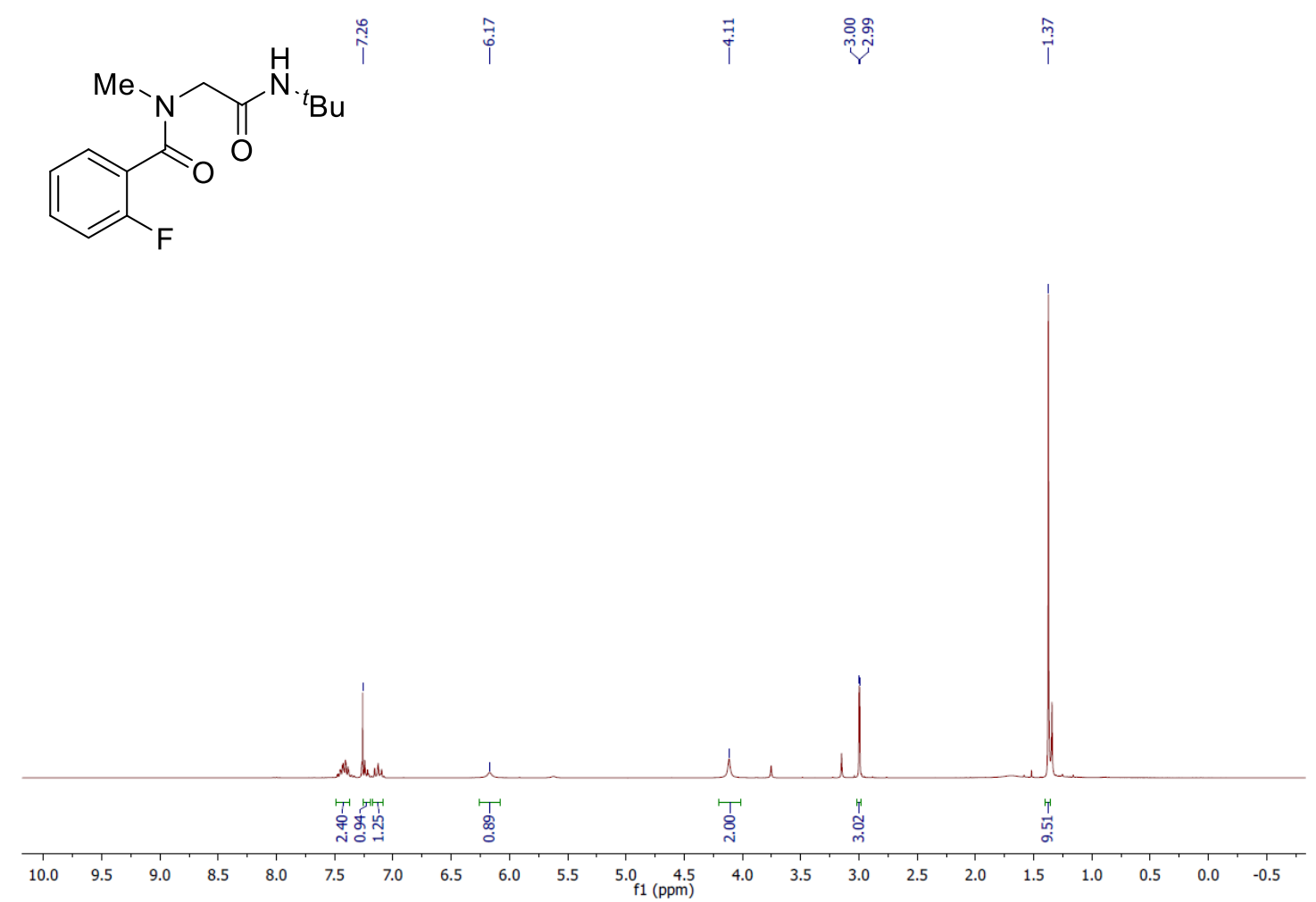

${ }^{13} \mathrm{C}\left\{{ }^{1} \mathrm{H}\right\}$ NMR of $\mathbf{2 1}$ in $\mathrm{CDCl}_{3}$

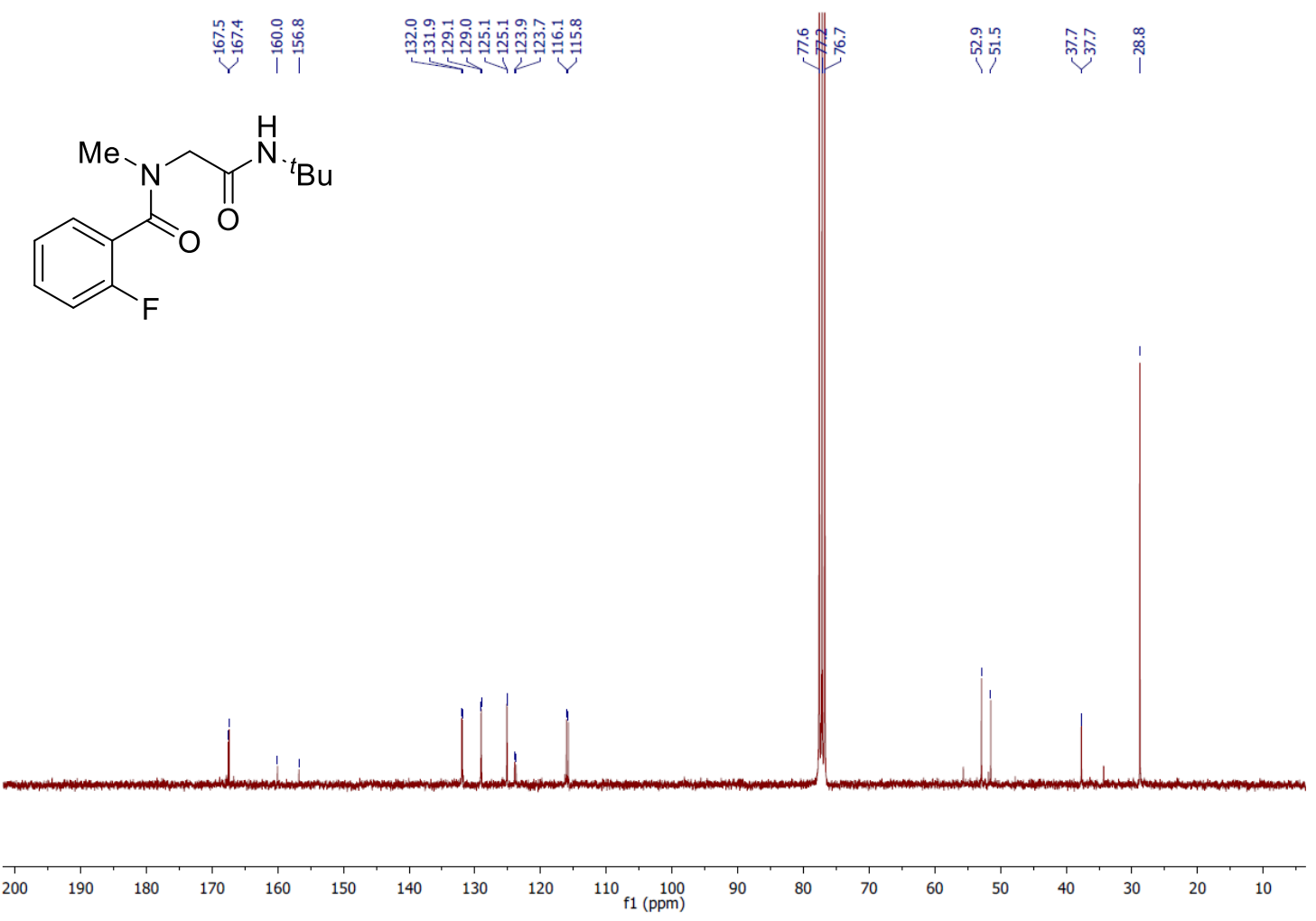


${ }^{19} \mathrm{~F}\left\{{ }^{1} \mathrm{H}\right\}$ NMR of 21 in $\mathrm{CDCl}_{3}$

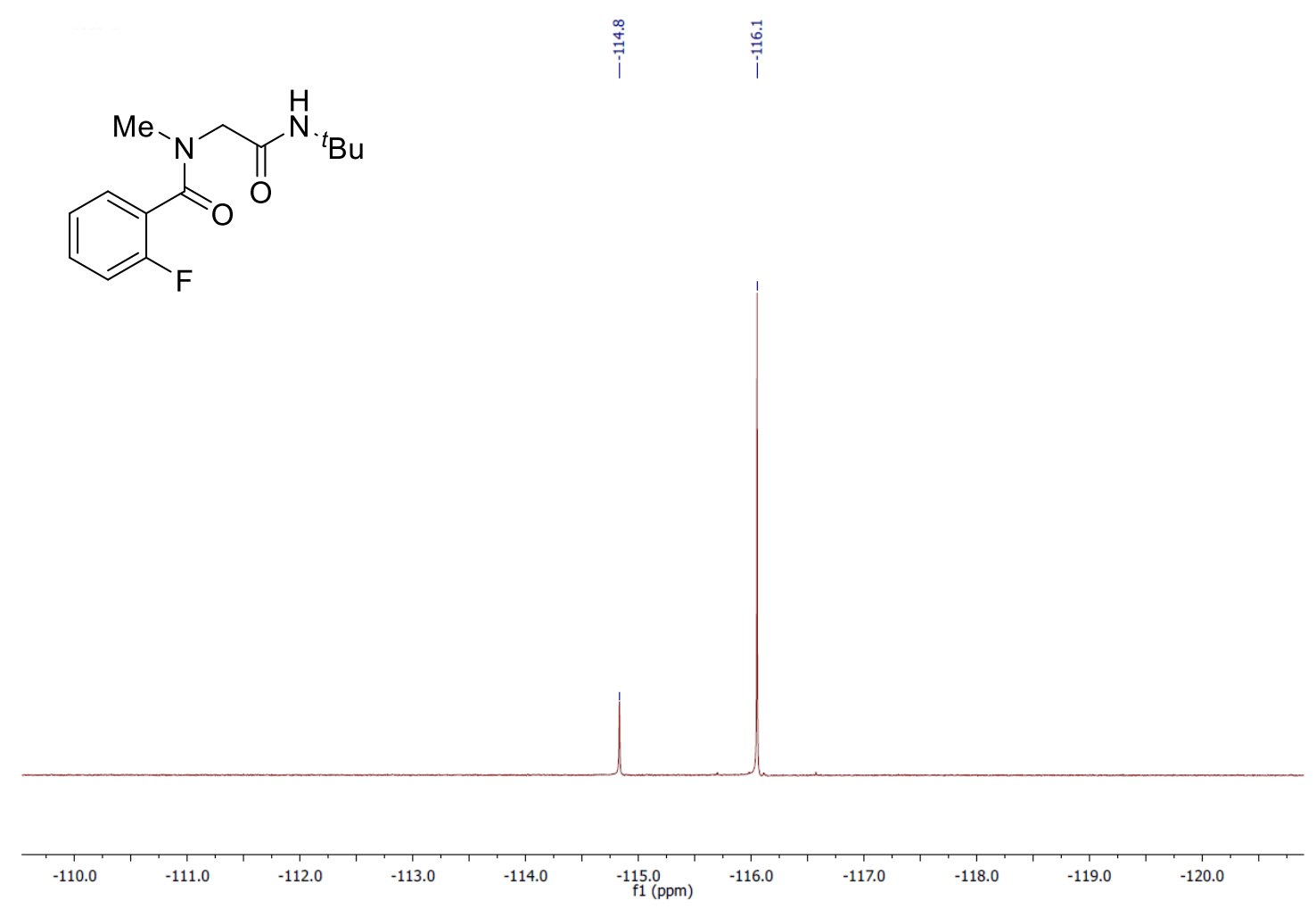

${ }^{1} \mathrm{H} \mathrm{NMR}$ of 22 in $\mathrm{CDCl}_{3}$

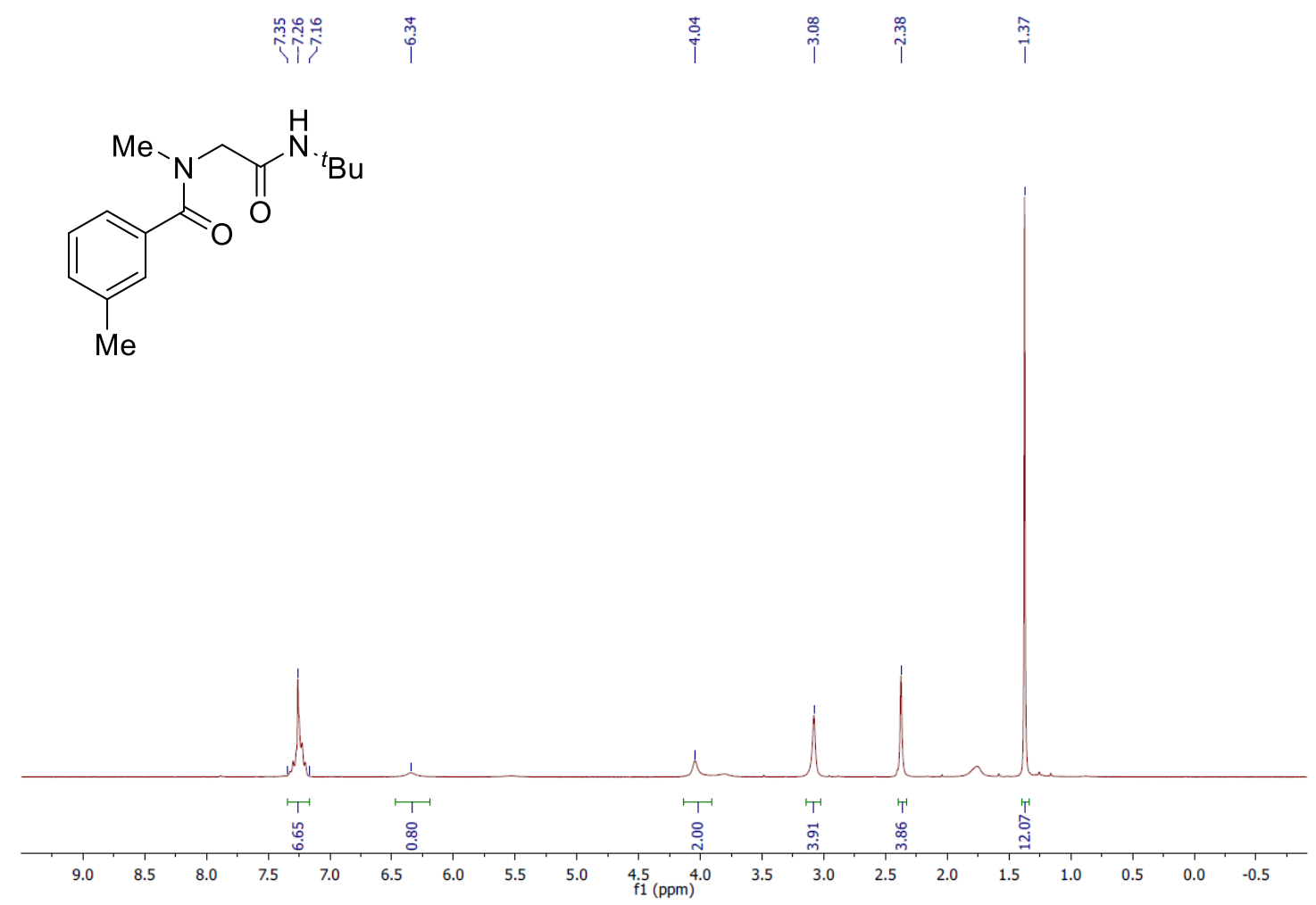


${ }^{13} \mathrm{C}\left\{{ }^{1} \mathrm{H}\right\} \mathrm{NMR}$ of 22 in $\mathrm{CDCl}_{3}$

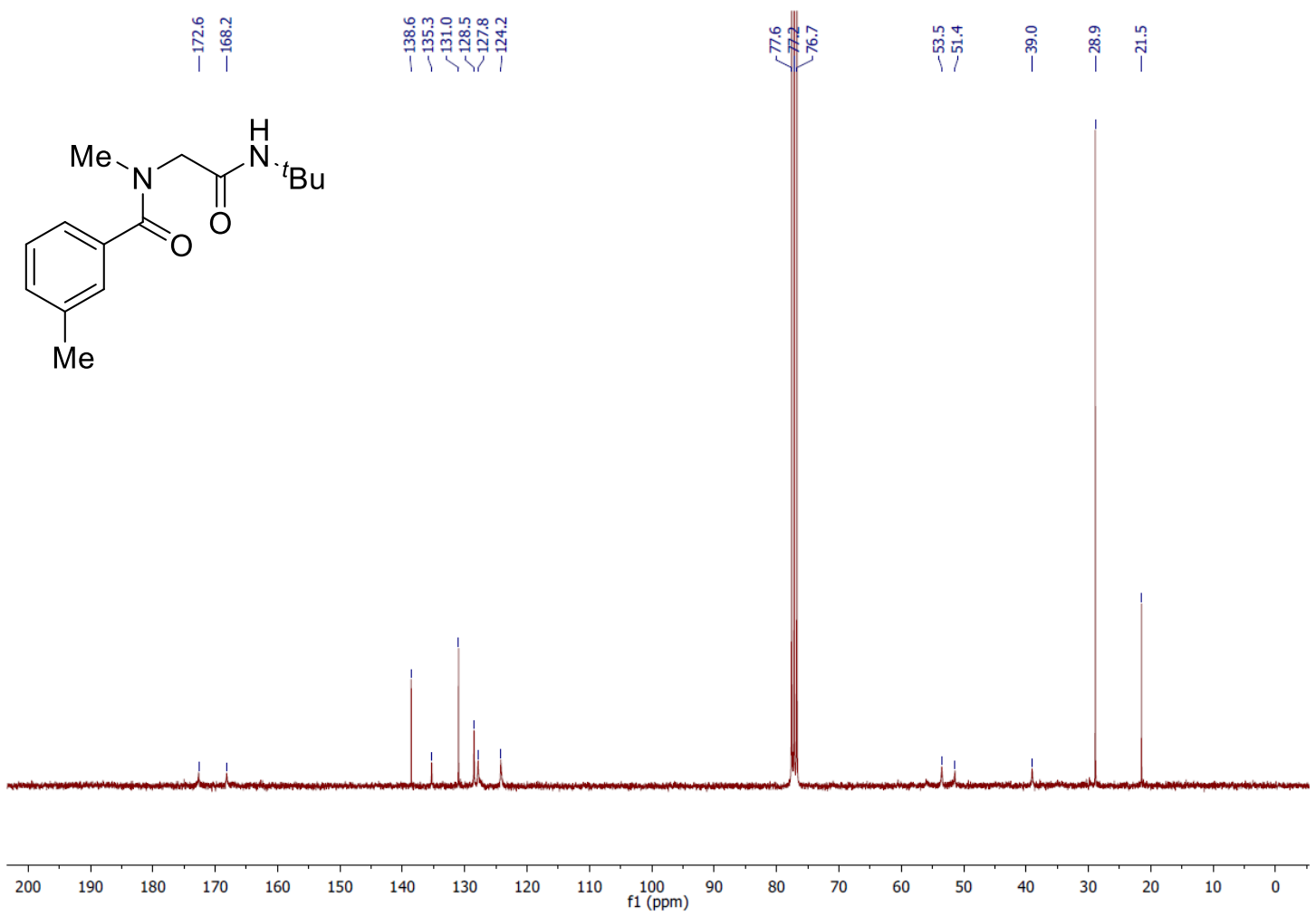

${ }^{1} \mathrm{H}$ NMR of 23 in $\mathrm{CDCl}_{3}$

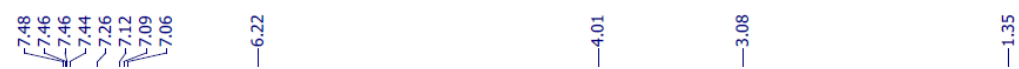<smiles>CCC(C)NC(=O)CN(C)C(=O)c1ccc(F)cc1</smiles>

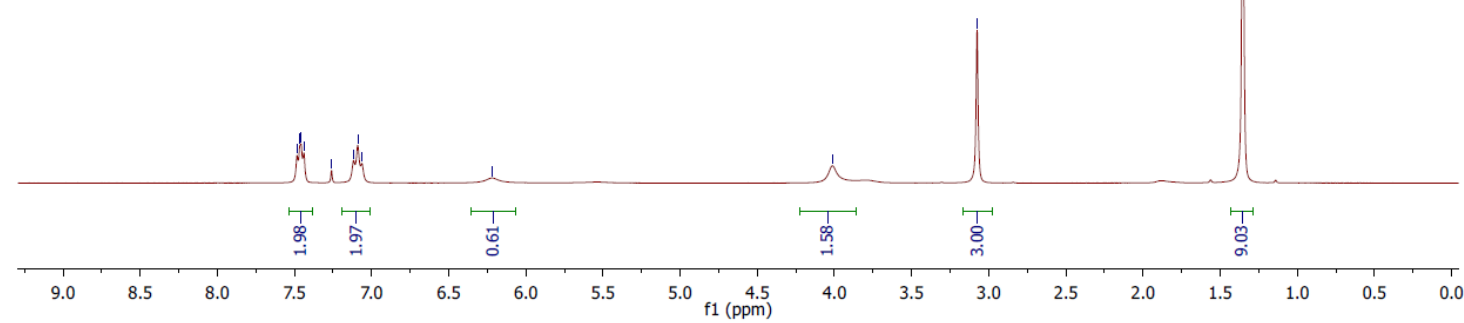


${ }^{13} \mathrm{C}\left\{{ }^{1} \mathrm{H}\right\}$ NMR of 23 in $\mathrm{CDCl}_{3}$

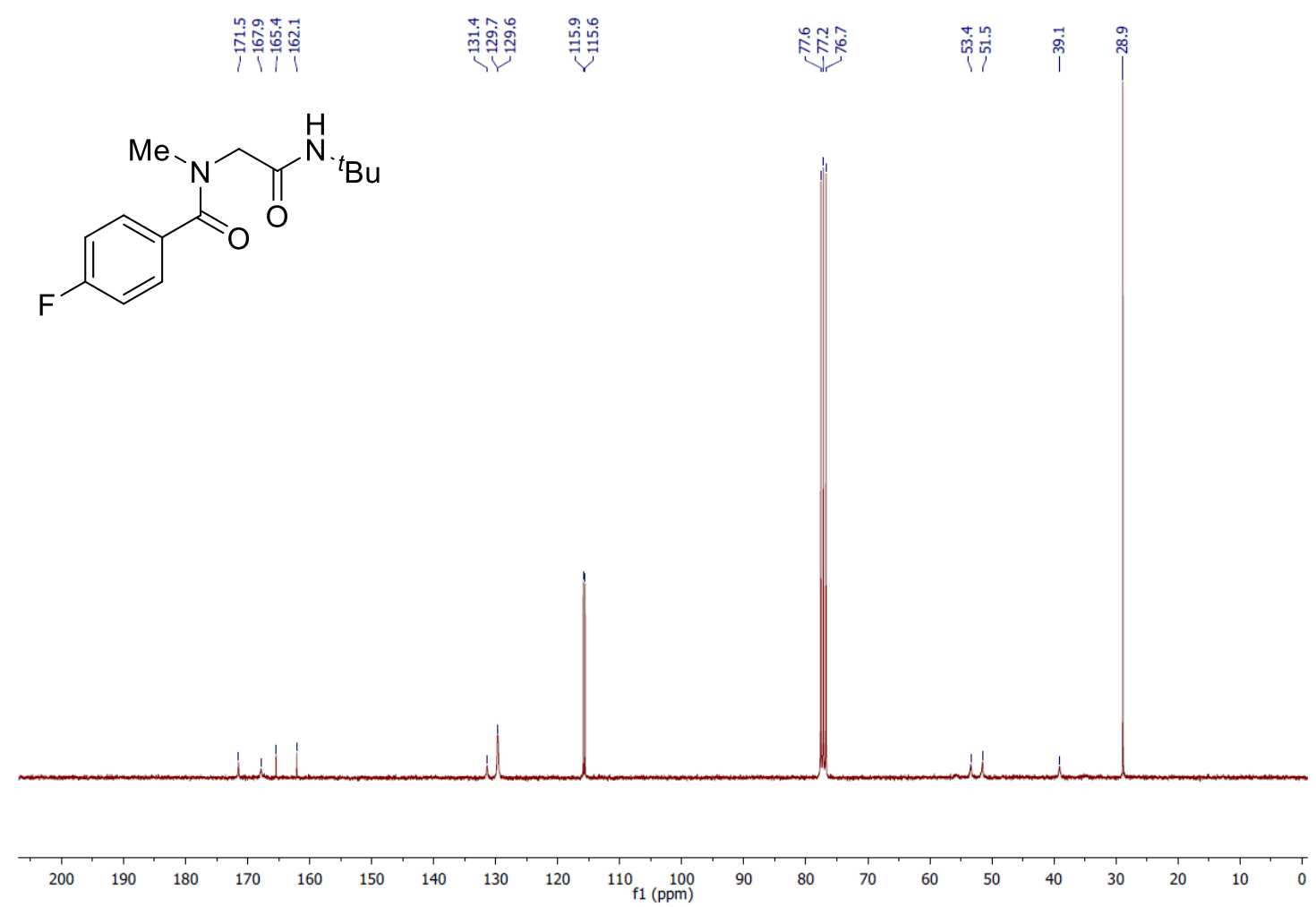

${ }^{19} \mathrm{~F}\left\{{ }^{1} \mathrm{H}\right\} \mathrm{NMR}$ of 23 in $\mathrm{CDCl}_{3}$<smiles>CCC(C)NC(=O)CN(C)C(=O)c1ccc(F)cc1</smiles>

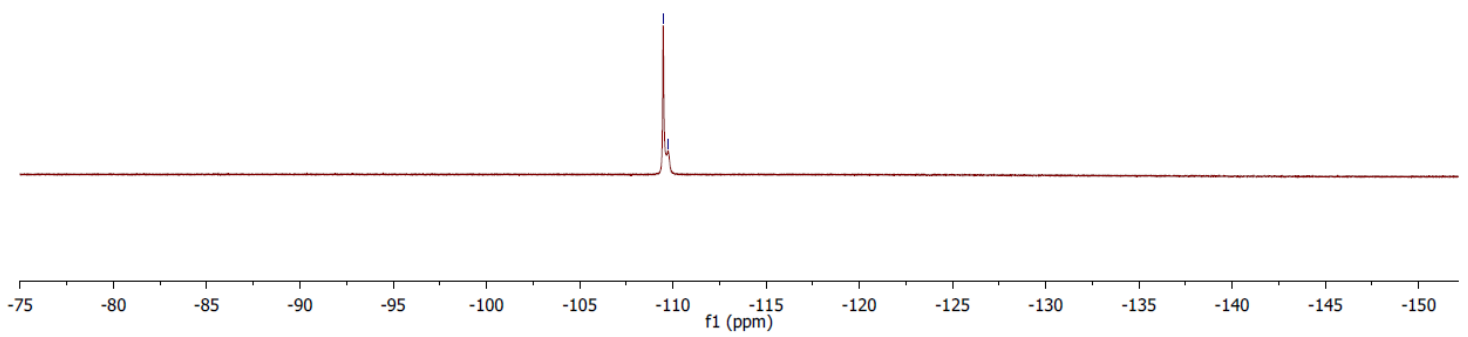


${ }^{1} \mathrm{H}$ NMR of 24 in $\mathrm{CDCl}_{3}$

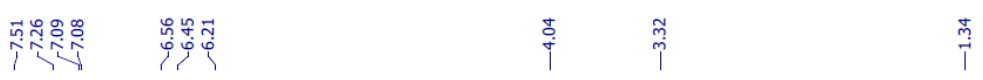<smiles>CCCCC(=O)N(C)CC(=O)c1ccco1</smiles>

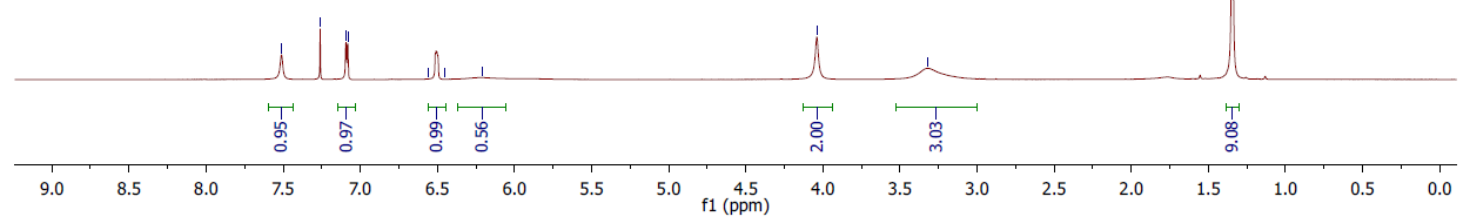

${ }^{13} \mathrm{C}\left\{{ }^{1} \mathrm{H}\right\} \mathrm{NMR}$ of 24 in $\mathrm{CDCl}_{3}$

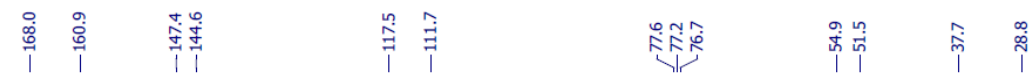<smiles>CN(CC(=O)NC(C)(C)C)C(=O)c1ccco1</smiles> 
${ }^{1} \mathrm{H}$ NMR of 25 in $\mathrm{CDCl}_{3}$

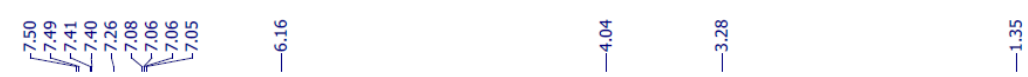<smiles>CN(CC(=O)NC(C)(C)C)C(=O)c1cccs1</smiles>

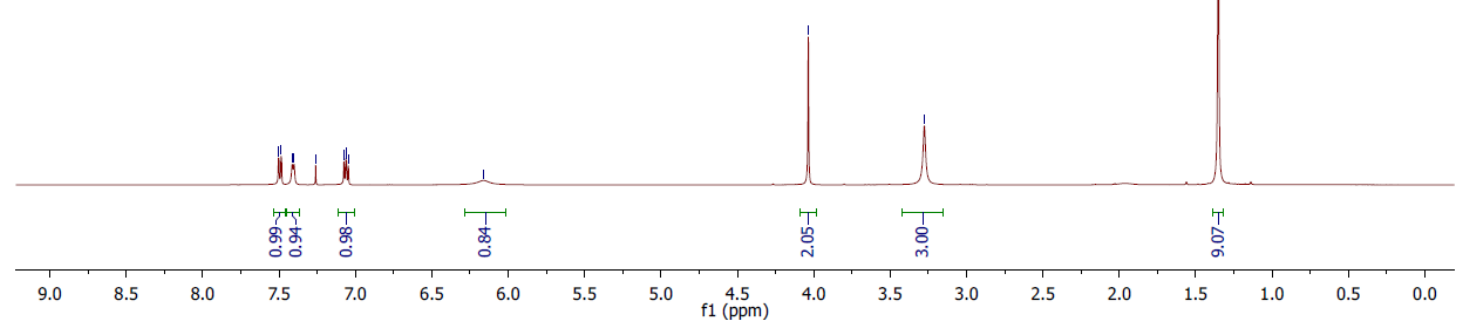

${ }^{13} \mathrm{C}\left\{{ }^{1} \mathrm{H}\right\} \mathrm{NMR}$ of 25 in $\mathrm{CDCl}_{3}$

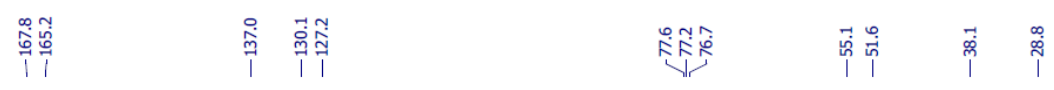<smiles>CCC(C)NC(=O)CN(C)C(=O)c1cccs1</smiles>
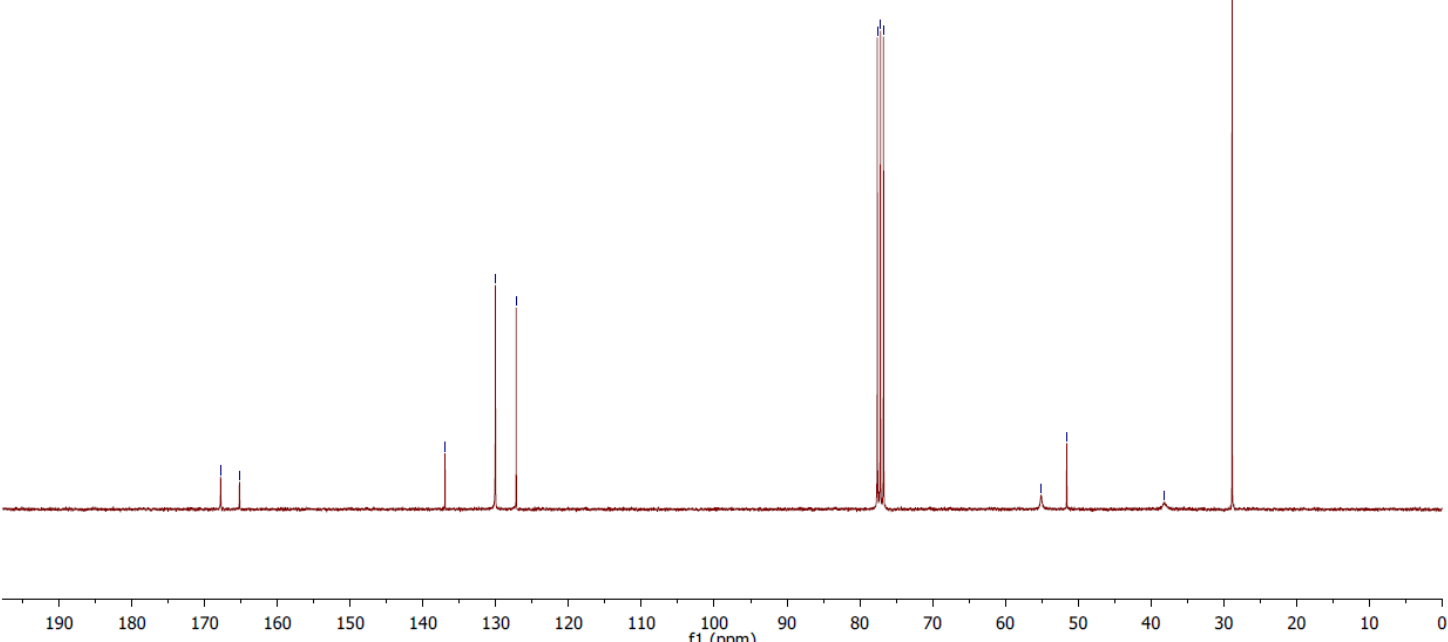
$\mathrm{HSQC}$ of $\mathbf{2 5}$ in $\mathrm{CDCl}_{3}$

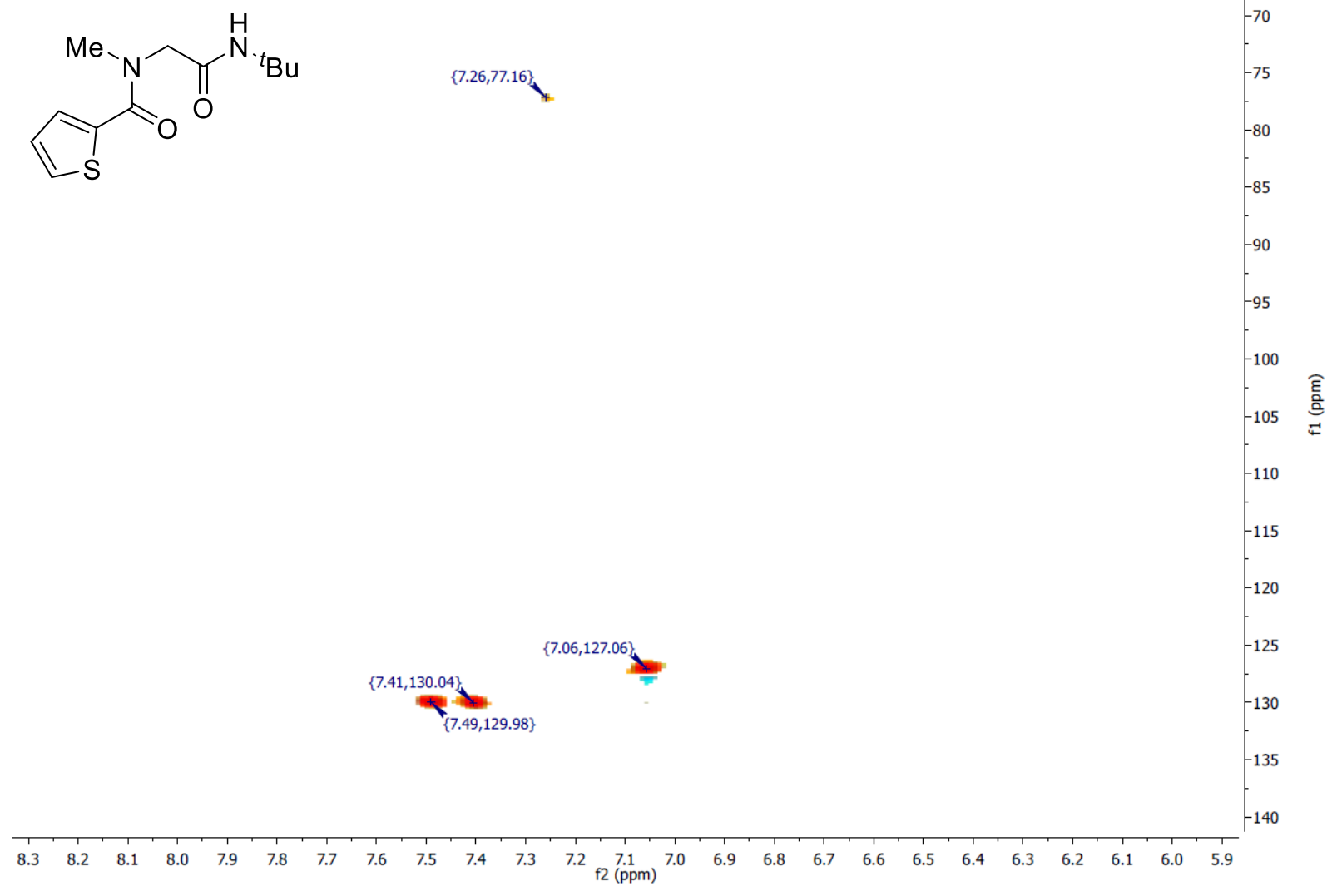


${ }^{1} \mathrm{H} \mathrm{NMR}$ of 26 in $\mathrm{CDCl}_{3}$

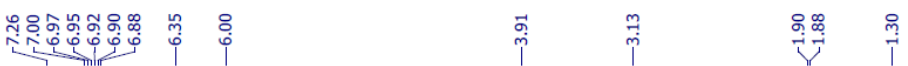<smiles>C/C=C/C(=O)N(C)CC(=O)NC(C)(C)C</smiles>

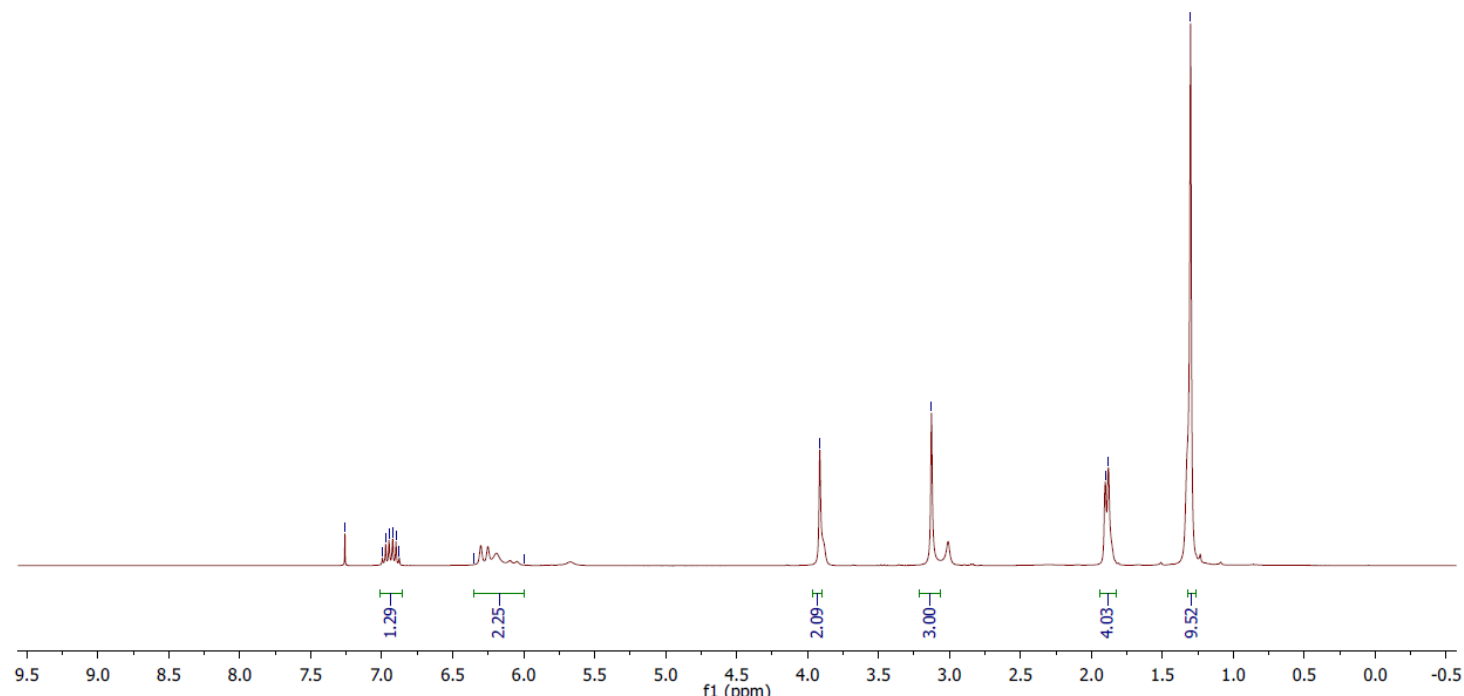

${ }^{13} \mathrm{C}\left\{{ }^{1} \mathrm{H}\right\} \mathrm{NMR}$ of $\mathbf{2 6}$ in $\mathrm{CDCl}_{3}$

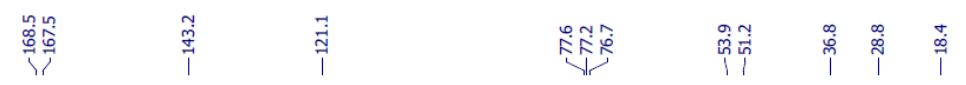<smiles>C/C=C/C(=O)N(C)CC(=O)NC(C)(C)C</smiles>

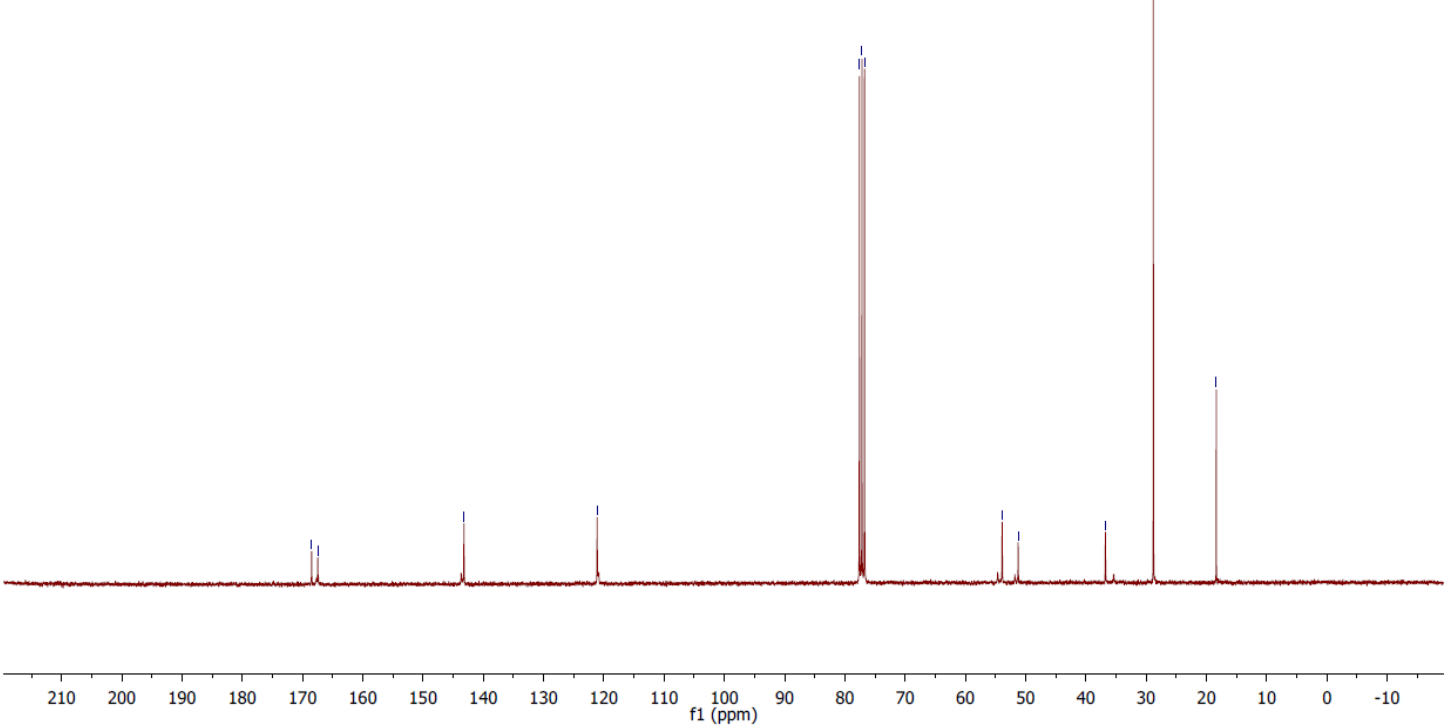


${ }^{1} \mathrm{H}$ NMR of 27 in $\mathrm{CDCl}_{3}$

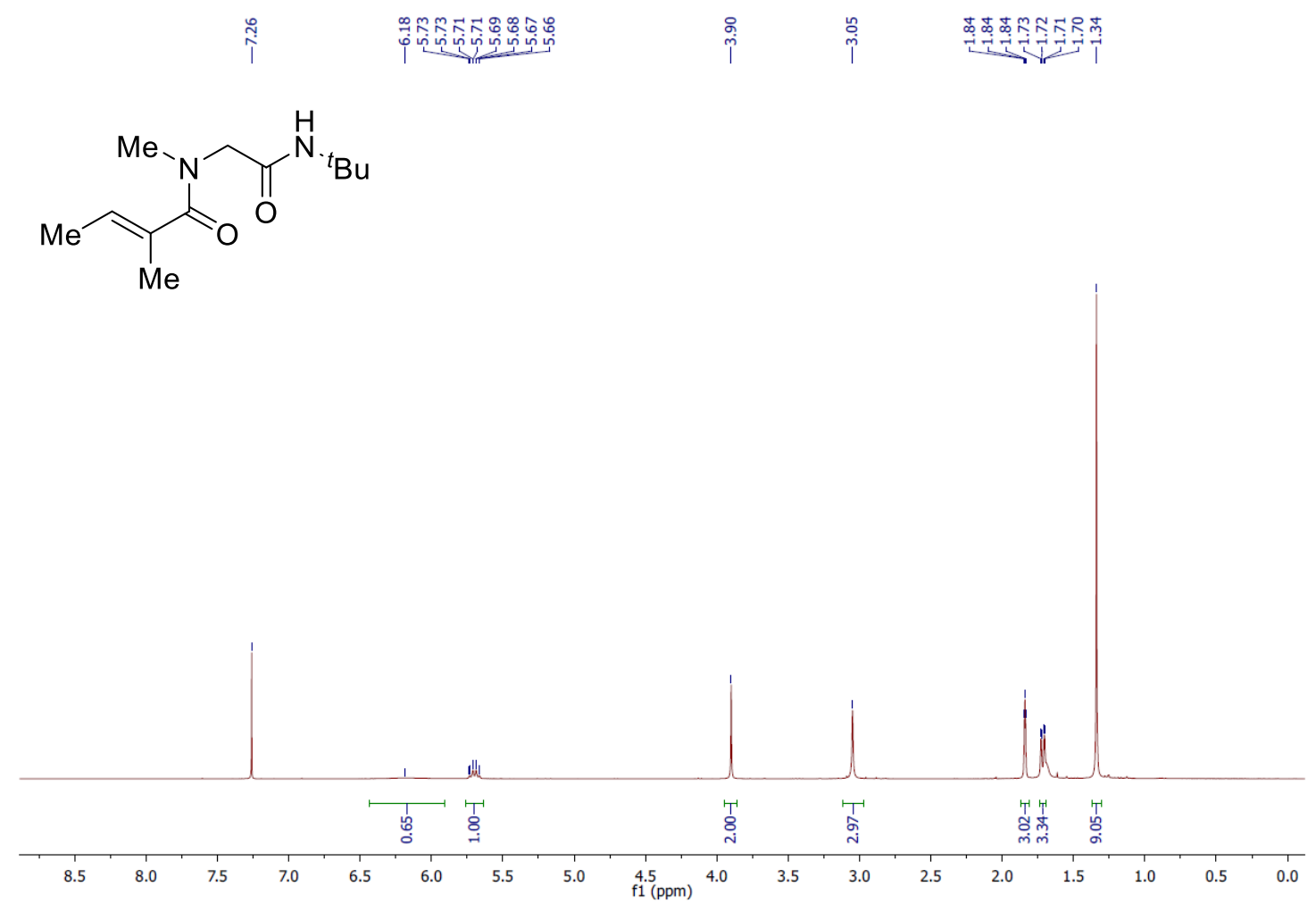

${ }^{13} \mathrm{C}\left\{{ }^{1} \mathrm{H}\right\} \mathrm{NMR}$ of 27 in $\mathrm{CDCl}_{3}$

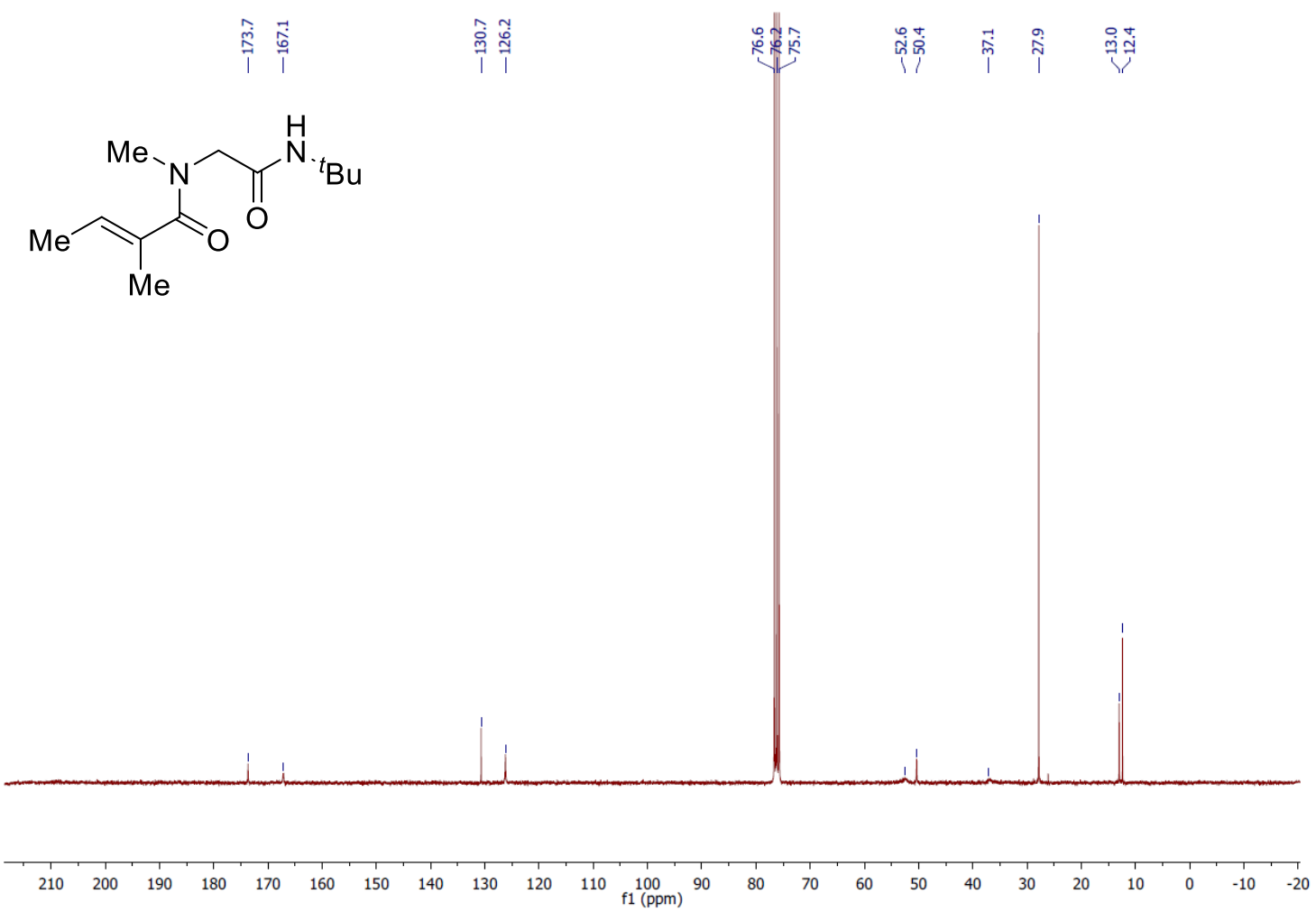


${ }^{1} \mathrm{H}$ NMR of 28 in $\mathrm{CDCl}_{3}$
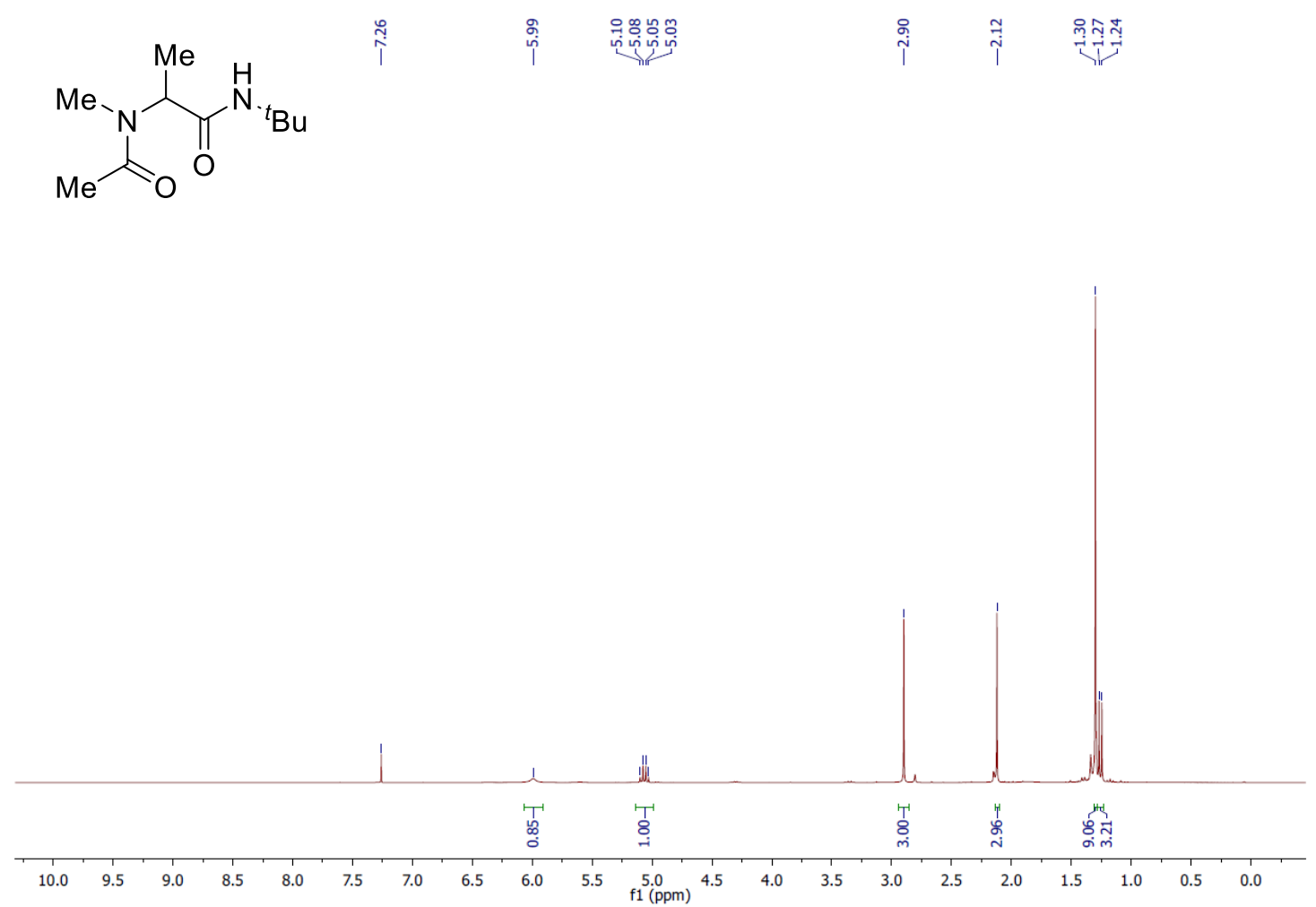

${ }^{13} \mathrm{C}\left\{{ }^{1} \mathrm{H}\right\}$ NMR of $\mathbf{2 8}$ in $\mathrm{CDCl}_{3}$

i.

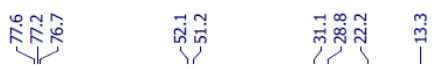<smiles>CC(=O)N(C)C(C)C(=O)NC(C)(C)C</smiles>

$\begin{array}{lllllllllllllllllllllllllllll}210 & 200 & 190 & 180 & 170 & 160 & 150 & 140 & 130 & 120 & 110 & 100 & 90 & 80 & 70 & 60 & 50 & 40 & 30 & 20 & 10 & 0 & -10\end{array}$ 
${ }^{1} \mathrm{H}$ NMR of 29 in $\mathrm{CDCl}_{3}$

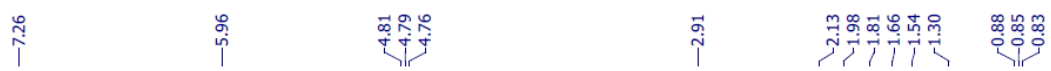<smiles>CCC(C(=O)NC(C)(C)C)N(C)C(C)=O</smiles>

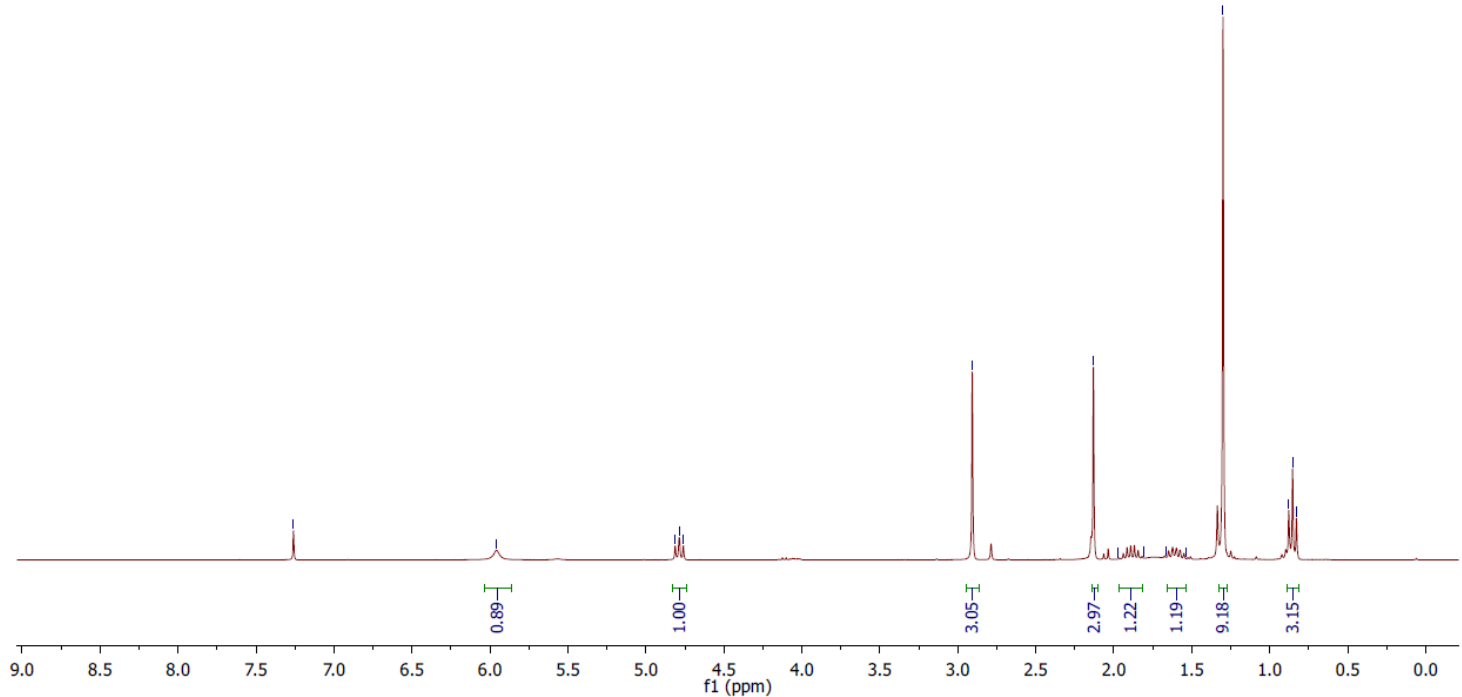

${ }^{13} \mathrm{C}\left\{{ }^{1} \mathrm{H}\right\} \mathrm{NMR}$ of 29 in $\mathrm{CDCl}_{3}$

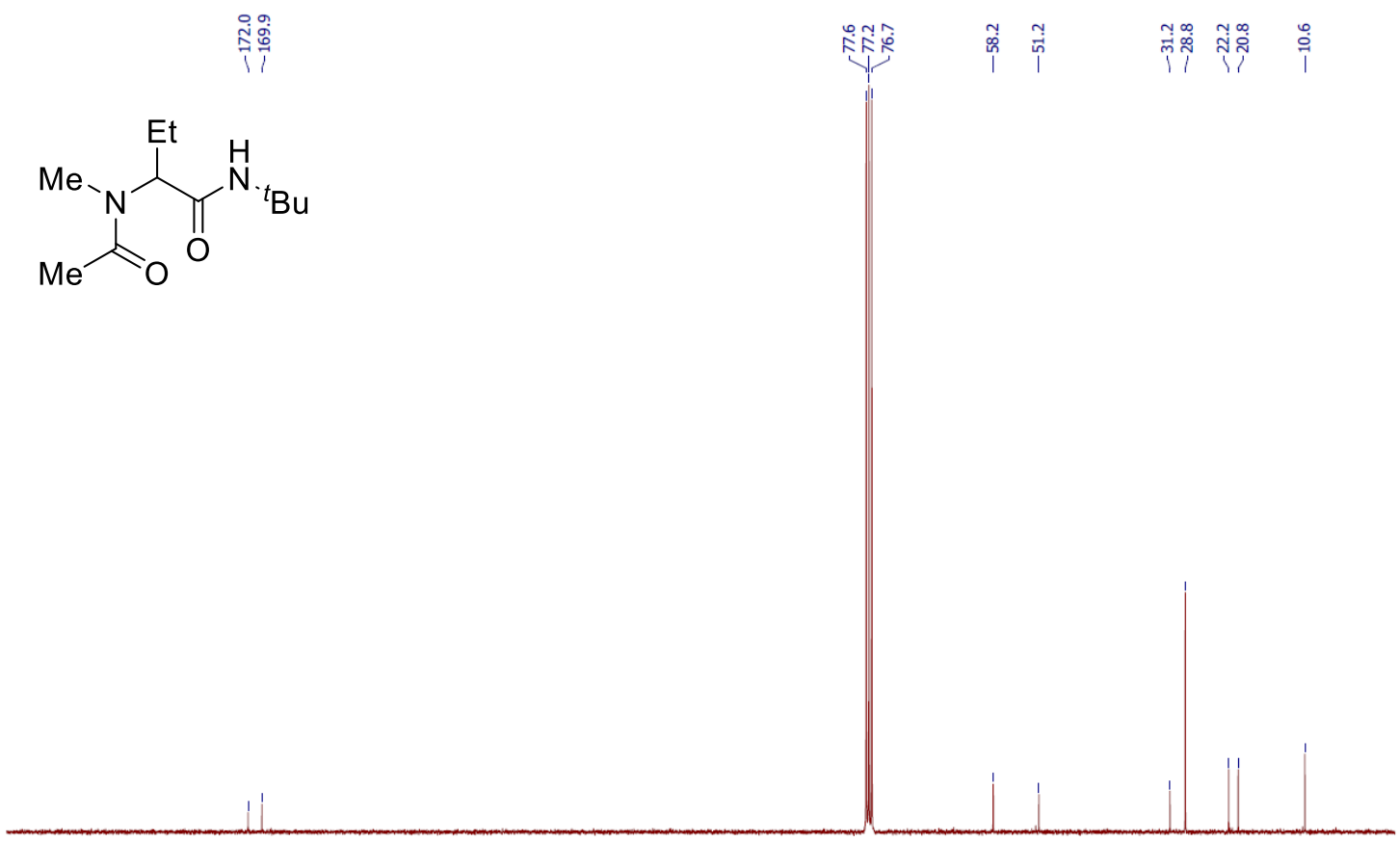

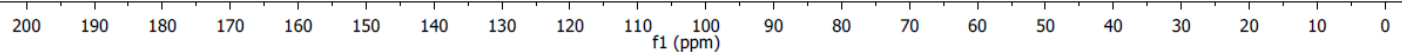


${ }^{1} \mathrm{H}$ NMR of 30 in $\mathrm{CDCl}_{3}$

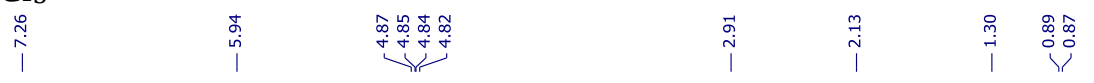<smiles>CCC(CCCCCCCCC(C)(C)C)N(C)C(C)=O</smiles>

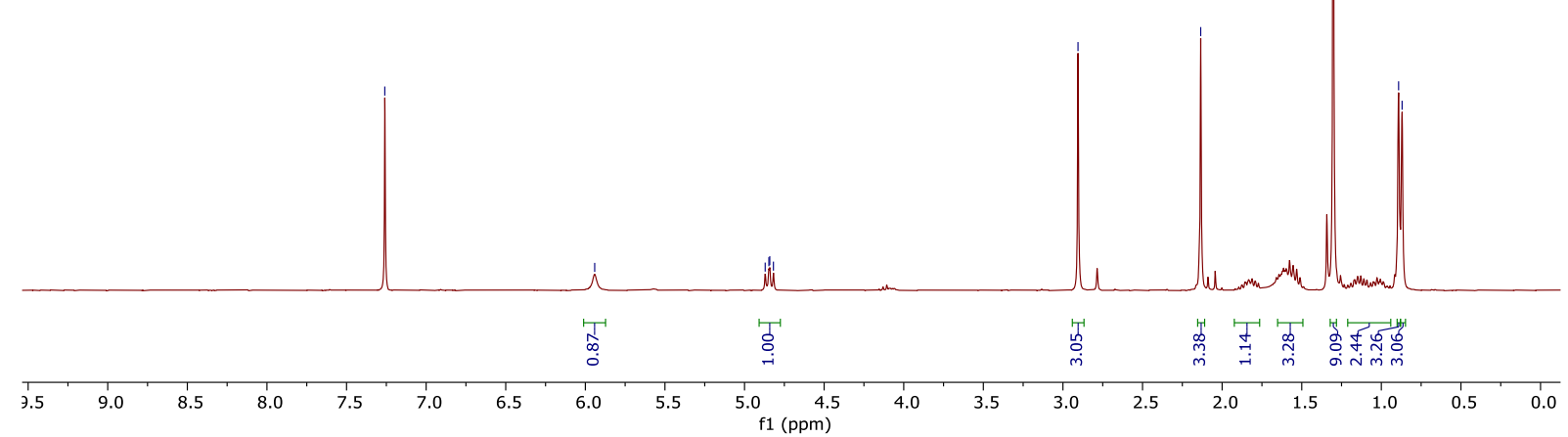
${ }^{13} \mathrm{C}\left\{{ }^{1} \mathrm{H}\right\} \mathrm{NMR}$ of $\mathbf{3 0}$ in $\mathrm{CDCl}_{3}$
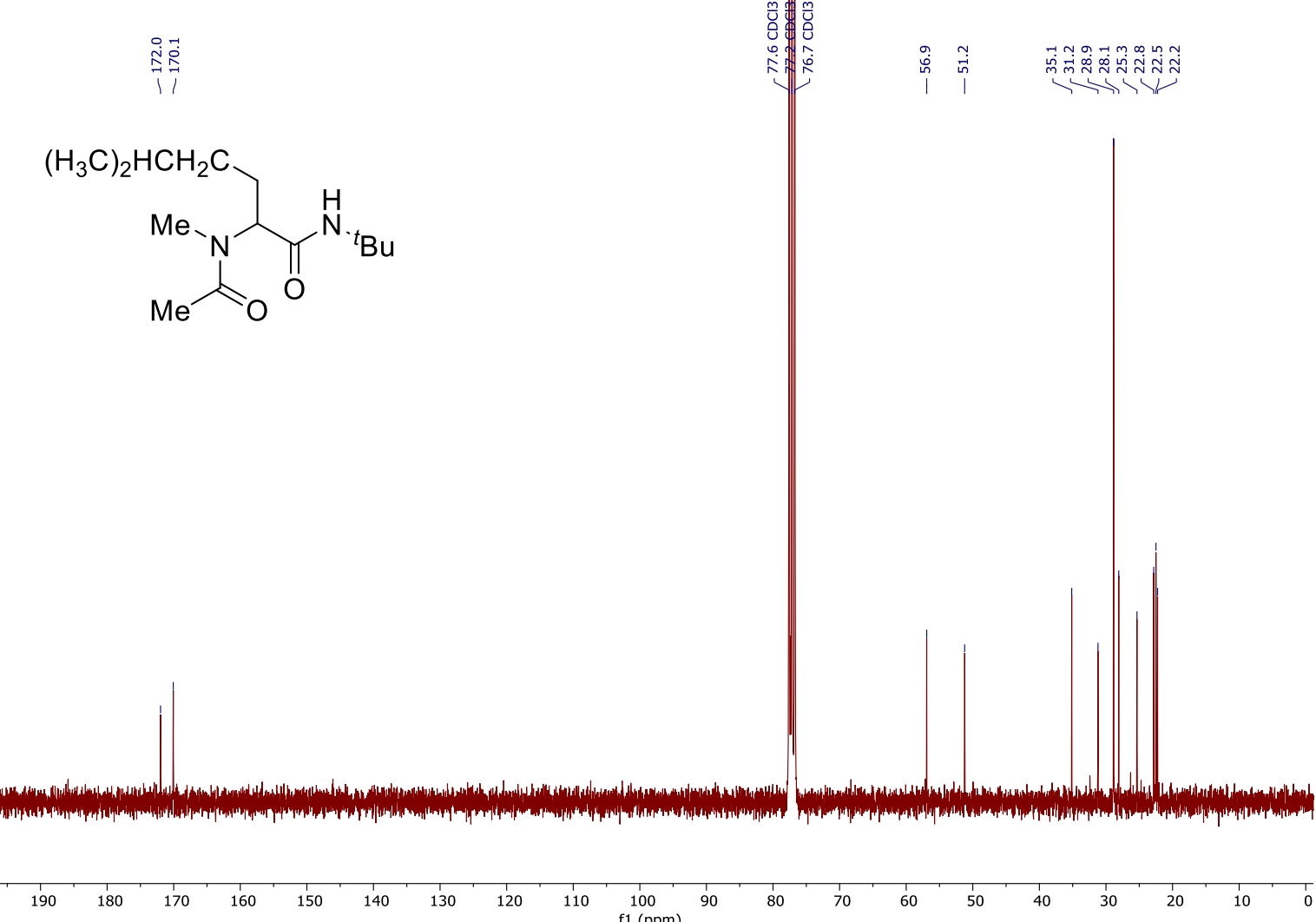
${ }^{1} \mathrm{H}$ NMR of 31 in $\mathrm{CDCl}_{3}$

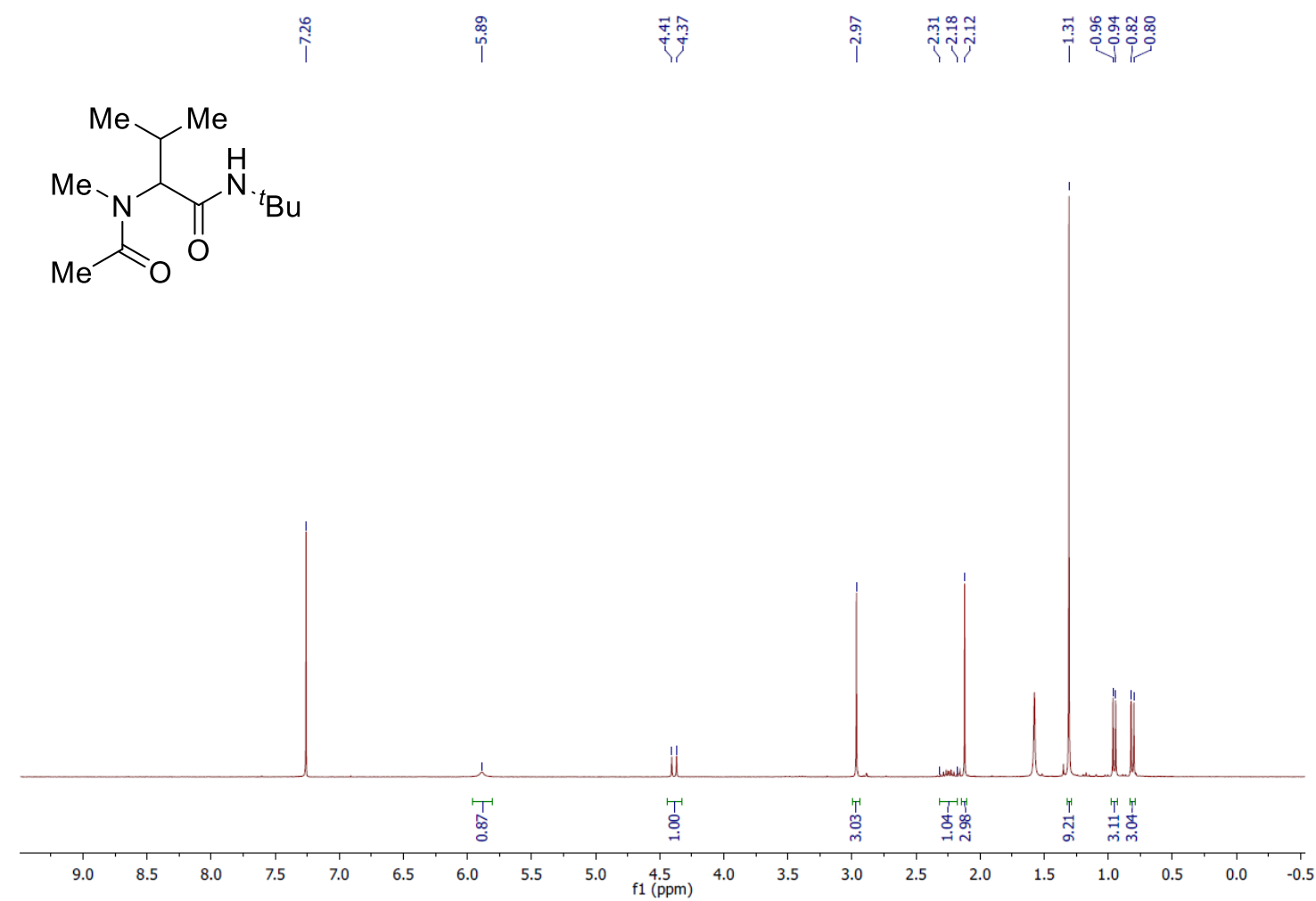

${ }^{13} \mathrm{C}\left\{{ }^{1} \mathrm{H}\right\}$ NMR of $\mathbf{3 1}$ in $\mathrm{CDCl}_{3}$

昰照

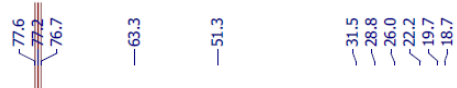

$\overbrace{O}^{M e} \underbrace{M e}_{O}$

$\begin{array}{llllllllll}190 & 180 & 170 & 160 & 150 & 140 & 130 & 120 & 110 & 100 \\ \mathrm{f} 1(\mathrm{ppm}) & 90\end{array}$ 
${ }^{1} \mathrm{H}$ NMR of 32 in $\mathrm{CDCl}_{3}$
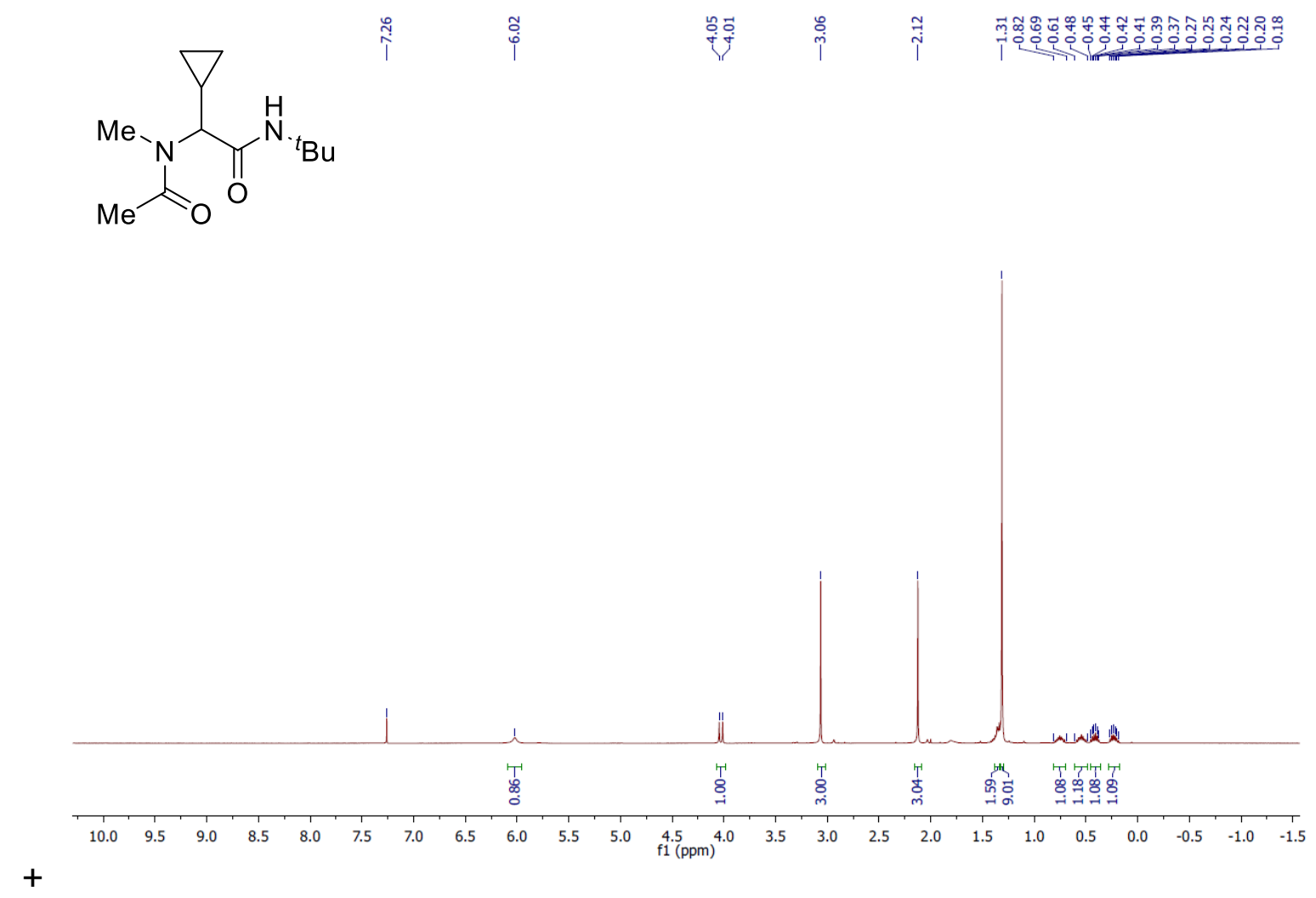

${ }^{13} \mathrm{C}\left\{{ }^{1} \mathrm{H}\right\} \mathrm{NMR}$ of 32 in $\mathrm{CDCl}_{3}$

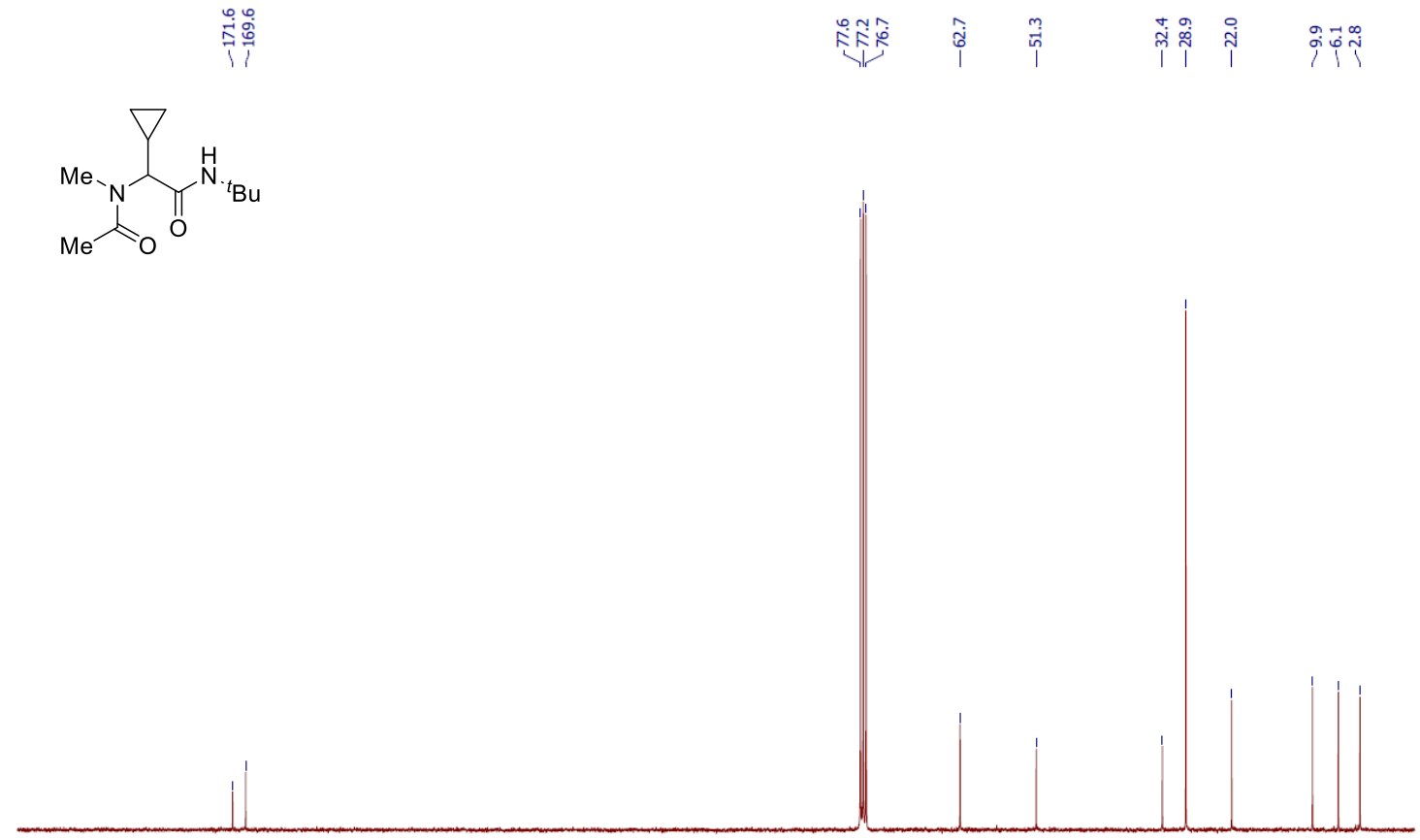

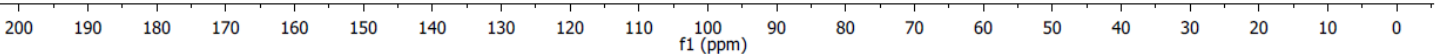


${ }^{1} \mathrm{H}$ NMR of 33 in $\mathrm{CDCl}_{3}$

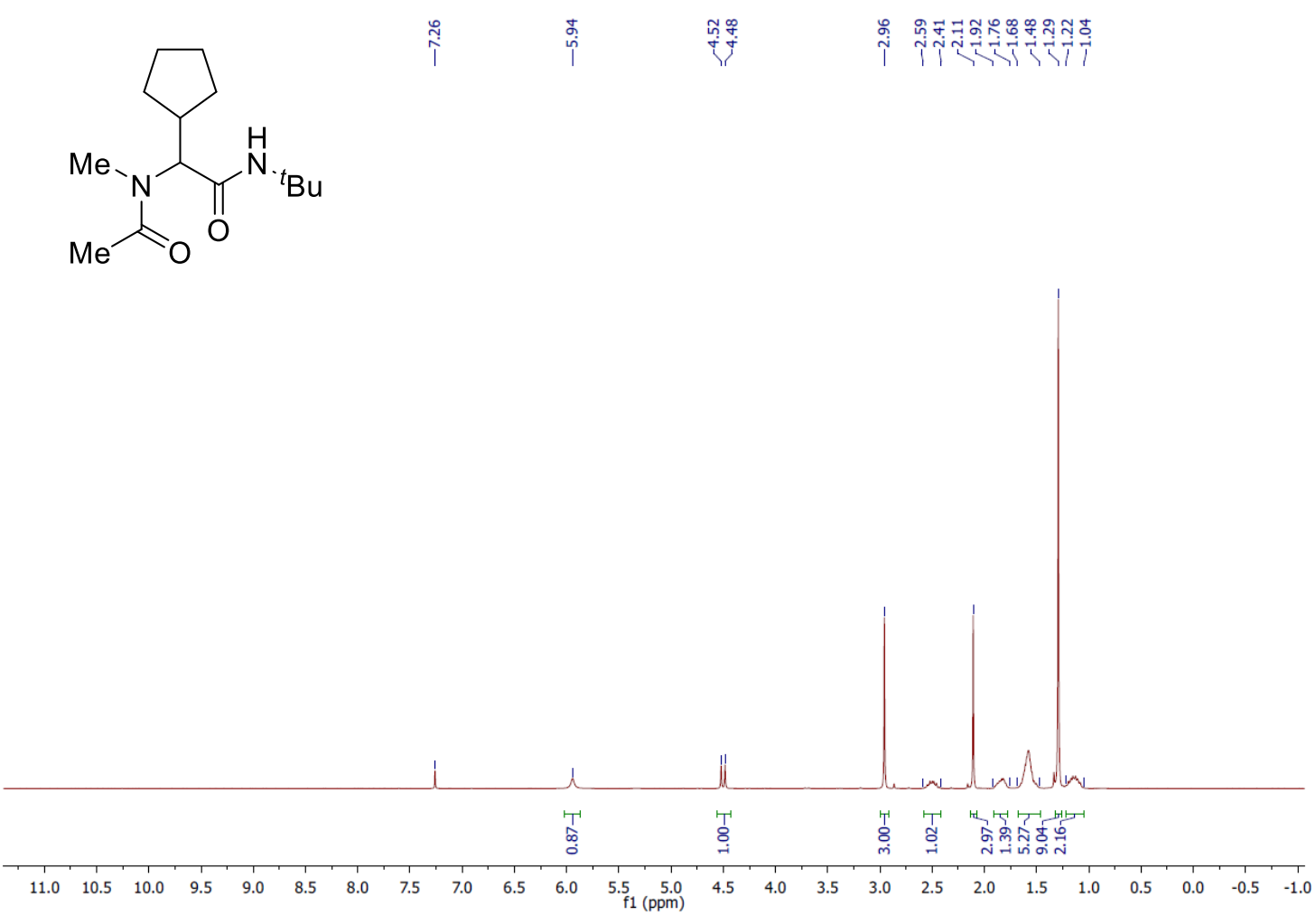

${ }^{13} \mathrm{C}\left\{{ }^{1} \mathrm{H}\right\} \mathrm{NMR}$ of 33 in $\mathrm{CDCl}_{3}$

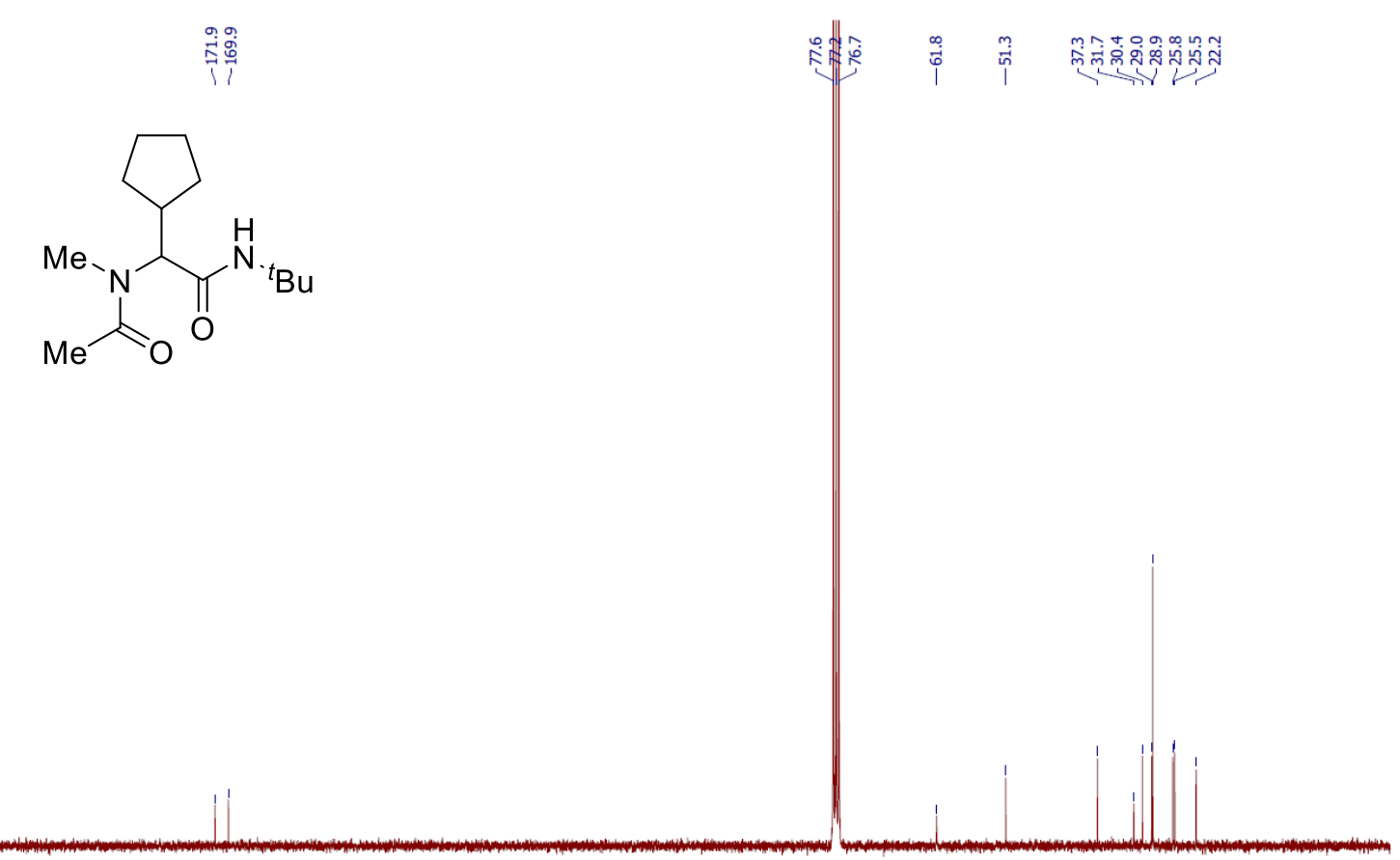

$\begin{array}{lllllllllllllllllllllllllll} & 1 & 1 & 1 & 1 & 180 & 170 & 160 & 150 & 140 & 130 & 120 & 110 & 100 & 90 & 80 & 70 & 60 & 50 & 40 & 30 & 20 & 10 & 0\end{array}$ 
${ }^{1} \mathrm{H}$ NMR of 34 in $\mathrm{CDCl}_{3}$
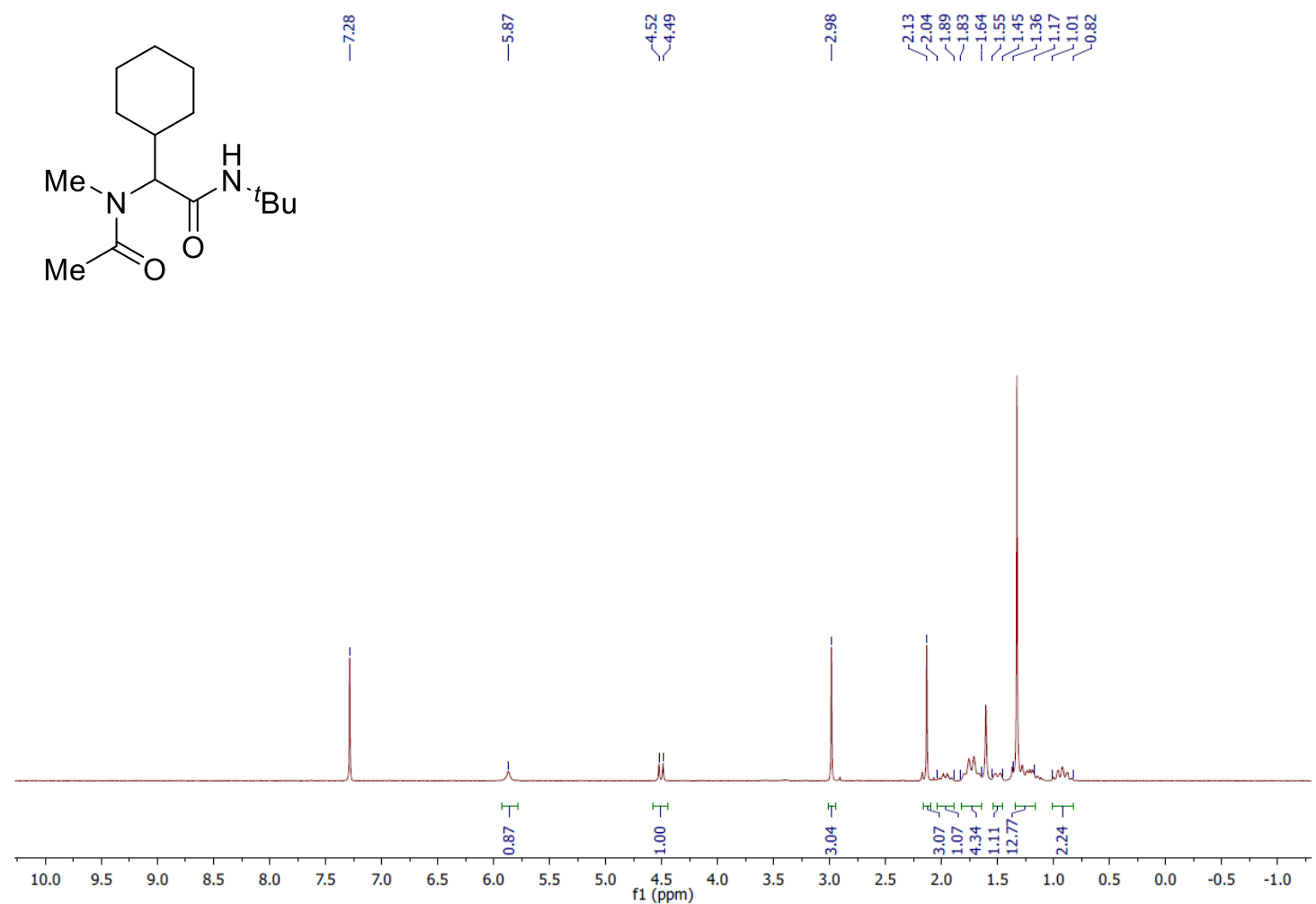

${ }^{13} \mathrm{C}\left\{{ }^{1} \mathrm{H}\right\}$ NMR of 34 in $\mathrm{CDCl}_{3}$

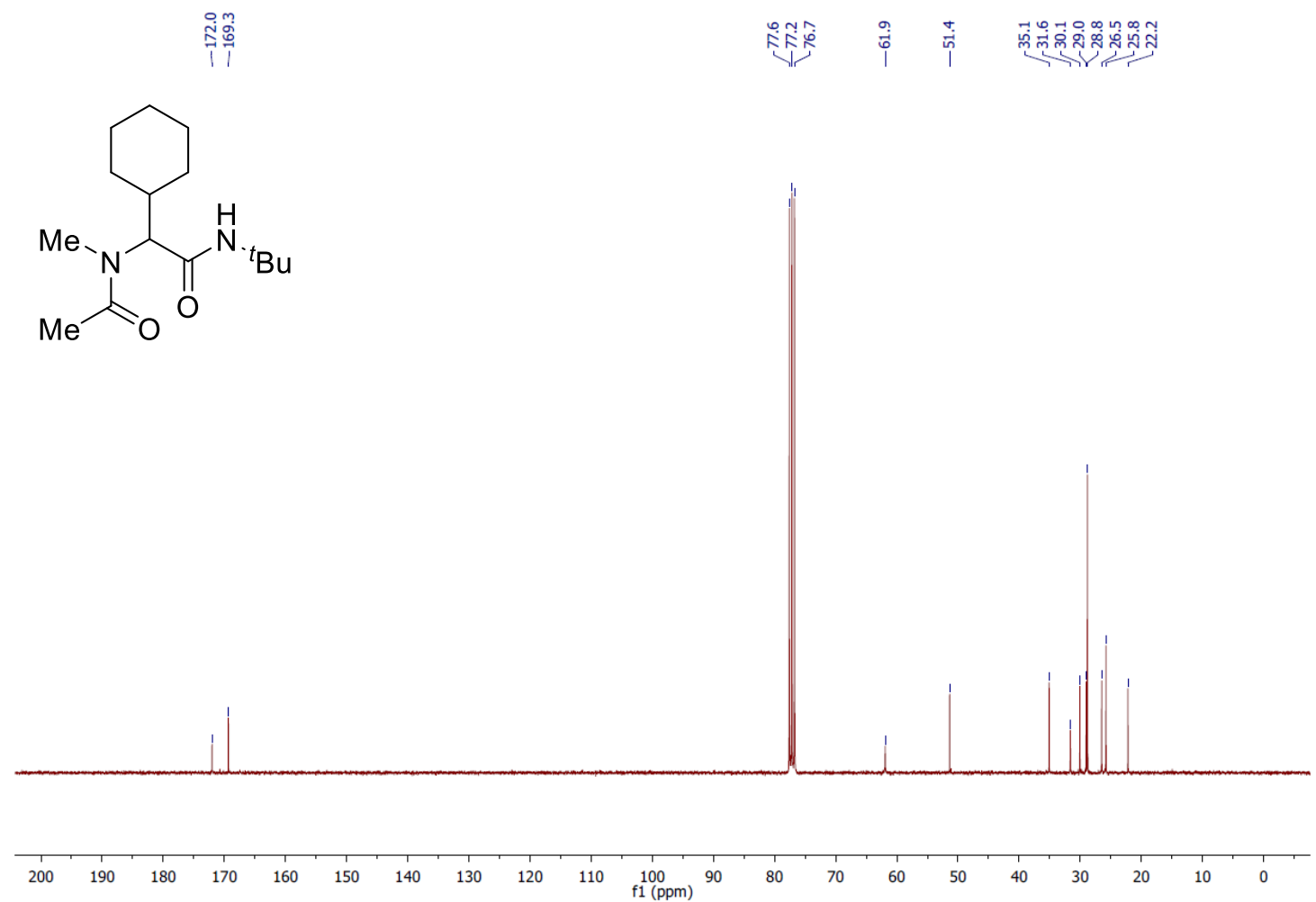


${ }^{1} \mathrm{H}$ NMR of 35 in $\mathrm{CDCl}_{3}$<smiles>CC(=O)N(C)C(Cc1ccccc1)C(=O)NC(C)(C)C</smiles>

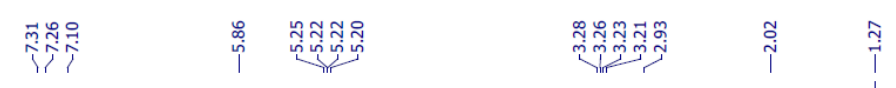

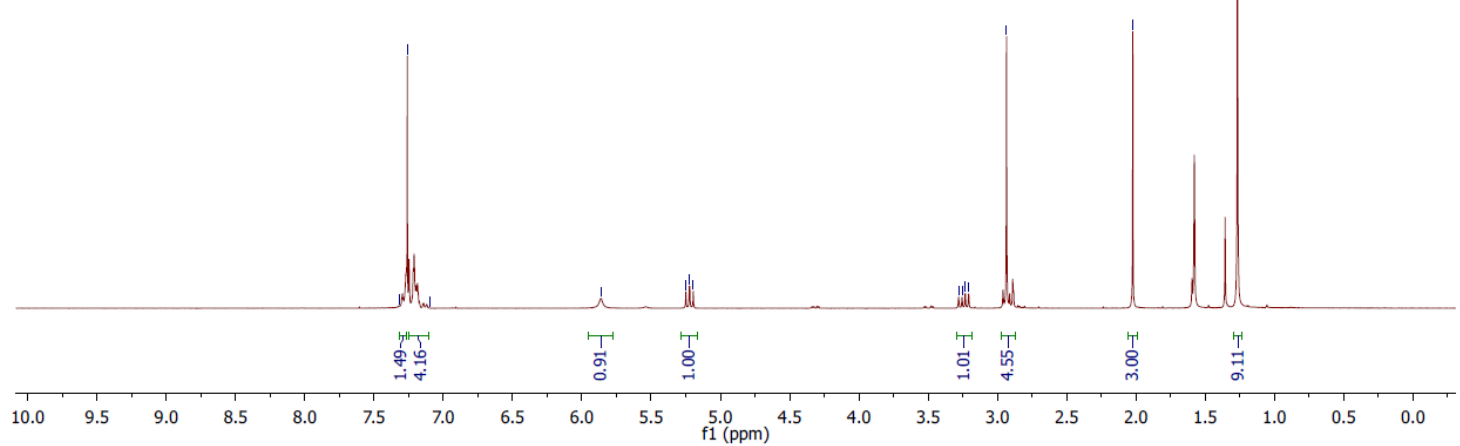

${ }^{13} \mathrm{C}\left\{{ }^{1} \mathrm{H}\right\} \mathrm{NMR}$ of 35 in $\mathrm{CDCl}_{3}$

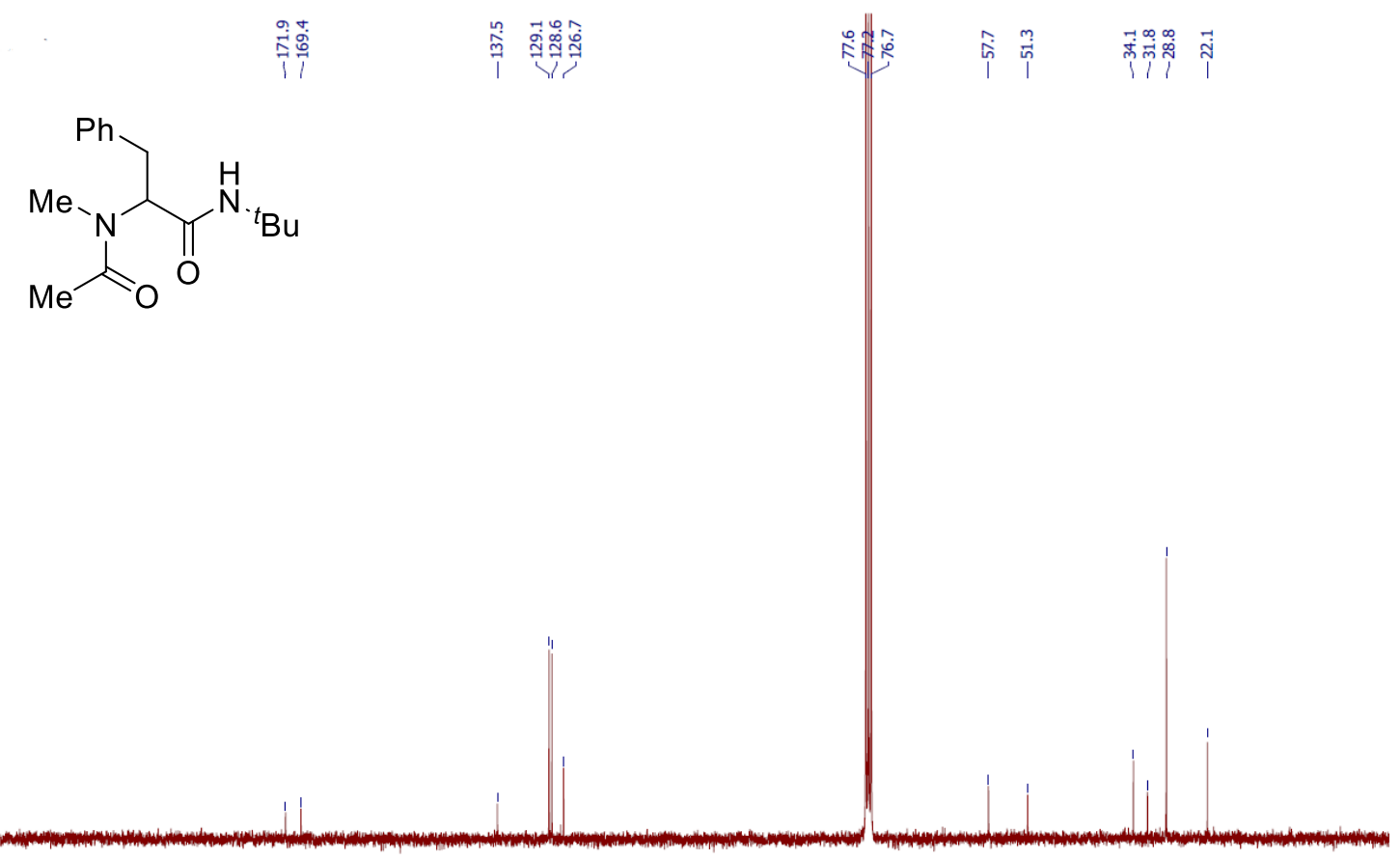

$\begin{array}{llllllllllllllllllllllllll}210 & 200 & 190 & 180 & 170 & 160 & 150 & 140 & 130 & 120 & 110 & 100 & 90 & 80 & 70 & 60 & 50 & 40 & 30 & 20 & 10 & 0\end{array}$ 
${ }^{1} \mathrm{H}$ NMR of 36 in $\mathrm{CDCl}_{3}$

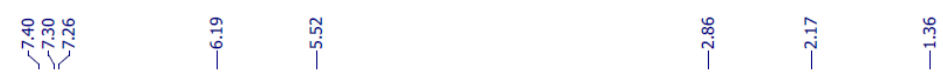<smiles>CC(=O)N(C)C(C(=O)NC(C)(C)C)c1ccccc1</smiles>

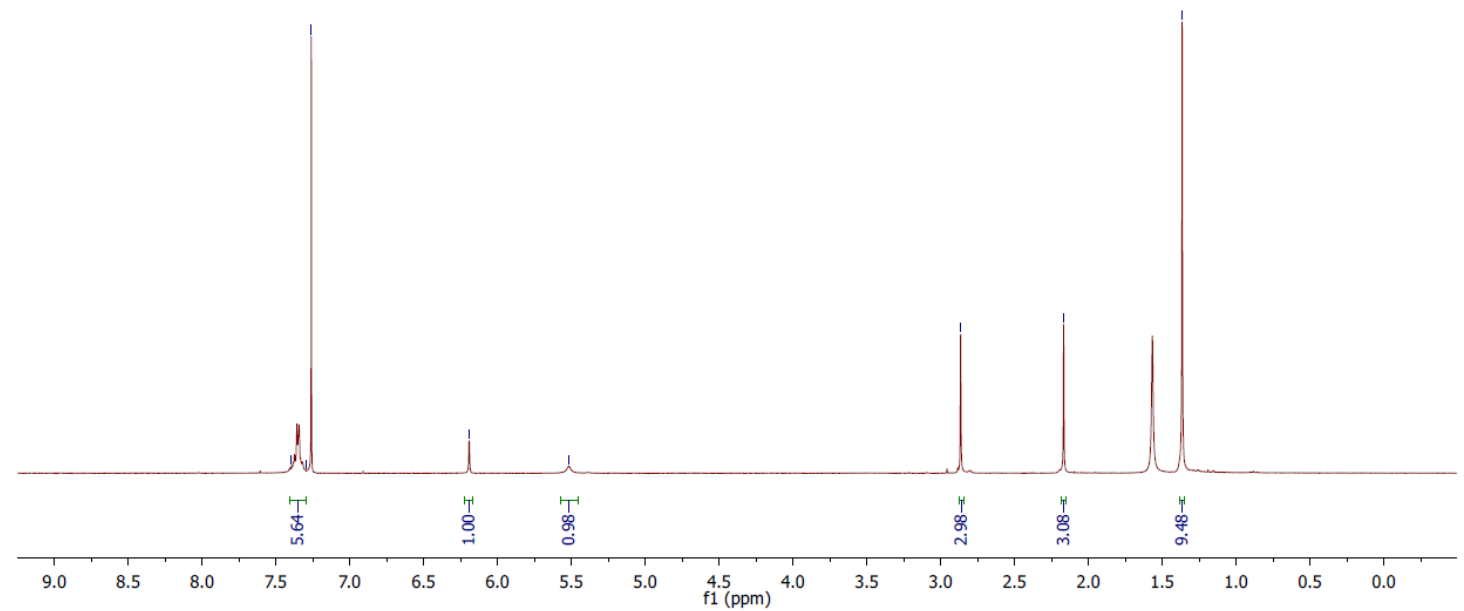

${ }^{13} \mathrm{C}\left\{{ }^{1} \mathrm{H}\right\}$ NMR of 36 in $\mathrm{CDCl}_{3}$

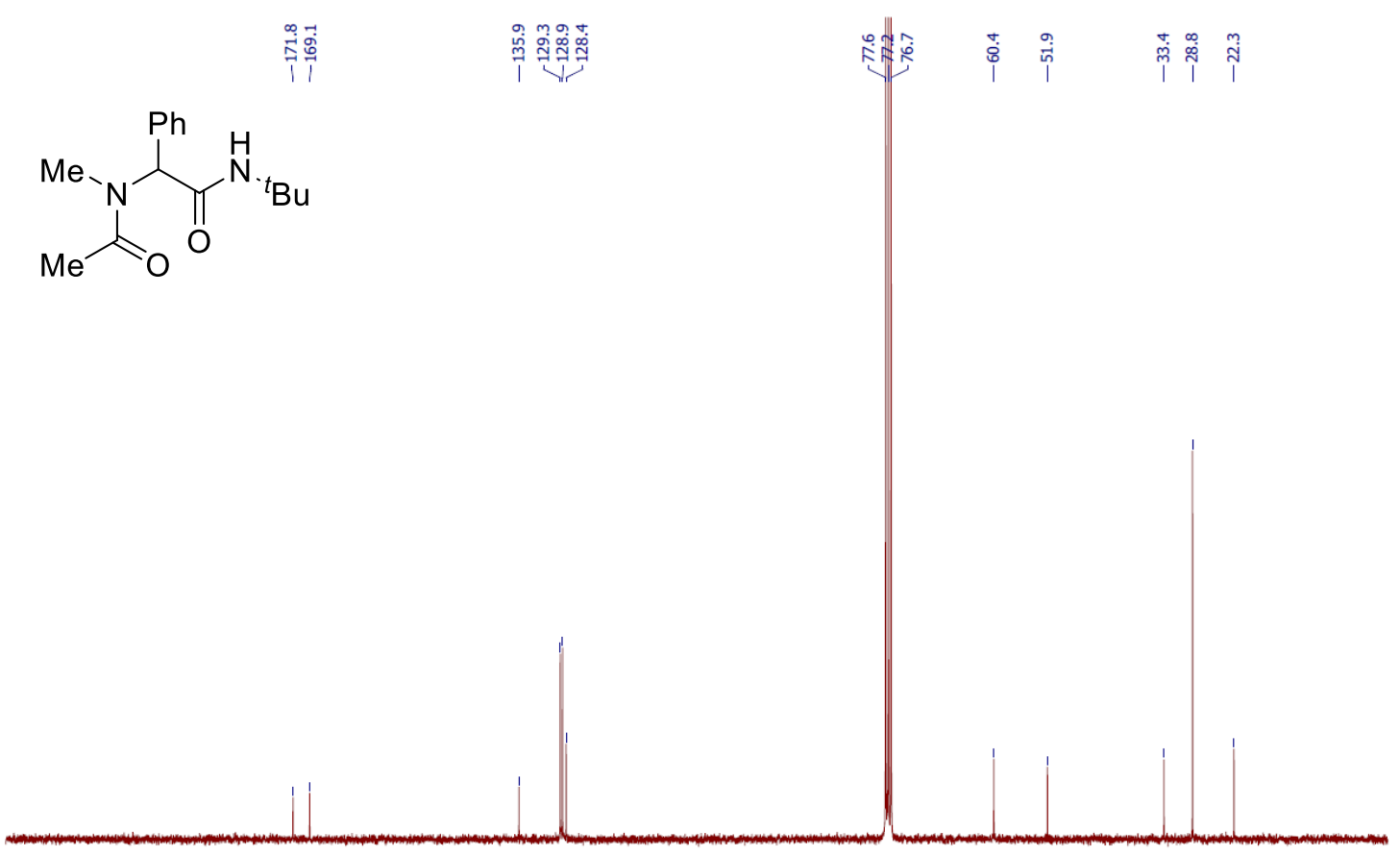

$\begin{array}{llllllllllllllllllllll}210 & 200 & 190 & 180 & 170 & 160 & 150 & 140 & 130 & 120 & \underset{f 1}{110}(\mathrm{ppm}) & 100 & 90 & 80 & 70 & 60 & 50 & 40 & 30 & 20 & 10 & 0\end{array}$ 
${ }^{1} \mathrm{H}$ NMR of 37 in $\mathrm{CDCl}_{3}$

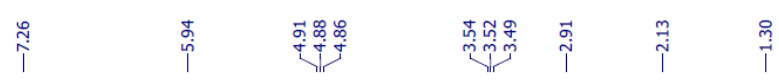<smiles>CC(=O)N(C)C(C[CH]Cl)C(=O)NC(C)(C)C</smiles>

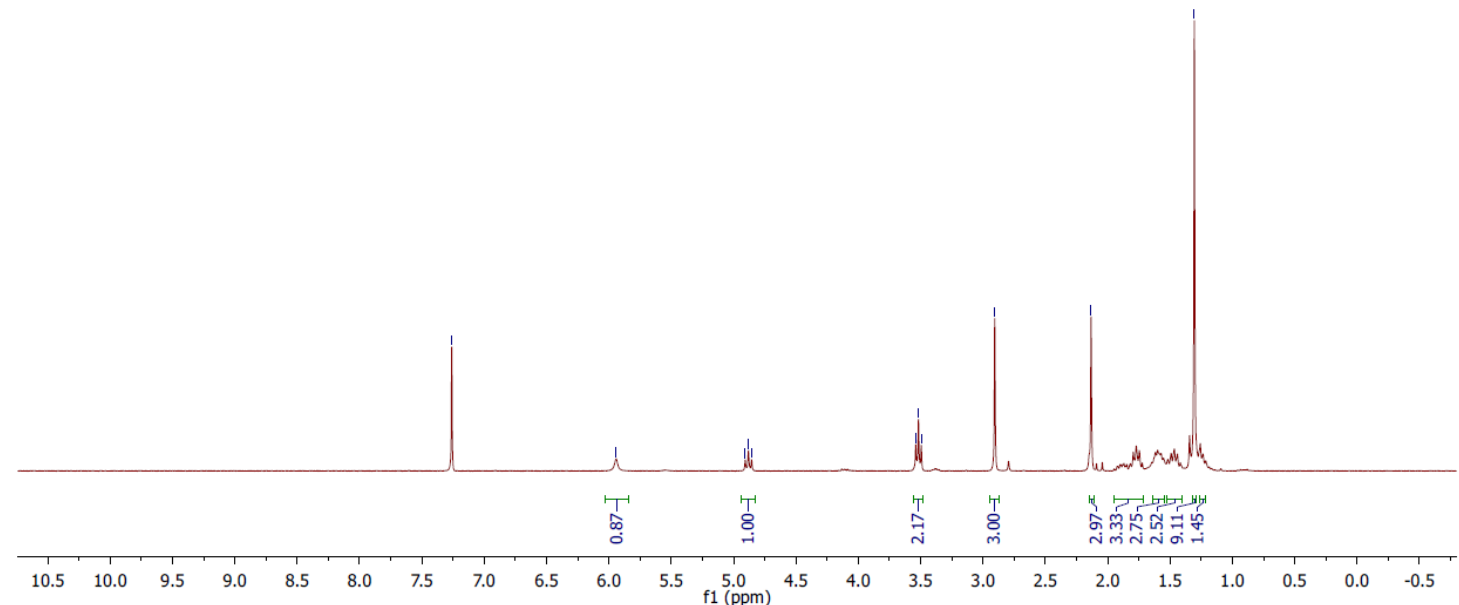

${ }^{13} \mathrm{C}\left\{{ }^{1} \mathrm{H}\right\} \mathrm{NMR}$ of $\mathbf{3 7}$ in $\mathrm{CDCl}_{3}$

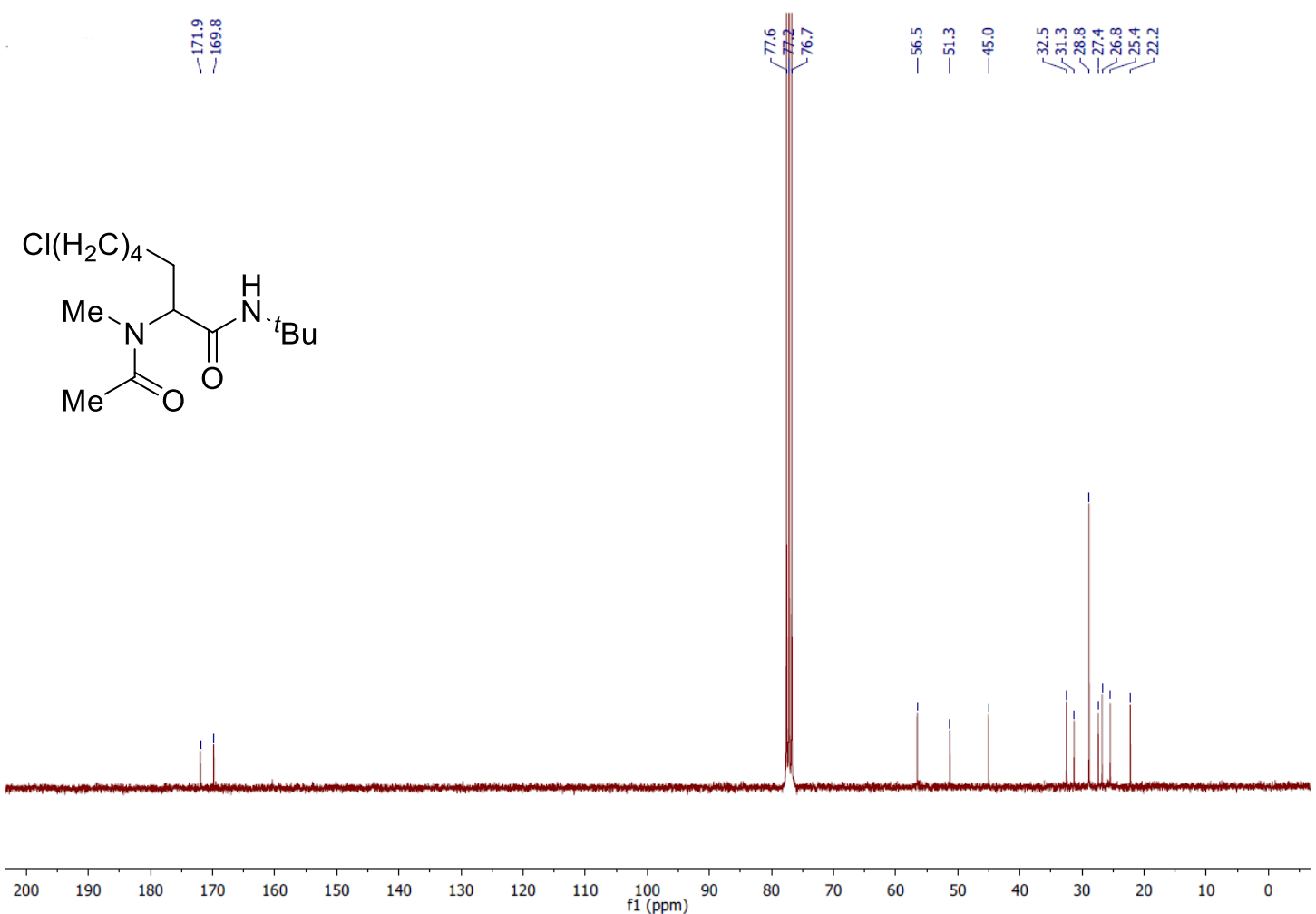


${ }^{1} \mathrm{H}$ NMR of 38 in $\mathrm{CDCl}_{3}$

i<smiles>CC(=O)N(C)C(C[GeH3])C(=O)NC(C)(C)C</smiles>

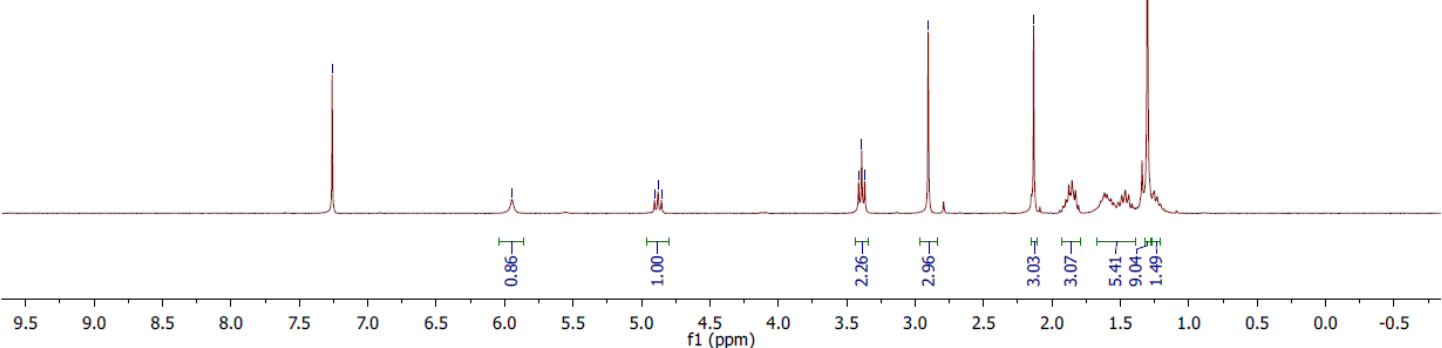

${ }^{13} \mathrm{C}\left\{{ }^{1} \mathrm{H}\right\} \mathrm{NMR}$ of $\mathbf{3 8}$ in $\mathrm{CDCl}_{3}$

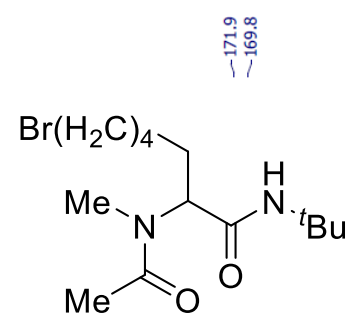

$\begin{array}{lllllllllll}210 & 200 & 190 & 180 & 170 & 160 & 150 & 140 & 130 & 120 & 110 \\ \mathrm{f} 1(\mathrm{ppm})\end{array}$
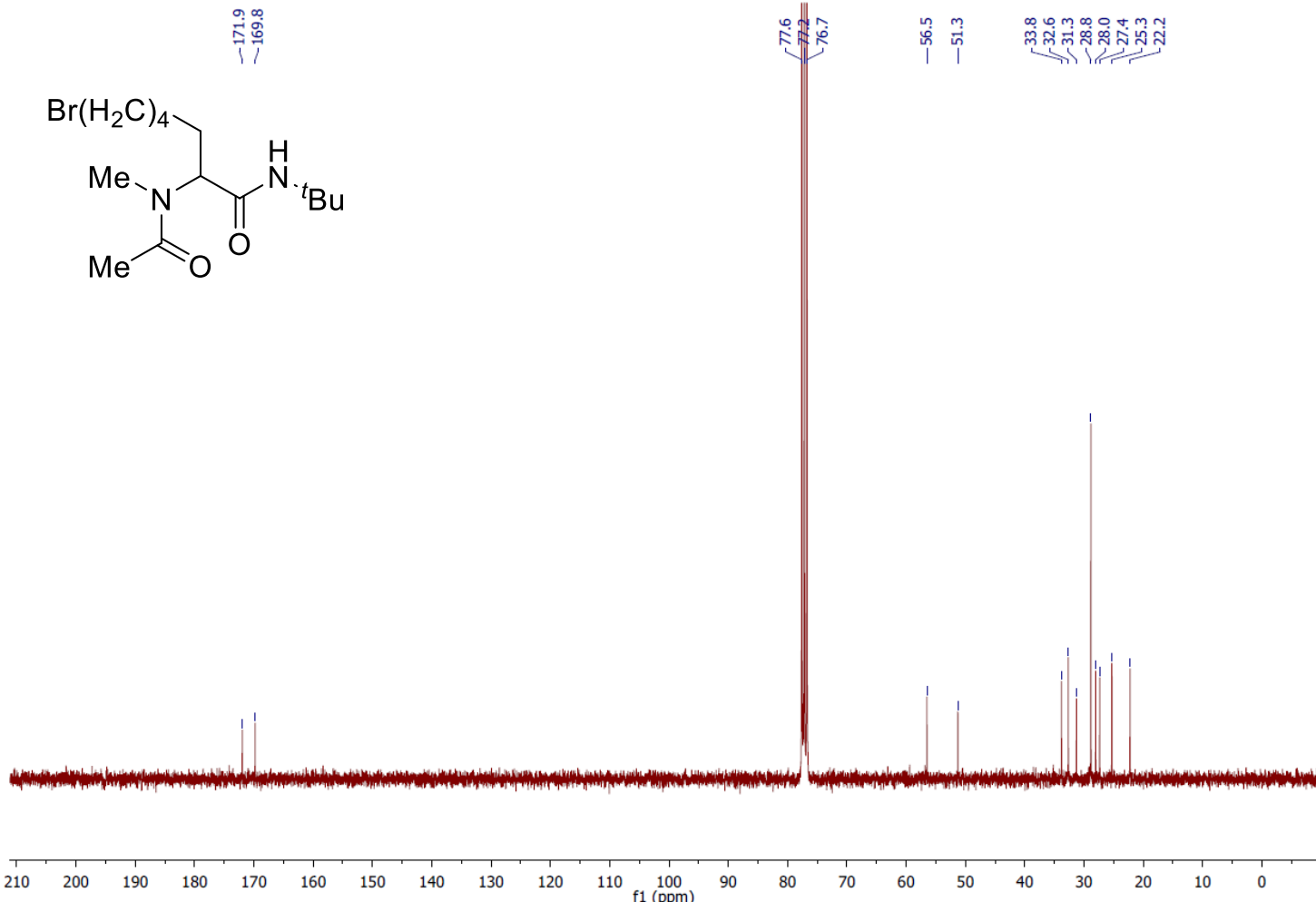
${ }^{1} \mathrm{H}$ NMR of 39 in $\mathrm{CDCl}_{3}$

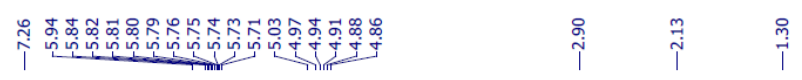<smiles>C=CCCCC(C(=O)NC(C)(C)C)N(C)C(C)=O</smiles>

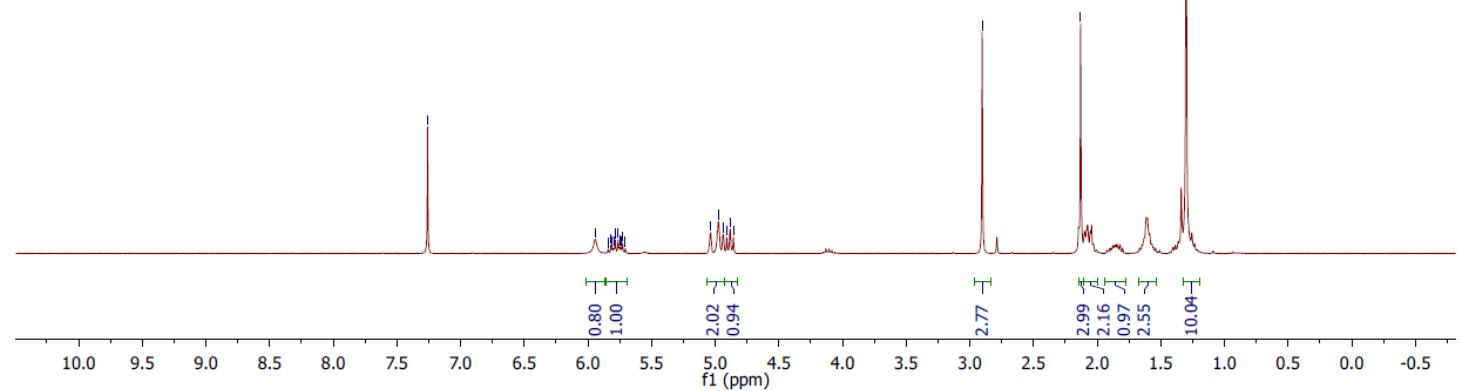

${ }^{13} \mathrm{C}\left\{{ }^{1} \mathrm{H}\right\}$ NMR of 39 in $\mathrm{CDCl}_{3}$

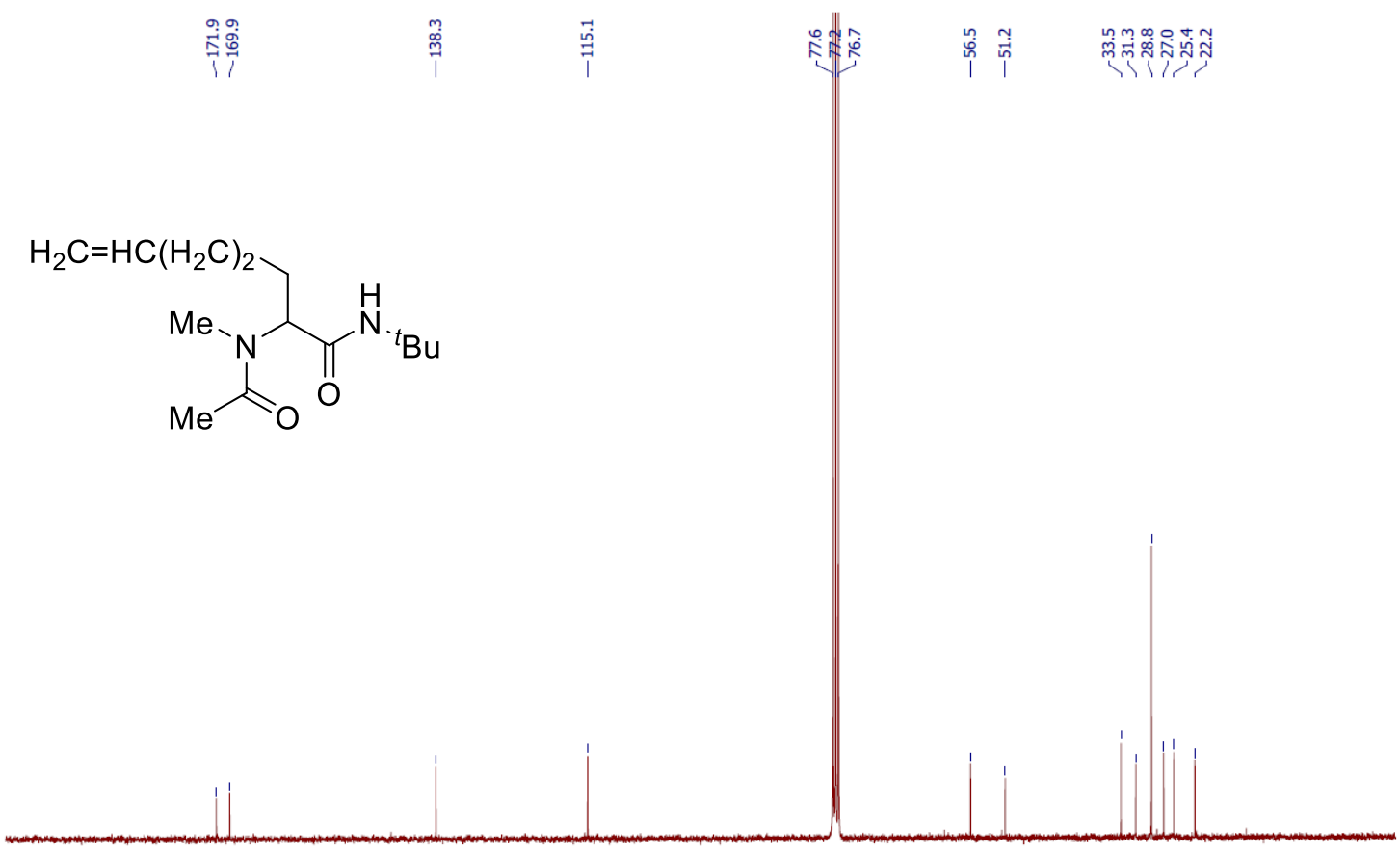

$\begin{array}{lllllllllllllllllllllllllllll} & 190 & 190 & 180 & 170 & 160 & 150 & 140 & 130 & 120 & 110 & 100 & 90 & 80 & 70 & 60 & 50 & 40 & 30 & 20 & 10 & 0\end{array}$ 
${ }^{1} \mathrm{H}$ NMR of 40 in $\mathrm{CDCl}_{3}$

în<smiles>C#CCCCC(C(=O)NC(C)(C)C)N(C)C(C)=O</smiles>

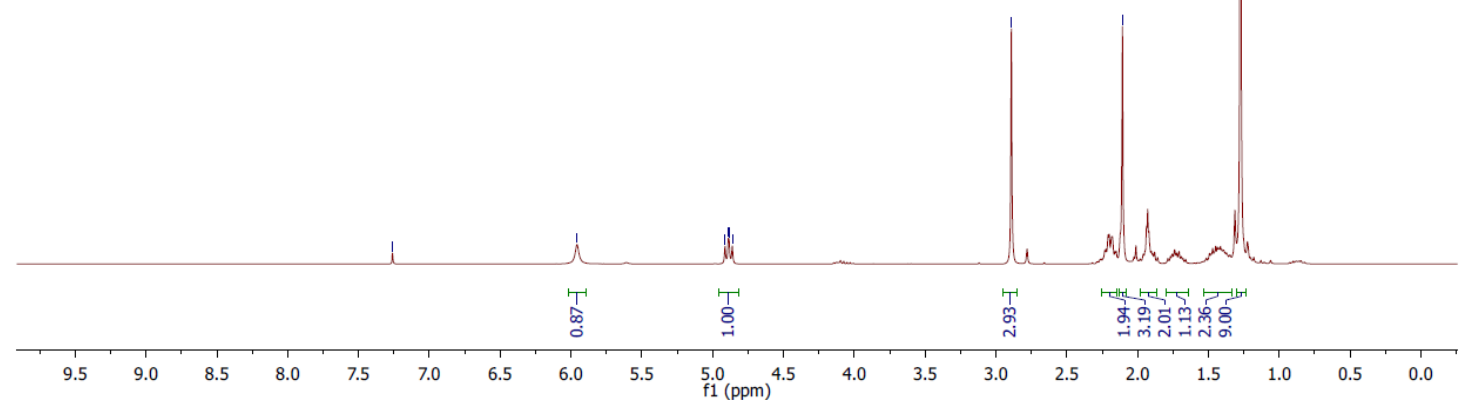

${ }^{13} \mathrm{C}\left\{{ }^{1} \mathrm{H}\right\} \mathrm{NMR}$ of $\mathbf{4 0}$ in $\mathrm{CDCl}_{3}$

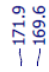

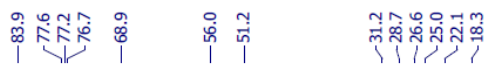<smiles>C#CCCCC(C(=O)NC(C)(C)C)N(C)C(C)=O</smiles>

$200 \quad 190$

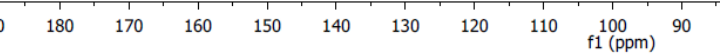


${ }^{1} \mathrm{H}$ NMR of 41 in $\mathrm{CDCl}_{3}$
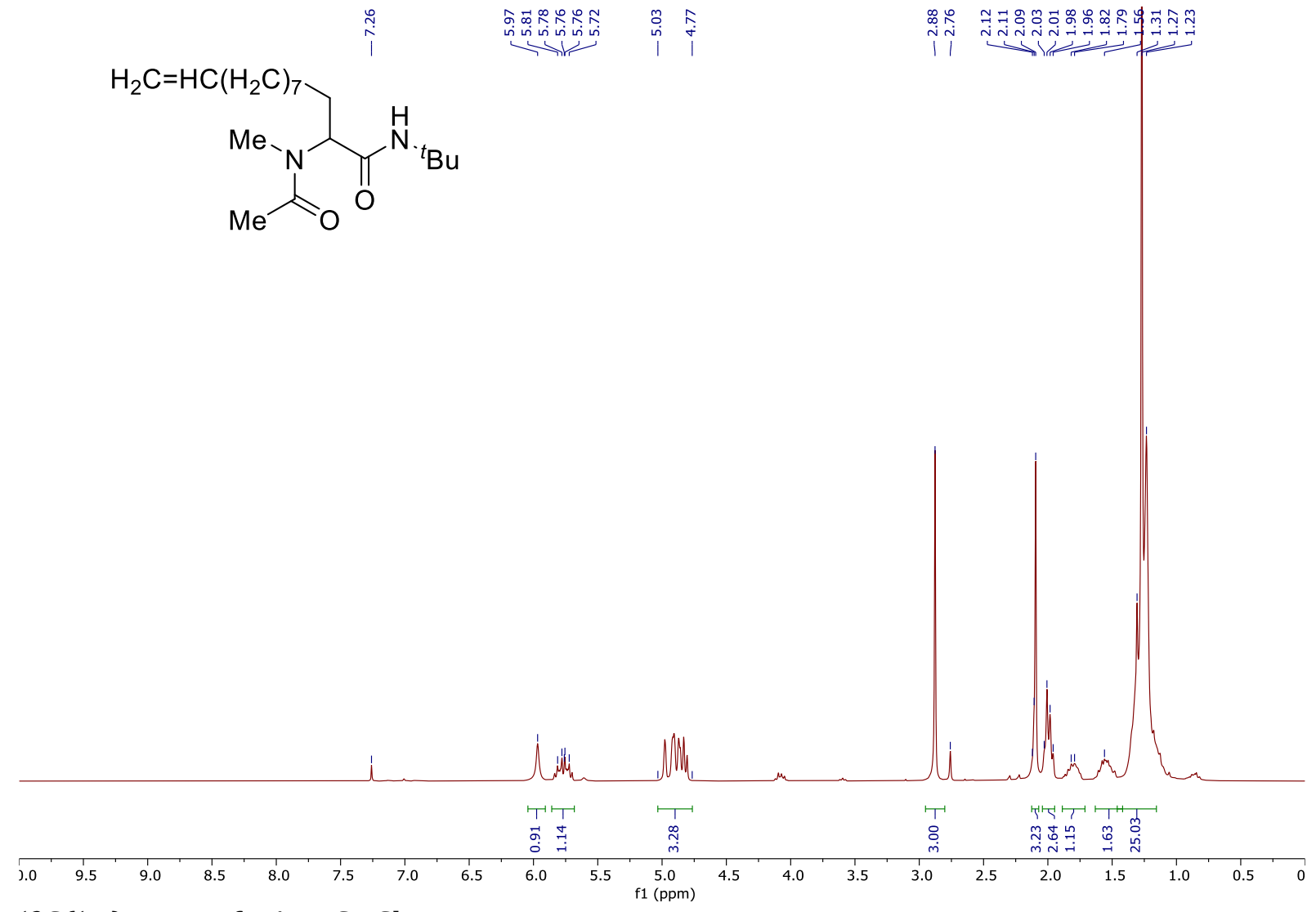

${ }^{13} \mathrm{C}\left\{{ }^{1} \mathrm{H}\right\}$ NMR of 41 in $\mathrm{CDCl}_{3}$
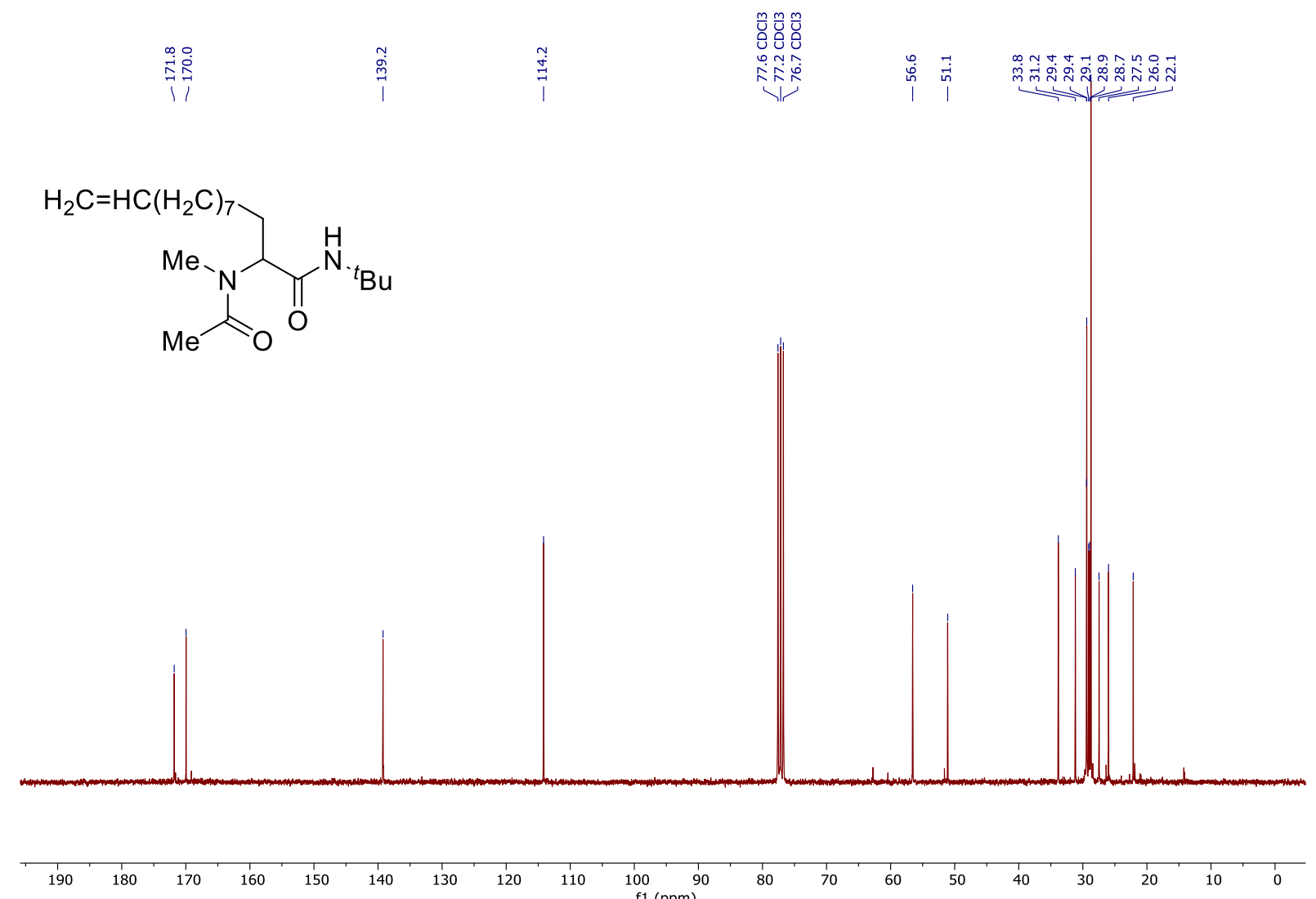